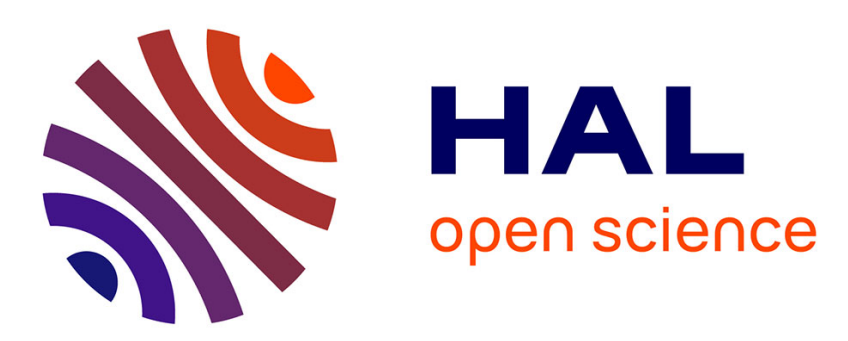

\title{
Investigation of the hyperfine structure of Pr I and Pr II lines based on highly resolved Fourier transform spectra
}

B Gamper, Z Uddin, M Jahangir, O Allard, H Knöckel, E Tiemann, L Windholz

\section{- To cite this version:}

B Gamper, Z Uddin, M Jahangir, O Allard, H Knöckel, et al.. Investigation of the hyperfine structure of Pr I and Pr II lines based on highly resolved Fourier transform spectra. Journal of Physics B: Atomic, Molecular and Optical Physics, 2011, 44 (4), pp.45003. 10.1088/0953-4075/44/4/045003. hal-00596620

\section{HAL Id: hal-00596620 \\ https://hal.science/hal-00596620}

Submitted on 28 May 2011

HAL is a multi-disciplinary open access archive for the deposit and dissemination of scientific research documents, whether they are published or not. The documents may come from teaching and research institutions in France or abroad, or from public or private research centers.
L'archive ouverte pluridisciplinaire HAL, est destinée au dépôt et à la diffusion de documents scientifiques de niveau recherche, publiés ou non, émanant des établissements d'enseignement et de recherche français ou étrangers, des laboratoires publics ou privés. 


\title{
Investigation of the hyperfine structure of Pr I and Pr II lines based on highly resolved Fourier transform spectra
}

\author{
B Gamper ${ }^{1}$, Z Uddin ${ }^{1,2}$, M Jahangir ${ }^{2}$, O Allard $^{1}$, H Knöckel ${ }^{3}$, E \\ Tiemann $^{3}$ and L Windholz ${ }^{1}$ \\ ${ }^{1}$ Institut für Experimentalphysik, Technische Universität Graz, Petersgasse 16, 8010 \\ Graz \\ 2 Department of Physics, University of Karachi, University Road, Karachi 75270 \\ ${ }^{3}$ Institut für Quantenoptik, Leibnitz Universität Hannover, Welfengarten 1, 30167 \\ Hannover \\ E-mail: windholz@tugraz.at
}

\begin{abstract}
We report the recording of new highly resolved Fourier transform spectra of the neutral praseodymium atom. With the help of the new spectra we found about 9000 new lines, from which - in the region 3260 to $9880 \AA$ - 1194 could be classified as transitions between energy levels of the Pr atom and 19 as transitions of the Pr ion. 23 new atomic energy levels of odd parity and one of even parity were discovered during this first examination. The spectra might be very helpful for further laser spectroscopic investigations of the hyperfine structure of Pr I and Pr II transitions.
\end{abstract}




\section{Introduction}

Praseodymium is a metal and belongs to the group of lanthanides. The ${ }^{141} \mathrm{Pr}$ atom has a non zero nuclear spin $I=5 / 2$ due to the odd number of protons $(\mathrm{Z}=59)$. Like other lanthanides also Pr has a very rich and complex optical spectrum; it has a few ten thousands lines from which a big part is not classified. The praseodymium nucleus is almost spherically symmetric, thus it has a very small electric quadrupole moment and the hyperfine constants $B$ are very small. The first studies of the hyperfine (hf) structure of spectral lines of Pr were done by King in 1928 [1]. The next extensive continuation of this work was done by Ginibre in 1988; she presented many new energy levels [2], [3] and [4] and listed approximately 17000 spectral lines. Nevertheless, her list is far from being complete. The Poznań group around Dembczyński performed theoretical interpretation of the hyperfine structure of $\mathrm{Pr}$ and could solve some of the configuration mixing problems [5]. Furmann, also part of the group in Poznan, investigated the hf structure of Pr via the method of laser-induced fluorescence (LIF) spectroscopy since 2005 [6] and [7] and he could enlarge the level list of Pr I and Pr II. Studies of the hf structure of neutral praseodymium have been performed by the group of Guthöhrlein in Hamburg [8] and by our group since 2004 [9, 10]. Even though the enormous work of Ginibre was based on an evaluation of Fourier transform (FT) spectra, the primary spectral data are not available to us. Nevertheless, the line list of Ginibre, containing approximately 17000 lines, was the basis of all laser spectroscopic work until 2007. In these laser spectroscopic experiments, lines which are given by Ginibre but could not be classified by her were excited by laser light, setting the laser wavelength close to the listed wavelength of the center of gravity $(\mathrm{cg})$, and searching for LIF signals.

However, if someone wants to perform LIF spectroscopy more efficiently, a precise setting of the excitation laser frequency is desired. Thus, we decided to take new FT spectra, having a high resolution in order to resolve at least the widely splitted hf patterns. The goal of our investigation is to classify as many lines as possible and to find up to now unknown energy levels in order to increase the knowledge of the stationary states of the electron shells as a basis for a better theoretical description of these states.

\section{Experimental details}

FT spectroscopy has a lot of advantages over other spectroscopic techniques: one can record many thousands lines by one scan under Doppler-limited conditions. With a FT spectrometer it is possible to measure very weak signals and to cover a large wave number range per scan. It has high resolution, large dynamic range of the intensity and a relatively low scan time.

The FT spectra were recorded at the Institute of Quantum Optics at the Leibniz University in Hannover with a continuously scanning high resolution FT-IR spectrometer (IFS $120 \mathrm{HR}$, Bruker Corp.). An example of a sequence of hf structure patterns recorded is shown in figure 1. As a light source we used a home made hollow 
cathode lamp which was the same as we use in Graz for performing laser-induced fluorescence spectroscopy. In the hollow cathode lamp (detailed scheme of the apparatus see [11]) a cloud of free praseodymium atoms is produced by cathode sputtering. The copper cathode is lined with praseodymium and has a typical inner diameter of $3 \mathrm{~mm}$ and a length of $19 \mathrm{~mm}$. The anodes on both sides of the cathode consist of aluminum and are spaced with a ceramic insulator. The discharge was operated with a current of about $65 \mathrm{~mA}(\mathrm{dc})$ using argon as carrier gas at a pressure of about 0.6 mbar. The lamp was cooled by liquid nitrogen to reduce the Doppler width for better hf resolution. The advantage of a hollow cathode lamp is that it produces praseodymium atoms not only in the ground state but also in higher excited states. The emission spectrum of the Pr-Ar plasma under the applied conditions contains mainly Pr I lines, but also Pr II and Ar I and Ar II lines.

The light emitted by the discharge was collected and focused with a set of lenses on the entrance aperture of the FT spectrometer in order to get a Fourier transformed emission spectrum over the very large region from 2380 to $12500 \AA$. As beam splitters we employed the commercial ones provided by the manufacturer: for the near infrared region between $12500 \AA$ and $10000 \AA \mathrm{CaF}_{2}$ coated with silicon, for the visible range from $10000 \AA$ to $4000 \AA$ quartz with dielectric coating and for the UV quartz with $\mathrm{Al}$ coating. The resolution of the spectra is given by the convolution of the instrumental profile and the width of the spectral lines. The entrance aperture was varied from 0.5 to $1.3 \mathrm{~mm}$ diameter. To optimize the signal-to-noise ratio (SNR) the spectra were recorded in parts limiting the recorded ranges by optical dielectric low pass and high pass filters. For the near infrared range a silicon photodiode was used as a detector, while for the visible and UV ranges we employed a photomultiplier. Multiple scans were averaged at a typical resolution of $0.03 \mathrm{~cm}^{-1}$ for an improved SNR.

The original data files of the FT spectrometer contained intensity versus wave number. The specification of the instrument is given to be better than $5 \cdot 10^{-8}$ relative and $5.10^{-7}$ absolute. This is $0.025 \mathrm{~cm}^{-1}$ at $50000 \mathrm{~cm}^{-1}$ or $0.001 \AA$ at $2000 \AA ; 0.0125$ $\mathrm{cm}^{-1}$ at $25000 \mathrm{~cm}^{-1}$ or $0.002 \AA$ at $4000 \AA ; 0.005 \mathrm{~cm}^{-1}$ at $10000 \mathrm{~cm}^{-1}$ or $0.005 \AA$ at $10000 \AA$. Spectra from different regions from IR to UV were combined to form one spectrum. Their correct overlapping was checked by comparing the hf structures of the recorded spectral lines. Then the spectrum was converted to intensity versus air wavelength using the dispersion formula given by Peck and Reader [12]. This spectrum can be handled in graphic form by the existing classification program [13].

\section{Results and discussion}

The amount of spectral lines in Pr is enormous. An example of the FT spectrum in the visible region is given in Figure 1, which shows only a very narrow part of 2.5 $\AA$ of the whole FT spectrum. In this region there are noticeable at least 12 spectral lines with partly resolved hf structure, which are marked by brackets. Meaning of the designations: a: line already classified in ref. [4]; b: mentioned in ref. [4], but not 
classified; c: investigated by laser excitation and classified in ref. [9]; d: new lines, discovered with help of this spectrum. Around $5675.75 \AA$ a typical blend situation can be found: Two of the lines appear to be overlapping. Sometimes even more than two lines with practically the same cg wavelength can be found. This is due to the very big number of electronic energy levels of the Pr atom: Transition frequencies between certain different pairs of levels are nearly or exactly degenerate. For such blend situations the assignment of the correct level pair (this assignment we call "classification") is not possible using only the wave number difference of the levels in comparison with the cg wave number from the FT hf pattern. In addition, the shape of the hf pattern has to be considered. For a huge number of lines the classification is possible since the hf pattern predicted from already known hf constants of the involved levels coincides with the hf pattern observed in the FT spectrum. Of course, this procedure provides that the hf constants of the combining levels are already known from earlier measurements.

In other cases hf constants can be determined using the FT pattern, if the transition is known but the hf constants not. The correctness of the hf constants can be proven by comparing the hf patterns of other lines combining with the level under investigation in the FT spectrum. For this purpose it is necessary to extend the FT spectrum over a range as wide as possible.

If the situation in the observed spectrum is very complex, so that the observed spectral structures can not be clearly distinguished, laser spectroscopic experiments are necessary to divide the excited levels by their characteristic fluorescence lines. In this case the FT spectrum can be used conveniently to set the desired laser excitation wavelength.

All components of the hf patterns of the spectral lines appearing in Figure 1 are also possible excitation wavelengths for laser-induced fluorescence (LIF) spectroscopy. The excitation wavelengths can be determined from FT spectra with an uncertainty of few $0.001 \AA$.

Originally, our database contained approximately 17000 lines of Pr I and Pr II. Up to now we could enlarge this list to about 26000 lines. Many of the 26000 lines are not yet classified, meaning that not every line listed in Ginibres tables [4] or discovered in the present FT spectrum is related to a transition between two already known energy levels. It should be emphasized that our additions to the line list of Ginibre (ref. [4]) are not done automatically by a computer program, but by comparing structures in the graphically depicted FT spectrum with predicted (and graphically represented) hf patterns belonging to transitions which fit in their wave number to the considered part of the FT spectrum. This comparison is possible since in the FT spectrum the relative intensities of the components of an hf pattern fit quite good to the theoretical intensity ratios.

In table 1 all new spectral lines of the praseodymium atom (Pr I) which clearly could be assigned to a transition between two energy levels, are given. This table, containing more than 1200 lines, is available in its full length only in electronic form. In table 2 the Pr I lines of table 1 with an SNR larger than 50, are listed again. Table 
3 gives all the newly classified ionic lines (Pr II), respectively. The classification was performed using the $\mathrm{cg}$ wave numbers and the shape of the hf patterns, resolved well enough to identify the combining levels without any doubts.

All three tables have the same structure: Column 1 contains the cg wavelength in air in $\AA$. Information about the SNR is given in column 2 (per definition, noise is treated to have $\mathrm{SNR}=1$. The $\mathrm{SNR}$ is a guide to the relative intensities of the lines, since the intensities depend strongly on the type of light source and the conditions under which a discharge is operated). If there is written "nl" in front of the SNR it means that this line is a new one, not known before these investigations. All the other lines are mentioned in the list of Ginibre [4] but they are not classified, either because the energy levels involved in the transition were found after she did her work or because the hf patterns were not sufficiently resolved in her spectra. In columns 3 and 5 the J-values of the involved levels are listed. The corresponding energies in $\mathrm{cm}^{-1}$ are given in columns 4 and 6. The energy levels are listed according to their parities, in the column 4 the even ones and in the column 6 the odd ones. If a new level is involved, it is marked with an upper index 'a'.

The cg wavelength for each line given in the tables is obtained by overlapping two graphs: the hf structure obtained from the FT spectrum and a synthetical hf pattern, calculated from the known hf constants of the combining levels. The intensities are normalized for this comparison, and the Gaussian width of the synthetical spectrum has to be chosen close to the width obtained in the FT spectrum. Of course this width is wavelength-dependent. One graph can be shifted against the other in steps of $0.001 \AA$. The correct position gives then the cg wavelength. Since human brain is quite sensitive to a mismatch of two graphs, it is really possible to notice a mismatch of $0.001 \AA$ over the whole wavelength range (3260 to $9880 \AA$ ). Thus, the given wavelengths are good to $0.001 \AA$ for a wide range of SNR. For lines with SNR $<10$ the uncertainty is estimated to be $0.002 \AA$. The absolute wavelength accuracy could be checked in the range 5145 to $3729 \AA$ against a list of Ar-lines used in FT spectrometry for wavelength calibration (see ref. [14]). Agreement was better than $0.001 \AA$. In the UV and IR region Ar-lines of the present spectrum were compared with Ar-lines of earlier used FT spectra of Ta and Ar, taken at Imperial College London and Kitt Peak National Observatory ([15]). Again the agreement was better than $0.003 \AA$. Thus the absolute accuracy of the given wavelengths in our tables is better than $0.003 \AA$.

Pr I energy levels given in Tables 1, 2 (and their hf constants, used for determination of the cg wavelengths) are taken either from the tables of Ginibre [4] or from measurements performed by the group in Hamburg [8] (private communication). The wave numbers have been fine adapted by means of the present FT spectrum. Under assumption that the energy of the lowest even state, $4432.24 \mathrm{~cm}^{-1}$, as given in ref. [16], is correct, we have first energy corrected high lying odd levels, combining with this lower level. Then we have used transitions from these levels to the second lower even energy state to correct its wave number. From this level we were going up to other odd levels and so on. The same procedure was done beginning with the odd ground state level. 
The differences of the here given level energies fit to the wave numbers, calculated from the given wavelengths, better than $0.01 \mathrm{~cm}^{-1}$. Energy values given in Table 3 are from Ginibre [3].

For some well resolved lines, which could not be classified as transitions between known levels, it was possible to determine the hf constants and to identify one of the involved levels by its hf constants. Then a new level could be introduced combining the wave number of the identified level and the wave number of the transition. The proof that such a level exists is done by calculating all possible decay wavelengths and looking into the corresponding regions of the FT spectrum for the predicted pattern. If observed structures fit with the expected pattern and the expected cg wavelength, the level is assumed to be really existing. This method of finding new levels is especially important for spectral regions where laser excitation is difficult, e.g. in the IR or UV.

In this way, we could find several new energy levels. The hf constants were determined by fitting the hf pattern of the line under investigation, using the program "Fitter" ([17]). These levels are marked in table 1 and table 2 with subscript "a". The results are shown in table 4 . Column 1 of this table gives the energies in $\mathrm{cm}^{-1}$ of the newly discovered levels; the accuracy depends mainly on the SNR of the investigated hf patterns. Their J-values are listed in column 2; in the next column the hyperfine constants A (in $\mathrm{MHz}$ ) are given. Due to the limited resolution of the FT spectrum the value of $\mathrm{B}$ was assumed to be zero. The $5^{\text {th }}$ column tells us which lines (wavelengths in air in $\AA$ ) can be explained with the new levels. The corresponding transitions can be found in table 1.

As an example we discuss the line with the cg wavelength $9319.006 \AA$. The relative intensities and distribution of the components (Figure 2) suggest a transition with $\Delta J=-1$ with almost equal values of the hyperfine constants $A$ of both levels. The small sharp peak at the high frequency side of this hf structure pattern is generated under this hypothesis by the coincidence of the five off diagonal $(\Delta F=0)$ hyperfine components within the Doppler width. We fitted the structure assuming $J=17 / 2$ and $J^{\prime}=15 / 2$ (the prime marks the upper level of the transition), and got $A=970 \mathrm{MHz}$ and $A^{\prime}=961 \mathrm{MHz}$. Then we looked in our level data base for a level with $J$ and $A$ as determined in the fitting process. We found an even level with the energy 11714.367 $\mathrm{cm}^{-1}$, with $A=970.2(3) \mathrm{MHz}, B=-16(4) \mathrm{MHz}$ and $J=17 / 2$. Assuming that this level is the lower level involved in this transition, we fitted the structure again with fixed hyperfine constants of the lower level and added the $\mathrm{cg}$ wave number of the investigated line to the wave number of the lower level. Finally we determined the new level to have 22442.185(5) $\mathrm{cm}^{-1}, J=15 / 2, A^{\prime}=959(9) \mathrm{MHz}$ with odd parity. Fig. 3 shows the hf structure calculated with J, A and J', A' in comparison with the pattern in the FT spectrum. The new level explains 5 other lines in the IR region and 3 lines in the visible region (see Table 4 ).

We have to emphasize that such a fit is only possible if some of the small components (or a convolution of them) are clearly resolved in the FT spectra. Otherwise, the splitting of the diagonal components is more sensitive to the difference $\left(A-A^{\prime}\right)$ than to the 
absolute values of $A, A^{\prime}$, and one cannot identify one of the involved levels by its Avalue.

Altogether 24 new levels could be found by the analysis of FT patterns (mainly located in the IR region); each level explains other previously unclassified lines.

Table 1: Spectral lines of Pr I which clearly could be assigned to a transition between two energy levels

\begin{tabular}{|c|c|c|c|c|c|}
\hline \multirow[b]{2}{*}{$\lambda / \AA$} & \multirow[b]{2}{*}{ SNR } & \multicolumn{2}{|r|}{ Even } & \multicolumn{2}{|r|}{ Odd } \\
\hline & & J-value & Level Energy $/ \mathrm{cm}^{-1}$ & J-value & Level Energy $/ \mathrm{cm}^{-1}$ \\
\hline 3264.301 & nl 15 & $9 / 2$ & 30625.599 & $9 / 2$ & 0 \\
\hline 3308.390 & nl 4 & $11 / 2$ & 30217.535 & $9 / 2$ & 0 \\
\hline 3363.938 & nl 53 & $11 / 2$ & 29718.524 & $9 / 2$ & 0 \\
\hline 3437.597 & nl 36 & $13 / 2$ & 30458.359 & $11 / 2$ & 1376.605 \\
\hline 3482.081 & $\mathrm{nl} 7$ & $11 / 2$ & 30086.879 & $11 / 2$ & 1376.605 \\
\hline 3500.325 & nl 20 & $11 / 2$ & 28560.604 & $9 / 2$ & 0 \\
\hline 3518.513 & nl 16 & $7 / 2$ & 28412.960 & $9 / 2$ & 0 \\
\hline 3551.415 & nl 9 & $11 / 2$ & 28149.750 & $9 / 2$ & 0 \\
\hline 3617.638 & $\mathrm{nl} 13$ & $11 / 2$ & 27634.464 & $9 / 2$ & 0 \\
\hline 3650.240 & nl 7 & $11 / 2$ & 28764.269 & $11 / 2$ & 1376.605 \\
\hline 3654.616 & $\mathrm{nl} 44$ & $9 / 2$ & 4432.240 & $11 / 2$ & 31787.102 \\
\hline 3670.398 & nl 7 & $9 / 2$ & 8029.290 & $11 / 2$ & 35266.525 \\
\hline 3676.089 & $\mathrm{nl} 6$ & $11 / 2$ & 27195.110 & $9 / 2$ & 0 \\
\hline 3681.669 & $\mathrm{nl} 26$ & $11 / 2$ & 27153.870 & $9 / 2$ & 0 \\
\hline 3693.608 & $\mathrm{nl} 15$ & $11 / 2$ & 27066.095 & $9 / 2$ & 0 \\
\hline 3728.799 & $\mathrm{nl} 5$ & $9 / 2$ & 9105.035 & $11 / 2$ & 35915.701 \\
\hline 3732.473 & nl 48 & $11 / 2$ & 26784.270 & $9 / 2$ & 0 \\
\hline 3734.024 & nl 24 & $11 / 2$ & 28149.750 & $11 / 2$ & 1376.605 \\
\hline 3738.704 & nl 7 & $11 / 2$ & 6313.239 & $13 / 2$ & 33052.863 \\
\hline 3744.240 & nl 8 & $15 / 2$ & 9745.420 & $17 / 2$ & 36445.505 \\
\hline 3751.945 & $\mathrm{nl} 4$ & $9 / 2$ & 4432.240 & $11 / 2$ & 31077.525 \\
\hline 3764.900 & $\mathrm{nl} 8$ & $9 / 2$ & 4432.240 & $9 / 2$ & 30985.825 \\
\hline 3782.853 & nl 8 & $9 / 2$ & 4432.240 & $11 / 2$ & 30859.807 \\
\hline 3785.058 & $\mathrm{nl} 16$ & $11 / 2$ & 4866.530 & $13 / 2$ & 31278.701 \\
\hline 3789.689 & nl 17 & $7 / 2$ & 6535.587 & $9 / 2$ & 32915.484 \\
\hline 3789.738 & $\mathrm{nl} 10$ & $13 / 2$ & 6603.606 & $15 / 2$ & 32983.162 \\
\hline 3792.514 & 14 & $11 / 2$ & 4866.530 & $13 / 2$ & 31226.830 \\
\hline 3792.953 & nl 10 & $11 / 2$ & 26357.240 & $9 / 2$ & 0 \\
\hline 3796.162 & nl 8 & $11 / 2$ & 4866.530 & $13 / 2$ & 31201.444 \\
\hline 3822.768 & nl 13 & $9 / 2$ & 30583.869 & $11 / 2$ & 4432.240 \\
\hline 3832.285 & nl 14 & $11 / 2$ & 4866.530 & $13 / 2$ & 30953.221 \\
\hline 3836.285 & nl 12 & $15 / 2$ & 28906.235 & $13 / 2$ & 2846.744 \\
\hline 3844.099 & nl 11 & $11 / 2$ & 6313.239 & $13 / 2$ & 32319.759 \\
\hline 3845.830 & nl 9 & $15 / 2$ & 8363.910 & $17 / 2$ & 34358.775 \\
\hline 3850.364 & $\mathrm{nl} 23$ & $9 / 2$ & 5822.905 & $11 / 2$ & 31787.102 \\
\hline 3895.616 & nl 20 & $9 / 2$ & 4432.240 & $11 / 2$ & 30094.849 \\
\hline 3920.839 & nl 5 & $9 / 2$ & 4432.240 & $9 / 2$ & 29929.763 \\
\hline 3955.220 & nl 12 & $11 / 2$ & 4866.530 & $13 / 2$ & 30142.420 \\
\hline 3966.271 & nl 19 & $9 / 2$ & 25205.463 & $9 / 2$ & 0 \\
\hline
\end{tabular}

$\mathrm{nl}$... new line, ${ }^{a}$ previously unknown energy level 
Table 1 - Continued

\begin{tabular}{|c|c|c|c|c|c|}
\hline \multirow[b]{2}{*}{$\lambda / \AA$} & \multirow[b]{2}{*}{ SNR } & \multicolumn{2}{|r|}{ Even } & \multicolumn{2}{|r|}{ Odd } \\
\hline & & J-value & Level Energy $/ \mathrm{cm}^{-1}$ & J-value & Level Energy $/ \mathrm{cm}^{-1}$ \\
\hline 3977.315 & nl 12 & $11 / 2$ & 25135.518 & $9 / 2$ & 0 \\
\hline 3983.367 & 17 & $11 / 2$ & 4866.530 & $9 / 2$ & 29963.821 \\
\hline 3983.837 & nl 14 & $9 / 2$ & 4432.240 & $11 / 2$ & 29526.571 \\
\hline 3994.341 & nl 20 & $11 / 2$ & 4866.530 & $13 / 2$ & 29894.870 \\
\hline 4010.870 & nl 6 & $9 / 2$ & 4432.240 & $11 / 2$ & 29357.439 \\
\hline 4018.223 & nl 5 & $11 / 2$ & 4866.530 & $13 / 2$ & 29746.124 \\
\hline 4064.250 & nl 9 & $13 / 2$ & 6603.606 & $13 / 2$ & 31201.444 \\
\hline 4067.606 & nl 6 & $11 / 2$ & 4866.530 & $13 / 2$ & 29444.080 \\
\hline 4082.316 & nl 11 & $11 / 2$ & 4866.530 & $9 / 2$ & 29355.515 \\
\hline 4095.244 & nl 8 & $9 / 2$ & 4432.240 & $7 / 2$ & 28843.919 \\
\hline 4099.596 & nl 5 & $11 / 2$ & 6892.950 & $13 / 2$ & 31278.760 \\
\hline 4116.357 & nl 19 & $15 / 2$ & 27133.216 & $13 / 2$ & 2846.744 \\
\hline 4140.536 & nl 8 & $13 / 2$ & 6603.630 & $11 / 2$ & 30748.179 \\
\hline 4146.146 & nl 8 & $9 / 2$ & 24111.970 & $9 / 2$ & 0 \\
\hline 4158.484 & nl 9 & $9 / 2$ & 5822.905 & $9 / 2$ & 29863.351 \\
\hline 4181.612 & nl 11 & $9 / 2$ & 4432.240 & $7 / 2$ & 28339.724 \\
\hline 4205.089 & nl 6 & $9 / 2$ & 5822.905 & $11 / 2$ & 29596.942 \\
\hline 4214.208 & nl 29 & $11 / 2$ & 6313.239 & $9 / 2$ & 30035.807 \\
\hline 4216.768 & nl 53 & $11 / 2$ & 4866.530 & $13 / 2$ & 28574.696 \\
\hline 4219.806 & nl 10 & $9 / 2$ & 4432.240 & $7 / 2$ & 28123.338 \\
\hline 4223.198 & nl 16 & $9 / 2$ & 4432.240 & $7 / 2$ & 28104.309 \\
\hline 4232.307 & $\mathrm{nl} 10$ & $11 / 2$ & 4866.530 & $13 / 2$ & 28487.651 \\
\hline 4234.681 & $\mathrm{nl} 5$ & $13 / 2$ & 6603.606 & $11 / 2$ & 30211.500 \\
\hline 4236.994 & nl 20 & $9 / 2$ & 4432.240 & $7 / 2$ & 28027.233 \\
\hline 4242.580 & 6 & $11 / 2$ & 4866.530 & $11 / 2$ & 28430.480 \\
\hline 4242.853 & nl 6 & $11 / 2$ & 6892.950 & $13 / 2$ & 30455.358 \\
\hline 4247.880 & $\mathrm{nl} 4$ & $9 / 2$ & 5822.890 & $11 / 2$ & 29357.427 \\
\hline 4252.039 & nl 8 & $9 / 2$ & 4432.240 & $7 / 2$ & 27943.748 \\
\hline 4252.622 & nl 15 & $11 / 2$ & 4866.530 & $13 / 2$ & 28374.815 \\
\hline 4253.281 & nl 13 & $11 / 2$ & 6313.239 & $9 / 2$ & 29817.885 \\
\hline 4257.426 & nl 6 & $9 / 2$ & 4432.240 & $9 / 2$ & 27913.992 \\
\hline 4265.477 & nl 9 & $11 / 2$ & 4866.530 & $13 / 2$ & 28303.969 \\
\hline 4270.230 & nl 16 & $11 / 2$ & 6892.949 & $9 / 2$ & 30304.302 \\
\hline 4273.079 & nl 9 & $13 / 2$ & 6603.606 & $13 / 2$ & 29999.348 \\
\hline 4277.584 & nl 7 & $9 / 2$ & 4432.240 & $7 / 2$ & 27803.401 \\
\hline 4287.225 & nl 5 & $11 / 2$ & 6892.949 & $11 / 2$ & 30211.500 \\
\hline 4290.514 & nl 14 & $9 / 2$ & 24677.280 & $11 / 2$ & 1376.605 \\
\hline 4307.900 & $\mathrm{nl} 6$ & $9 / 2$ & 5822.905 & $11 / 2$ & 29029.544 \\
\hline 4315.405 & nl 24 & $9 / 2$ & 5822.905 & $7 / 2$ & 28989.185 \\
\hline 4319.208 & nl 7 & $7 / 2$ & 7617.455 & $5 / 2$ & 30763.338 \\
\hline 4325.278 & nl 16 & $5 / 2$ & 6451.823 & $3 / 2$ & 29565.225 \\
\hline 4325.719 & nl 28 & $11 / 2$ & 6313.239 & $9 / 2$ & 29424.282 \\
\hline 4328.899 & nl 30 & $9 / 2$ & 4432.240 & $7 / 2$ & 27526.308 \\
\hline 4331.816 & nl 6 & $11 / 2$ & 4866.530 & $9 / 2$ & 27945.047 \\
\hline 4337.942 & nl 11 & $9 / 2$ & 5822.905 & $7 / 2$ & 28868.744 \\
\hline 4356.485 & nl 6 & $9 / 2$ & 4432.240 & $11 / 2$ & 27380.075 \\
\hline 4358.728 & nl 9 & $9 / 2$ & 4432.240 & $9 / 2$ & 27368.267 \\
\hline
\end{tabular}

$\mathrm{nl}$... new line, ${ }^{a}$ previously unknown energy level 
Table 1 - Continued

\begin{tabular}{|c|c|c|c|c|c|}
\hline \multirow[b]{2}{*}{$\lambda / \AA$} & \multirow[b]{2}{*}{ SNR } & \multicolumn{2}{|r|}{ Even } & \multicolumn{2}{|r|}{ Odd } \\
\hline & & J-value & Level Energy $/ \mathrm{cm}^{-1}$ & J-value & Level Energy $/ \mathrm{cm}^{-1}$ \\
\hline 4363.971 & nl 15 & $13 / 2$ & 7951.338 & $11 / 2$ & 30859.807 \\
\hline 4364.944 & nl 6 & $9 / 2$ & 12519.718 & $9 / 2$ & 35422.868 \\
\hline 4392.068 & nl 9 & $9 / 2$ & 22761.920 & $9 / 2$ & 0 \\
\hline 4397.193 & nl 29 & $9 / 2$ & 24112.000 & $11 / 2$ & 1376.605 \\
\hline 4403.569 & nl 9 & $9 / 2$ & 5822.890 & $7 / 2$ & 28525.354 \\
\hline 4410.844 & nl 13 & $9 / 2$ & 4432.240 & $11 / 2$ & 27097.270 \\
\hline 4415.835 & $\mathrm{nl} 5$ & $11 / 2$ & 9675.040 & $13 / 2$ & 32314.433 \\
\hline 4417.178 & nl 27 & $13 / 2$ & 7951.338 & $11 / 2$ & 30583.869 \\
\hline 4420.916 & nl 9 & $7 / 2$ & 6535.530 & $9 / 2$ & 29149.238 \\
\hline 4422.438 & nl 11 & $9 / 2$ & 8029.290 & $9 / 2$ & 30634.880 \\
\hline 4424.660 & nl 9 & $9 / 2$ & 4432.240 & $9 / 2$ & 27026.500 \\
\hline 4425.857 & nl 10 & $9 / 2$ & 8029.290 & $11 / 2$ & 30617.441 \\
\hline 4428.933 & nl 6 & $11 / 2$ & 4866.530 & $9 / 2$ & 27438.992 \\
\hline 4431.893 & nl 9 & $11 / 2$ & 4866.530 & $13 / 2$ & 27423.911 \\
\hline 4435.831 & nl 12 & $5 / 2$ & 6451.823 & $7 / 2$ & 28989.185 \\
\hline 4436.837 & nl 6 & $9 / 2$ & 4432.240 & $11 / 2$ & 26964.492 \\
\hline 4437.451 & nl 23 & $11 / 2$ & 22529.130 & $9 / 2$ & 0 \\
\hline 4438.021 & nl 5 & $9 / 2$ & 4432.240 & $11 / 2$ & 26958.490 \\
\hline 4439.380 & $\mathrm{nl} 4$ & $7 / 2$ & 7617.440 & $7 / 2$ & 30136.763 \\
\hline 4439.614 & nl 7 & $9 / 2$ & 8029.290 & $7 / 2$ & 30547.449 \\
\hline 4458.426 & nl 12 & $13 / 2$ & 7630.170 & $11 / 2$ & 30053.346 \\
\hline 4461.920 & nl 12 & $9 / 2$ & 4432.240 & $9 / 2$ & 26837.828 \\
\hline 4462.201 & nl 11 & $11 / 2$ & 6892.949 & $9 / 2$ & 29297.126 \\
\hline 4462.594 & nl 6 & $9 / 2$ & 8029.290 & $7 / 2$ & 30431.492 \\
\hline 4466.734 & nl 9 & $9 / 2$ & 5822.905 & $7 / 2$ & 28204.346 \\
\hline 4468.215 & nl 26 & $15 / 2$ & 9745.420 & $13 / 2$ & 32119.424 \\
\hline 4482.960 & nl 19 & $9 / 2$ & 5822.905 & $7 / 2$ & 28123.338 \\
\hline 4487.041 & nl 13 & $5 / 2$ & 6451.823 & $5 / 2$ & 28731.850 \\
\hline 4487.412 & nl 7 & $9 / 2$ & 4432.240 & $11 / 2$ & 26710.548 \\
\hline 4492.176 & nl 25 & $13 / 2$ & 7951.338 & $15 / 2$ & 30206.021 \\
\hline 4494.133 & nl 10 & $9 / 2$ & 9105.035 & $9 / 2$ & 31350.008 \\
\hline 4497.014 & nl 11 & $11 / 2$ & 4866.530 & $11 / 2$ & 27097.270 \\
\hline 4502.013 & nl 19 & $13 / 2$ & 6603.606 & $11 / 2$ & 28809.661 \\
\hline 4502.814 & 10 & $9 / 2$ & 4432.240 & $9 / 2$ & 26634.346 \\
\hline 4508.438 & nl 12 & $11 / 2$ & 6313.239 & $13 / 2$ & 28487.651 \\
\hline 4512.158 & nl 13 & $9 / 2$ & 4432.240 & $11 / 2$ & 26588.368 \\
\hline 4519.626 & nl 112 & $15 / 2$ & 8363.916 & $15 / 2$ & 30483.514 \\
\hline 4522.684 & nl 5 & $9 / 2$ & 4432.240 & $11 / 2$ & 26536.814 \\
\hline 4523.816 & nl 14 & $11 / 2$ & 6313.250 & $11 / 2$ & 28412.285 \\
\hline 4523.989 & nl 19 & $9 / 2$ & 5822.890 & $7 / 2$ & 27921.182 \\
\hline 4531.497 & $\mathrm{nl} 30$ & $11 / 2$ & 6313.239 & $13 / 2$ & 28374.815 \\
\hline 4533.924 & nl 25 & $9 / 2$ & 4432.240 & $9 / 2$ & 26482.007 \\
\hline 4534.285 & nl 15 & $13 / 2$ & 7951.338 & $13 / 2$ & 29999.348 \\
\hline 4534.348 & nl 5 & $9 / 2$ & 4432.240 & $11 / 2$ & 26479.944 \\
\hline 4534.881 & nl 8 & $11 / 2$ & 6892.949 & $11 / 2$ & 28938.062 \\
\hline 4541.35 & nl 5 & $11 / 2$ & 11282.870 & $13 / 2$ & 33296.230 \\
\hline 4546.096 & nl 6 & $11 / 2$ & 6313.239 & $13 / 2$ & 28303.969 \\
\hline
\end{tabular}

$\mathrm{nl}$... new line, ${ }^{a}$ previously unknown energy level 
Table 1 - Continued

\begin{tabular}{|c|c|c|c|c|c|}
\hline \multirow[b]{2}{*}{$\lambda / \AA$} & \multirow[b]{2}{*}{ SNR } & \multicolumn{2}{|r|}{ Even } & \multicolumn{2}{|r|}{ Odd } \\
\hline & & J-value & Level Energy $/ \mathrm{cm}^{-1}$ & J-value & Level Energy $/ \mathrm{cm}^{-1}$ \\
\hline 4546.682 & nl 12 & $11 / 2$ & 4866.530 & $13 / 2$ & 26854.427 \\
\hline 4546.713 & nl 11 & $5 / 2$ & 6451.823 & $3 / 2$ & 28439.569 \\
\hline 4548.226 & nl 4 & $9 / 2$ & 5822.905 & $7 / 2$ & 27803.401 \\
\hline 4551.246 & nl 9 & $9 / 2$ & 4432.240 & $11 / 2$ & 26398.087 \\
\hline 4552.857 & nl 21 & $9 / 2$ & 5822.905 & $11 / 2$ & 27780.979 \\
\hline 4553.500 & nl 14 & $11 / 2$ & 6714.199 & $11 / 2$ & 28669.174 \\
\hline 4554.101 & nl 8 & $15 / 2$ & 26333.170 & $15 / 2$ & 4381.121 \\
\hline 4556.661 & nl 6 & $9 / 2$ & 4432.240 & $7 / 2$ & 26371.983 \\
\hline 4557.606 & $\mathrm{nl} 4$ & $11 / 2$ & 10904.070 & $13 / 2$ & 32839.463 \\
\hline 4560.844 & nl 5 & $9 / 2$ & 5822.910 & $11 / 2$ & 27742.524 \\
\hline 4560.958 & nl 11 & $9 / 2$ & 4432.240 & $9 / 2$ & 26351.314 \\
\hline 4565.063 & nl 11 & $7 / 2$ & 9704.750 & $7 / 2$ & 31604.099 \\
\hline 4567.454 & nl 4 & $5 / 2$ & 6451.823 & $7 / 2$ & 28339.724 \\
\hline 4571.334 & $\mathrm{nl} 15$ & $13 / 2$ & 7951.338 & $15 / 2$ & 29820.680 \\
\hline 4578.717 & nl 4 & $9 / 2$ & 8029.290 & $9 / 2$ & 29863.351 \\
\hline 4584.435 & 9 & $7 / 2$ & 7617.455 & $9 / 2$ & 29424.282 \\
\hline 4586.971 & nl 5 & $13 / 2$ & 7951.338 & $13 / 2$ & 29746.108 \\
\hline 4587.194 & nl 6 & $9 / 2$ & 4432.240 & $9 / 2$ & 26225.952 \\
\hline 4590.878 & nl 18 & $11 / 2$ & 6892.949 & $11 / 2$ & 28669.174 \\
\hline 4598.951 & nl 178 & $11 / 2$ & 24584.745 & $13 / 2$ & 2846.744 \\
\hline 4601.217 & nl 4 & $9 / 2$ & 4432.240 & $11 / 2$ & 26159.548 \\
\hline 4605.314 & $\mathrm{nl} 15$ & $7 / 2$ & 9704.750 & $5 / 2$ & 31412.750 \\
\hline 4614.926 & nl 6 & $13 / 2$ & 7951.338 & $11 / 2$ & 29614.101 \\
\hline 4615.381 & nl 12 & $11 / 2$ & 6714.199 & $13 / 2$ & 28374.815 \\
\hline 4616.371 & nl 7 & $7 / 2$ & 6535.587 & $7 / 2$ & 28191.557 \\
\hline 4617.572 & nl 7 & $13 / 2$ & 11462.858 & $15 / 2$ & 33113.358 \\
\hline 4625.019 & $\mathrm{nl} 4$ & $11 / 2$ & 4866.530 & $9 / 2$ & 26482.007 \\
\hline 4628.172 & nl 9 & $11 / 2$ & 6313.239 & $9 / 2$ & 27913.990 \\
\hline 4628.809 & nl 14 & $13 / 2$ & 7951.338 & $15 / 2$ & 29549.117 \\
\hline 4631.028 & nl 5 & $13 / 2$ & 14328.241 & $11 / 2$ & 35915.701 \\
\hline 4631.674 & nl 14 & $17 / 2$ & 10532.001 & $15 / 2$ & 32116.407 \\
\hline 4632.908 & nl 14 & $15 / 2$ & 9745.420 & $15 / 2$ & 31324.084 \\
\hline 4634.700 & 9 & $7 / 2$ & 7617.455 & $7 / 2$ & 29187.782 \\
\hline 4636.278 & 10 & $11 / 2$ & 6714.199 & $9 / 2$ & 28277.184 \\
\hline 4639.887 & 15 & $11 / 2$ & 6714.199 & $9 / 2$ & 28260.413 \\
\hline 4640.071 & nl 37 & $9 / 2$ & 5822.905 & $9 / 2$ & 27368.267 \\
\hline 4641.986 & 8 & $11 / 2$ & 6714.199 & $13 / 2$ & 28250.672 \\
\hline 4642.487 & nl 7 & $9 / 2$ & 5822.905 & $7 / 2$ & 27357.054 \\
\hline 4644.745 & nl 8 & $11 / 2$ & 4866.530 & $13 / 2$ & 26390.208 \\
\hline 4651.668 & 20 & $7 / 2$ & 6535.587 & $7 / 2$ & 28027.233 \\
\hline 4653.786 & 8 & $11 / 2$ & 6892.949 & $13 / 2$ & 28374.815 \\
\hline 4656.848 & 11 & $11 / 2$ & 6313.239 & $11 / 2$ & 27780.979 \\
\hline 4660.254 & 8 & $17 / 2$ & 10532.001 & $17 / 2$ & 31984.040 \\
\hline 4662.205 & 15 & $9 / 2$ & 4432.240 & $9 / 2$ & 25875.314 \\
\hline 4664.728 & 40 & $11 / 2$ & 6714.199 & $9 / 2$ & 28145.675 \\
\hline 4665.961 & nl 17 & $13 / 2$ & 7630.170 & $13 / 2$ & 29055.975 \\
\hline 4670.172 & nl 3 & $13 / 2$ & 7951.350 & $13 / 2$ & 29357.903 \\
\hline
\end{tabular}

$\mathrm{nl}$... new line, ${ }^{a}$ previously unknown energy level 
Table 1 - Continued

\begin{tabular}{|c|c|c|c|c|c|}
\hline \multirow[b]{2}{*}{$\lambda / \AA$} & \multirow[b]{2}{*}{ SNR } & \multicolumn{2}{|r|}{ Even } & \multicolumn{2}{|r|}{ Odd } \\
\hline & & J-value & Level Energy $/ \mathrm{cm}^{-1}$ & $\mathrm{~J}$-value & Level Energy $/ \mathrm{cm}^{-1}$ \\
\hline 4671.245 & 15 & $9 / 2$ & 5822.905 & $7 / 2$ & 27224.482 \\
\hline 4674.505 & 15 & $11 / 2$ & 6313.239 & $11 / 2$ & 27699.892 \\
\hline 4675.033 & 40 & $11 / 2$ & 6892.949 & $9 / 2$ & 28277.184 \\
\hline 4675.100 & 9 & $11 / 2$ & 6714.220 & $9 / 2$ & 28098.250 \\
\hline 4675.480 & 10 & $15 / 2$ & 8363.916 & $13 / 2$ & 29746.108 \\
\hline 4677.769 & nl 16 & $7 / 2$ & 7617.455 & $7 / 2$ & 28989.185 \\
\hline 4680.464 & 25 & $11 / 2$ & 4866.530 & $9 / 2$ & 26225.952 \\
\hline 4682.023 & 20 & $9 / 2$ & 4432.240 & $11 / 2$ & $25789 / 252$ \\
\hline 4682.197 & 22 & $5 / 2$ & 6451.823 & $7 / 2$ & 27803.341 \\
\hline 4683.201 & $\mathrm{nl} 4$ & $13 / 2$ & 6603.606 & $15 / 2$ & 27950.545 \\
\hline 4686.980 & $\mathrm{nl} 6$ & $11 / 2$ & 4866.530 & $13 / 2$ & 26196.260 \\
\hline 4687.540 & 10 & $13 / 2$ & 7951.338 & $15 / 2$ & 29278.510 \\
\hline 4688.519 & 10 & $11 / 2$ & 6714.199 & $13 / 2$ & 28036.928 \\
\hline 4688.562 & 25 & $13 / 2$ & 7951.338 & $11 / 2$ & 29273.796 \\
\hline 4689.722 & nl 5 & $15 / 2$ & 9745.420 & $15 / 2$ & 31062.660 \\
\hline 4689.831 & 8 & $13 / 2$ & 10470.300 & $11 / 2$ & 31787.102 \\
\hline 4690.018 & 10 & $11 / 2$ & 6313.239 & $13 / 2$ & 27629.152 \\
\hline 4690.068 & 12 & $9 / 2$ & 8029.290 & $7 / 2$ & 29344.977 \\
\hline 4691.779 & 25 & $13 / 2$ & 7630.147 & $11 / 2$ & 28938.062 \\
\hline 4691.952 & nl 9 & $5 / 2$ & 6451.823 & $5 / 2$ & 27758.950 \\
\hline 4692.652 & 55 & $11 / 2$ & 4866.530 & $9 / 2$ & 26170.480 \\
\hline 4693.661 & nl 9 & $9 / 2$ & 5822.890 & $9 / 2$ & 27122.250 \\
\hline 4695.201 & nl 6 & $17 / 2$ & 9770.330 & $15 / 2$ & 31062.660 \\
\hline 4697.998 & 45 & $11 / 2$ & 6313.239 & $9 / 2$ & 27592.946 \\
\hline 4705.959 & 15 & $13 / 2$ & 7951.338 & $11 / 2$ & 29195.047 \\
\hline 4708.704 & nl 8 & $13 / 2$ & 7951.338 & $15 / 2$ & 29182.662 \\
\hline 4711.327 & 8 & $9 / 2$ & 8643.839 & $9 / 2$ & 29863.351 \\
\hline 4714.477 & $\mathrm{nl} 6$ & $11 / 2$ & 6892.950 & $11 / 2$ & 28098.267 \\
\hline 4714.548 & nl 8 & $11 / 2$ & 6714.199 & $11 / 2$ & 27919.207 \\
\hline 4715.227 & 30 & $9 / 2$ & 4432.240 & $9 / 2$ & 25634.194 \\
\hline 4715.421 & 15 & $9 / 2$ & 5822.905 & $7 / 2$ & 27023.987 \\
\hline 4717.314 & nl 3 & $5 / 2$ & 11107.690 & $5 / 2$ & 32300.347 \\
\hline 4718.618 & nl 20 & $11 / 2$ & 4866.530 & $11 / 2$ & 26053.248 \\
\hline 4720.774 & 10 & $19 / 2$ & 11151.490 & $17 / 2$ & 32328.452 \\
\hline 4725.877 & $\mathrm{nl} 8$ & $9 / 2$ & 9105.035 & $9 / 2$ & 30259.211 \\
\hline 4726.144 & 12 & $13 / 2$ & 9464.460 & $11 / 2$ & 30617.463 \\
\hline 4726.244 & 10 & $11 / 2$ & 22529.130 & $11 / 2$ & 1376.605 \\
\hline 4730.222 & 10 & $11 / 2$ & 8829.078 & $9 / 2$ & 29963.821 \\
\hline 4738.670 & 15 & $11 / 2$ & 6892.949 & $11 / 2$ & 27990.015 \\
\hline 4742.700 & 6 & $11 / 2$ & 9483.540 & $13 / 2$ & 30562.676 \\
\hline 4743.748 & 20 & $5 / 2$ & 6451.830 & $7 / 2$ & 27526.308 \\
\hline 4745.069 & 6 & $7 / 2$ & 6535.587 & $9 / 2$ & 27604.203 \\
\hline 4748.794 & 7 & $11 / 2$ & 6892.950 & $9 / 2$ & 27945.039 \\
\hline 4752.817 & 15 & $11 / 2$ & 8829.078 & $9 / 2$ & 29863.351 \\
\hline 4753.837 & 8 & $11 / 2$ & 11944.223 & $13 / 2$ & 32974.060 \\
\hline 4755.808 & 40 & $11 / 2$ & 6892.949 & $9 / 2$ & 27913.990 \\
\hline 4757.009 & 15 & $5 / 2$ & 6451.823 & $5 / 2$ & 27467.559 \\
\hline
\end{tabular}

$\mathrm{nl}$... new line, ${ }^{a}$ previously unknown energy level 
Table 1 - Continued

\begin{tabular}{|c|c|c|c|c|c|}
\hline \multirow[b]{2}{*}{$\lambda / \AA$} & \multirow[b]{2}{*}{ SNR } & \multicolumn{2}{|r|}{ Even } & \multicolumn{2}{|r|}{ Odd } \\
\hline & & J-value & Level Energy $/ \mathrm{cm}^{-1}$ & J-value & Level Energy $/ \mathrm{cm}^{-1}$ \\
\hline 4758.583 & 35 & $11 / 2$ & 4866.530 & $9 / 2$ & 25875.314 \\
\hline 4766.966 & nl 20 & $11 / 2$ & 9483.540 & $13 / 2$ & 30455.358 \\
\hline 4767.566 & 11 & $11 / 2$ & 10841.482 & $11 / 2$ & 31810.670 \\
\hline 4768.116 & 45 & $9 / 2$ & 4432.240 & $7 / 2$ & 25399.020 \\
\hline 4782.459 & 7 & $15 / 2$ & 8765.556 & $15 / 2$ & 29669.456 \\
\hline 4783.424 & 18 & $13 / 2$ & 7951.338 & $13 / 2$ & 28851.022 \\
\hline 4786.181 & 20 & $9 / 2$ & 5822.905 & $11 / 2$ & 26710.548 \\
\hline 4792.795 & 10 & $13 / 2$ & 7951.338 & $13 / 2$ & 28810.160 \\
\hline 4792.854 & 20 & $7 / 2$ & 6535.587 & $9 / 2$ & 27394.149 \\
\hline 4792.909 & 25 & $13 / 2$ & 7951.338 & $11 / 2$ & 28809.661 \\
\hline 4795.241 & 50 & $7 / 2$ & 30427.864 & $9 / 2$ & 9579.832 \\
\hline 4795.426 & 15 & $9 / 2$ & 12068.109 & $9 / 2$ & 32915.480 \\
\hline 4796.845 & 20 & $11 / 2$ & 6313.239 & $13 / 2$ & 27154.450 \\
\hline 4797.956 & 20 & $7 / 2$ & 32165.955 & $9 / 2$ & 11329.710 \\
\hline 4798.394 & 6 & $17 / 2$ & 9770.330 & $17 / 2$ & 30604.830 \\
\hline 4798.809 & 30 & $7 / 2$ & 6535.587 & $9 / 2$ & 27368.267 \\
\hline 4800.796 & 7 & $11 / 2$ & 9675.040 & $11 / 2$ & 30498.989 \\
\hline 4801.742 & 20 & $5 / 2$ & 29806.252 & $3 / 2$ & 8986.444 \\
\hline 4802.040 & 20 & $13 / 2$ & 7630.147 & $13 / 2$ & 28448.810 \\
\hline 4802.883 & 55 & $11 / 2$ & 6313.239 & $11 / 2$ & 27128.251 \\
\hline 4810.925 & 15 & $5 / 2$ & 6451.823 & $5 / 2$ & 27232.039 \\
\hline 4811.731 & 8 & $5 / 2$ & 34013.314 & $7 / 2$ & 13236.599 \\
\hline 4813.538 & 12 & $9 / 2$ & 5822.905 & $7 / 2$ & 26591.841 \\
\hline 4813.833 & 8 & $11 / 2$ & 4866.530 & $9 / 2$ & 25634.194 \\
\hline 4814.255 & 12 & $11 / 2$ & 4866.530 & $11 / 2$ & 25632.372 \\
\hline 4815.667 & 35 & $7 / 2$ & 7617.455 & $5 / 2$ & 28377.208 \\
\hline 4819.510 & 6 & $17 / 2$ & 10532.001 & $15 / 2$ & 31275.181 \\
\hline 4820.562 & 22 & $5 / 2$ & 6451.823 & $7 / 2$ & 27190.496 \\
\hline 4823.343 & 6 & $7 / 2$ & 9704.750 & $7 / 2$ & 30431.510 \\
\hline 4823.790 & 12 & $11 / 2$ & 6714.199 & $9 / 2$ & 27438.992 \\
\hline 4824.328 & 6 & $11 / 2$ & 6714.199 & $11 / 2$ & 27436.682 \\
\hline 4825.813 & 70 & $9 / 2$ & 4432.240 & $7 / 2$ & 25148.347 \\
\hline 4826.325 & 20 & $9 / 2$ & 5822.905 & $11 / 2$ & 26536.814 \\
\hline 4826.944 & 20 & $11 / 2$ & 6892.949 & $9 / 2$ & 27604.203 \\
\hline 4828.477 & 12 & $9 / 2$ & 10920.380 & $11 / 2$ & 31625.080 \\
\hline 4831.422 & 35 & $15 / 2$ & 8363.916 & $13 / 2$ & 29055.974 \\
\hline 4831.495 & 25 & $9 / 2$ & 4432.240 & $7 / 2$ & 25123.984 \\
\hline 4832.069 & nl 6 & $5 / 2$ & 6451.720 & $5 / 2$ & 27141.016 \\
\hline 4833.266 & nl 11 & $11 / 2$ & 4866.530 & $9 / 2$ & 25550.693 \\
\hline 4833.361 & nl 3 & $13 / 2$ & 10266.510 & $11 / 2$ & 30950.266 \\
\hline 4834.709 & 20 & $17 / 2$ & 25059.089 & $15 / 2$ & 4381.121 \\
\hline 4842.913 & 8 & $7 / 2$ & 7617.455 & $9 / 2$ & 28260.413 \\
\hline 4843.129 & 40 & $7 / 2$ & 6535.587 & $9 / 2$ & 27177.628 \\
\hline 4849.232 & 10 & $9 / 2$ & 8320.255 & $7 / 2$ & 28936.317 \\
\hline 4852.822 & 5 & $7 / 2$ & 6535.587 & $7 / 2$ & 27136.408 \\
\hline 4853.899 & 29 & $15 / 2$ & 8363.916 & $13 / 2$ & 28960.156 \\
\hline 4855.627 & 6 & $13 / 2$ & 9464.460 & $11 / 2$ & 30053.346 \\
\hline
\end{tabular}

$\mathrm{nl}$... new line, ${ }^{a}$ previously unknown energy level 
Table 1 - Continued

\begin{tabular}{|c|c|c|c|c|c|}
\hline \multirow[b]{2}{*}{$\lambda / \AA$} & \multirow[b]{2}{*}{ SNR } & \multicolumn{2}{|r|}{ Even } & \multicolumn{2}{|r|}{ Odd } \\
\hline & & J-value & Level Energy $/ \mathrm{cm}^{-1}$ & J-value & Level Energy $/ \mathrm{cm}^{-1}$ \\
\hline 4856.907 & nl 15 & $11 / 2$ & 6714.199 & $13 / 2$ & 27297.680 \\
\hline 4857.073 & 6 & $11 / 2$ & 8829.078 & $9 / 2$ & 29411.858 \\
\hline 4857.522 & 8 & $13 / 2$ & 7630.147 & $13 / 2$ & 28211.024 \\
\hline 4857.826 & 8 & $9 / 2$ & 31029.588 & $7 / 2$ & 10449.941 \\
\hline 4858.262 & 14 & $13 / 2$ & 6603.606 & $11 / 2$ & 27181.349 \\
\hline 4861.964 & 105 & $11 / 2$ & 4866.530 & $11 / 2$ & 25428.607 \\
\hline 4862.294 & 12 & $13 / 2$ & 7630.170 & $11 / 2$ & 28190.866 \\
\hline 4864.622 & nl 11 & $13 / 2$ & 6603.606 & $13 / 2$ & 27154.447 \\
\hline 4865.990 & 20 & $13 / 2$ & 7630.147 & $13 / 2$ & 28175.209 \\
\hline 4867.384 & 12 & $15 / 2$ & 9745.420 & $13 / 2$ & $30289 / 295$ \\
\hline 4869.332 & 70 & $11 / 2$ & 6892.949 & $13 / 2$ & 27423.911 \\
\hline 4871.188 & 10 & $5 / 2$ & 6451.720 & $5 / 2$ & 26974.973 \\
\hline 4872.929 & 20 & $11 / 2$ & 9483.540 & $13 / 2$ & 29999.350 \\
\hline 4873.218 & 20 & $9 / 2$ & 5822.905 & $9 / 2$ & 26337.496 \\
\hline 4877.116 & nl 5 & $9 / 2$ & 5822.905 & $7 / 2$ & 26321.099 \\
\hline 4878.194 & nl 97 & $13 / 2$ & 6603.606 & $11 / 2$ & 27097.270 \\
\hline 4879.251 & 35 & $9 / 2$ & 4432.240 & $11 / 2$ & 24921.466 \\
\hline 4882.565 & nl 4 & $11 / 2$ & 6892.949 & $9 / 2$ & 27368.267 \\
\hline 4883.659 & nl 7 & $7 / 2$ & 7617.455 & $9 / 2$ & 28088.187 \\
\hline 4890.834 & 4 & $9 / 2$ & 11184.410 & $11 / 2$ & 31625.080 \\
\hline 4890.942 & 16 & $11 / 2$ & 6714.199 & $13 / 2$ & 27154.450 \\
\hline 4891.324 & nl 6 & $7 / 2$ & 6535.530 & $9 / 2$ & 26974.246 \\
\hline 4892.954 & 20 & $13 / 2$ & 6603.606 & $15 / 2$ & 27035.451 \\
\hline 4897.867 & 20 & $11 / 2$ & 9483.540 & $13 / 2$ & 29894.870 \\
\hline 4899.456 & 25 & $11 / 2$ & 6892.949 & $13 / 2$ & 27297.680 \\
\hline 4899.849 & nl 10 & $5 / 2$ & 29389.577 & $3 / 2$ & 8986.444 \\
\hline 4903.836 & 6 & $7 / 2$ & 6535.587 & $5 / 2$ & 26922.093 \\
\hline 4904.463 & nl 5 & $13 / 2$ & 9464.460 & $11 / 2$ & 29848.320 \\
\hline 4904.704 & 10 & $9 / 2$ & 8643.839 & $7 / 2$ & 29026.738 \\
\hline 4911.460 & 50 & $13 / 2$ & 6603.606 & $11 / 2$ & 26958.467 \\
\hline 4911.998 & 25 & $13 / 2$ & 7951.338 & $13 / 2$ & 28303.969 \\
\hline 4913.153 & 25 & $11 / 2$ & 29927.716 & $9 / 2$ & 9579.832 \\
\hline 4913.442 & 5 & $13 / 2$ & 6603.606 & $15 / 2$ & 26950.256 \\
\hline 4915.407 & 230 & $11 / 2$ & 23185.260 & $13 / 2$ & 2846.744 \\
\hline 4915.934 & 18 & $13 / 2$ & 7951.338 & $15 / 2$ & 28287.674 \\
\hline 4916.365 & 12 & $11 / 2$ & 31664.301 & $9 / 2$ & 11329.710 \\
\hline 4916.999 & 90 & $11 / 2$ & 6313.239 & $13 / 2$ & 26645.170 \\
\hline 4918.367 & 12 & $7 / 2$ & 7617.455 & $7 / 2$ & 27943.748 \\
\hline 4921.651 & nl 5 & $17 / 2$ & 9770.330 & $17 / 2$ & 30083.020 \\
\hline 4924.895 & nl 11 & $13 / 2$ & 7951.338 & $13 / 2$ & 28250.672 \\
\hline 4925.137 & 10 & $7 / 2$ & 10194.740 & $9 / 2$ & 30493.058 \\
\hline 4925.315 & 450 & $15 / 2$ & 23144.347 & $13 / 2$ & 2846.744 \\
\hline 4925.565 & 8 & $7 / 2$ & 7617.455 & $9 / 2$ & 27913.992 \\
\hline 4926.374 & 6 & $7 / 2$ & 6535.587 & $9 / 2$ & 26828.828 \\
\hline 4926.559 & 8 & $9 / 2$ & 8643.839 & $7 / 2$ & 28936.317 \\
\hline 4927.059 & 55 & $15 / 2$ & 8765.556 & $13 / 2$ & 29055.974 \\
\hline 4927.389 & 5 & $13 / 2$ & 7630.147 & $11 / 2$ & 27919.207 \\
\hline
\end{tabular}

$\mathrm{nl}$... new line, ${ }^{a}$ previously unknown energy level 
Table 1 - Continued

\begin{tabular}{|c|c|c|c|c|c|}
\hline \multirow[b]{2}{*}{$\lambda / \AA$} & \multirow[b]{2}{*}{ SNR } & \multicolumn{2}{|r|}{ Even } & \multicolumn{2}{|r|}{ Odd } \\
\hline & & J-value & Level Energy $/ \mathrm{cm}^{-1}$ & J-value & Level Energy $/ \mathrm{cm}^{-1}$ \\
\hline 4928.454 & 12 & $11 / 2$ & 6892.948 & $9 / 2$ & 27177.628 \\
\hline 4930.775 & 15 & $11 / 2$ & 6313.239 & $11 / 2$ & 26588.368 \\
\hline 4931.669 & nl 3 & $17 / 2$ & 12736.636 & $15 / 2$ & 33008.091 \\
\hline 4934.091 & 25 & $11 / 2$ & 6892.949 & $13 / 2$ & 27154.450 \\
\hline 4934.209 & nl 25 & $9 / 2$ & 5822.905 & $7 / 2$ & 26083.919 \\
\hline 4935.747 & nl 6 & $9 / 2$ & 5822.905 & $7 / 2$ & 26077.608 \\
\hline 4936.822 & nl 6 & $11 / 2$ & 6714.199 & $11 / 2$ & 26964.492 \\
\hline 4940.021 & 20 & $9 / 2$ & 4432.240 & $7 / 2$ & 24669.420 \\
\hline 4941.500 & 10 & $9 / 2$ & 8029.290 & $9 / 2$ & 28260.413 \\
\hline 4943.344 & 10 & $11 / 2$ & 6313.239 & $11 / 2$ & 26536.814 \\
\hline 4946.319 & 5 & $17 / 2$ & 10532.001 & $17 / 2$ & 30743.399 \\
\hline 4948.508 & 25 & $9 / 2$ & 8029.290 & $11 / 2$ & 28231.857 \\
\hline 4951.158 & nl 6 & $7 / 2$ & 6535.587 & $7 / 2$ & 26727.245 \\
\hline 4955.233 & 12 & $9 / 2$ & 8029.290 & $7 / 2$ & 28204.346 \\
\hline 4958.234 & nl 6 & $7 / 2$ & 28412.960 & $9 / 2$ & 8250.170 \\
\hline 4958.376 & 12 & $9 / 2$ & 8029.290 & $7 / 2$ & 28191.557 \\
\hline 4960.479 & $\mathrm{nl} 4$ & $13 / 2$ & 6603.606 & $11 / 2$ & 26757.324 \\
\hline 4960.898 & 20 & $9 / 2$ & 8643.839 & $9 / 2$ & 28795.855 \\
\hline 4961.027 & 290 & $11 / 2$ & 22998.237 & $13 / 2$ & 2846.744 \\
\hline 4962.213 & 23 & $5 / 2$ & 29133.138 & $3 / 2$ & 8986.444 \\
\hline 4962.440 & nl 3 & $15 / 2$ & 35493.283 & $13 / 2$ & 15347.433 \\
\hline 4962.959 & nl 5 & $17 / 2$ & 12736.670 & $15 / 2$ & 32880.323 \\
\hline 4963.853 & 25 & $5 / 2$ & 6451.823 & $7 / 2$ & 26591.841 \\
\hline 4964.105 & 6 & $13 / 2$ & 10423.680 & $13 / 2$ & 30562.676 \\
\hline 4964.181 & 55 & $11 / 2$ & 29718.524 & $9 / 2$ & 9579.832 \\
\hline 4968.359 & 30 & $7 / 2$ & 6535.530 & $5 / 2$ & 26657.370 \\
\hline 4971.514 & 12 & $11 / 2$ & 8829.078 & $11 / 2$ & 28938.062 \\
\hline 4974.297 & 12 & $13 / 2$ & 6603.606 & $15 / 2$ & 26701.341 \\
\hline 4977.863 & nl 7 & $13 / 2$ & 6603.606 & $11 / 2$ & 26686.944 \\
\hline 4980.473 & 28 & $17 / 2$ & 10532.001 & $17 / 2$ & 30604.830 \\
\hline 4981.232 & 6 & $13 / 2$ & 7630.147 & $11 / 2$ & 27699.892 \\
\hline 4983.128 & 8 & $13 / 2$ & 9464.450 & $11 / 2$ & 29526.570 \\
\hline 4983.605 & 25 & $13 / 2$ & 10423.680 & $15 / 2$ & 30483.514 \\
\hline 4983.729 & 5 & $11 / 2$ & 9483.540 & $13 / 2$ & 29543.242 \\
\hline 4983.929 & nl 6 & $9 / 2$ & 8029.280 & $9 / 2$ & 28088.171 \\
\hline 4984.912 & 13 & $11 / 2$ & 4866.530 & $11 / 2$ & 24921.466 \\
\hline 4990.882 & 14 & $11 / 2$ & 9268.750 & $15 / 2$ & 29299.668 \\
\hline 4991.069 & 6 & $17 / 2$ & 9770.330 & $13 / 2$ & 29800.480 \\
\hline 4992.319 & 45 & $9 / 2$ & 5822.905 & $7 / 2$ & 25848.090 \\
\hline 4992.551 & 8 & $11 / 2$ & 6313.239 & $9 / 2$ & 26337.496 \\
\hline 4995.013 & 8 & $9 / 2$ & 8029.290 & $9 / 2$ & 28043.749 \\
\hline 4995.727 & 25 & $7 / 2$ & 9918.170 & $9 / 2$ & 29929.693 \\
\hline 4998.646 & 5 & $11 / 2$ & 9483.533 & $13 / 2$ & 29483.354 \\
\hline 5002.416 & nl 6 & $13 / 2$ & 6603.606 & $11 / 2$ & 26588.368 \\
\hline 5005.950 & 8 & $7 / 2$ & 9704.750 & $7 / 2$ & 29675.409 \\
\hline 5006.330 & 6 & $21 / 2$ & 12665.074 & $19 / 2$ & 32634.220 \\
\hline 5007.259 & 10 & $13 / 2$ & 9464.460 & $11 / 2$ & 29429.898 \\
\hline
\end{tabular}

$\mathrm{nl}$... new line, ${ }^{a}$ previously unknown energy level 
Table 1 - Continued

\begin{tabular}{|c|c|c|c|c|c|}
\hline \multirow[b]{2}{*}{$\lambda / \AA$} & \multirow[b]{2}{*}{ SNR } & \multicolumn{2}{|r|}{ Even } & \multicolumn{2}{|r|}{ Odd } \\
\hline & & J-value & Level Energy $/ \mathrm{cm}^{-1}$ & J-value & Level Energy $/ \mathrm{cm}^{-1}$ \\
\hline 5009.393 & nl 15 & $9 / 2$ & 8320.255 & $9 / 2$ & 28277.184 \\
\hline 5009.867 & 6 & $9 / 2$ & 11713.249 & $7 / 2$ & 31668.036 \\
\hline 5011.381 & 25 & $7 / 2$ & 8013.104 & $5 / 2$ & 27962.119 \\
\hline 5015.918 & $\mathrm{nl} 6$ & $11 / 2$ & 6714.199 & $13 / 2$ & 26645.170 \\
\hline 5016.563 & nl 9 & $11 / 2$ & 8829.078 & $9 / 2$ & 28757.493 \\
\hline 5017.880 & 65 & $13 / 2$ & 7951.338 & $15 / 2$ & $27879 / 216$ \\
\hline 5018.268 & nl 116 & $13 / 2$ & 31918.637 & $11 / 2$ & 11997.050 \\
\hline 5023.471 & $\mathrm{nl} 5$ & $13 / 2$ & 7630.147 & $15 / 2$ & 27531.150 \\
\hline 5023.935 & 40 & $17 / 2$ & 9770.330 & $15 / 2$ & 29669.474 \\
\hline 5028.016 & 35 & $11 / 2$ & 6313.239 & $13 / 2$ & 26196.253 \\
\hline 5028.391 & nl 10 & $9 / 2$ & 8643.830 & $7 / 2$ & 28525.354 \\
\hline 5029.391 & 20 & $15 / 2$ & 9745.420 & $17 / 2$ & 29623.001 \\
\hline 5029.705 & 45 & $13 / 2$ & 6603.606 & $11 / 2$ & 26479.944 \\
\hline 5030.254 & nl 12 & $11 / 2$ & 6714.199 & $11 / 2$ & 26588.368 \\
\hline 5031.493 & nl 10 & $5 / 2$ & 6451.823 & $7 / 2$ & 26321.099 \\
\hline 5031.559 & nl 8 & $11 / 2$ & 8829.078 & $11 / 2$ & 28698.091 \\
\hline 5031.770 & 85 & $11 / 2$ & 9675.040 & $13 / 2$ & 29543.242 \\
\hline 5032.116 & 28 & $7 / 2$ & 11668.787 & $7 / 2$ & 31535.639 \\
\hline 5034.639 & 55 & $15 / 2$ & 8765.556 & $15 / 2$ & 28622.414 \\
\hline 5035.615 & 10 & $11 / 2$ & 10841.482 & $13 / 2$ & 30694.484 \\
\hline 5036.352 & 30 & $7 / 2$ & 7617.455 & $5 / 2$ & 27467.559 \\
\hline 5037.113 & 35 & $15 / 2$ & 8363.916 & $13 / 2$ & 28211.024 \\
\hline 5038.893 & 22 & $11 / 2$ & 8829.078 & $11 / 2$ & 28669.174 \\
\hline 5039.832 & 45 & $7 / 2$ & 6535.587 & $7 / 2$ & 26371.986 \\
\hline 5040.430 & nl 10 & $15 / 2$ & 8363.916 & $15 / 2$ & 28197.961 \\
\hline 5041.550 & 60 & $13 / 2$ & 7951.338 & $11 / 2$ & 27780.979 \\
\hline 5042.623 & 30 & $9 / 2$ & 8320.255 & $9 / 2$ & 28145.675 \\
\hline 5043.337 & 20 & $11 / 2$ & 6714.199 & $11 / 2$ & 26536.814 \\
\hline 5043.611 & 50 & $7 / 2$ & 7617.455 & $9 / 2$ & 27438.992 \\
\hline 5046.976 & 20 & $11 / 2$ & 9675.040 & $13 / 2$ & 29483.340 \\
\hline 5047.431 & 22 & $13 / 2$ & 7630.147 & $11 / 2$ & 27436.682 \\
\hline 5049.068 & nl 6 & $13 / 2$ & 14087.563 & $13 / 2$ & 33887.677 \\
\hline 5050.308 & nl 9 & $15 / 2$ & 11483.450 & $13 / 2$ & 31278.760 \\
\hline 5051.337 & 12 & $9 / 2$ & 11184.410 & $7 / 2$ & 30975.574 \\
\hline 5051.588 & nl 20 & $7 / 2$ & 8013.104 & $7 / 2$ & 27803.341 \\
\hline 5052.289 & nl 8 & $13 / 2$ & 16069.900 & $13 / 2$ & 35857.286 \\
\hline 5054.501 & 20 & $17 / 2$ & 9770.330 & $15 / 2$ & 29549.140 \\
\hline 5055.723 & 60 & $9 / 2$ & 8029.290 & $7 / 2$ & 27803.341 \\
\hline 5056.569 & 125 & $11 / 2$ & 4866.530 & $9 / 2$ & 24637.265 \\
\hline 5057.665 & nl 6 & $17 / 2$ & 11714.367 & $19 / 2$ & 31480.828 \\
\hline 5061.203 & 30 & $9 / 2$ & 11184.410 & $7 / 2$ & 30937.040 \\
\hline 5061.447 & nl 11 & $9 / 2$ & 8029.290 & $11 / 2$ & 27780.979 \\
\hline 5061.743 & 22 & $11 / 2$ & 8829.078 & $9 / 2$ & 28579.611 \\
\hline 5064.405 & nl 12 & $17 / 2$ & 9770.330 & $19 / 2$ & 29510.473 \\
\hline 5064.968 & 45 & $15 / 2$ & 9745.420 & $13 / 2$ & 29483.340 \\
\hline 5065.286 & 14 & $11 / 2$ & 9483.540 & $11 / 2$ & 29220.280 \\
\hline 5068.703 & nl 12 & $9 / 2$ & 8320.255 & $9 / 2$ & 28043.749 \\
\hline
\end{tabular}

$\mathrm{nl}$... new line, ${ }^{a}$ previously unknown energy level 
Table 1 - Continued

\begin{tabular}{|c|c|c|c|c|c|}
\hline \multirow[b]{2}{*}{$\lambda / \AA$} & \multirow[b]{2}{*}{ SNR } & \multicolumn{2}{|r|}{ Even } & \multicolumn{2}{|r|}{ Odd } \\
\hline & & J-value & Level Energy $/ \mathrm{cm}^{-1}$ & J-value & Level Energy $/ \mathrm{cm}^{-1}$ \\
\hline 5074.000 & 170 & $11 / 2$ & 4866.530 & $9 / 2$ & 24569.356 \\
\hline 5075.068 & nl 5 & $15 / 2$ & 9745.420 & $13 / 2$ & 29444.080 \\
\hline 5075.586 & 135 & $11 / 2$ & 8829.090 & $13 / 2$ & 28525.569 \\
\hline 5075.865 & 270 & $9 / 2$ & 5822.905 & $9 / 2$ & 25518.489 \\
\hline 5076.327 & 20 & $13 / 2$ & 13467.459 & $13 / 2$ & 33161.246 \\
\hline 5077.880 & 17 & $11 / 2$ & 8829.078 & $11 / 2$ & 28516.848 \\
\hline 5077.984 & nl 8 & $13 / 2$ & 10423.680 & $11 / 2$ & 30110.987 \\
\hline 5078.617 & nl 11 & $7 / 2$ & 14045.708 & $5 / 2$ & 33730.711 \\
\hline 5078.881 & 30 & $11 / 2$ & 6714.199 & $11 / 2$ & 26398.087 \\
\hline 5079.266 & 280 & $11 / 2$ & 22529.130 & $13 / 2$ & 2846.744 \\
\hline 5081.627 & 15 & $5 / 2$ & 30123.185 & $7 / 2$ & 10449.941 \\
\hline 5081.689 & nl 5 & $15 / 2$ & 8363.916 & $13 / 2$ & 28036.928 \\
\hline 5081.931 & 15 & $13 / 2$ & 10470.300 & $13 / 2$ & 30142.519 \\
\hline 5082.147 & nl 3 & $11 / 2$ & 9675.040 & $9 / 2$ & 29346.312 \\
\hline 5082.164 & 10 & $13 / 2$ & 10423.680 & $11 / 2$ & 30094.818 \\
\hline 5082.312 & 25 & $9 / 2$ & 8029.290 & $11 / 2$ & 27699.892 \\
\hline 5082.712 & 12 & $13 / 2$ & 12118.054 & $11 / 2$ & 31787.102 \\
\hline 5082.780 & 25 & $7 / 2$ & 10194.740 & $9 / 2$ & 29863.299 \\
\hline 5083.105 & 15 & $13 / 2$ & 7630.147 & $13 / 2$ & 27297.680 \\
\hline 5083.707 & nl 9 & $17 / 2$ & 12736.636 & $15 / 2$ & 32401.839 \\
\hline 5084.975 & 20 & $9 / 2$ & 12068.109 & $11 / 2$ & 31728.490 \\
\hline 5087.299 & nl 14 & $5 / 2$ & 6451.720 & $5 / 2$ & 26103.032 \\
\hline 5087.444 & nl 16 & $7 / 2$ & 9704.750 & $9 / 2$ & 29355.545 \\
\hline 5088.550 & nl 21 & $13 / 2$ & 10423.680 & $11 / 2$ & 30070.158 \\
\hline 5088.715 & 15 & $13 / 2$ & 13467.459 & $15 / 2$ & 33113.358 \\
\hline 5089.229 & 35 & $11 / 2$ & 6892.949 & $11 / 2$ & 26536.814 \\
\hline 5091.956 & 17 & $9 / 2$ & 8643.839 & $9 / 2$ & 28277.184 \\
\hline 5094.097 & 35 & $9 / 2$ & 8029.290 & $7 / 2$ & 27654.383 \\
\hline 5095.476 & 40 & $9 / 2$ & 5822.890 & $9 / 2$ & 25442.742 \\
\hline 5096.794 & 60 & $13 / 2$ & 6603.606 & $11 / 2$ & 26218.316 \\
\hline 5096.989 & 15 & $11 / 2$ & 10841.482 & $13 / 2$ & 30455.358 \\
\hline 5099.136 & 60 & $9 / 2$ & 5822.905 & $11 / 2$ & 25428.607 \\
\hline 5100.548 & nl 13 & $7 / 2$ & 8013.104 & $7 / 2$ & 27613.379 \\
\hline 5102.010 & nl 6 & $11 / 2$ & 13250.690 & $11 / 2$ & 32845.298 \\
\hline 5102.427 & nl 16 & $9 / 2$ & 9105.035 & $11 / 2$ & 28698.091 \\
\hline 5103.453 & nl 25 & $11 / 2$ & 6313.239 & $13 / 2$ & 25902.359 \\
\hline 5104.006 & 35 & $11 / 2$ & 6892.949 & $11 / 2$ & 26479.944 \\
\hline 5104.101 & 35 & $15 / 2$ & 8363.916 & $15 / 2$ & 27950.545 \\
\hline 5105.878 & nl 9 & $13 / 2$ & 7951.338 & $15 / 2$ & 27531.150 \\
\hline 5109.078 & nl 60 & $7 / 2$ & 6535.530 & $5 / 2$ & 26103.032 \\
\hline 5110.653 & nl 14 & $15 / 2$ & 11483.450 & $13 / 2$ & 31044.990 \\
\hline 5110.997 & nl 5 & $7 / 2$ & 7617.455 & $9 / 2$ & 27177.628 \\
\hline 5114.261 & 50 & $9 / 2$ & 8643.839 & $7 / 2$ & 28191.557 \\
\hline 5114.450 & 20 & $9 / 2$ & 8643.839 & $11 / 2$ & 28190.835 \\
\hline 5115.064 & 15 & $9 / 2$ & 12519.718 & $9 / 2$ & 32064.342 \\
\hline 5115.512 & 15 & $17 / 2$ & 12736.636 & $15 / 2$ & 32279.575 \\
\hline 5115.752 & nl 14 & $7 / 2$ & 6535.587 & $7 / 2$ & 26077.608 \\
\hline
\end{tabular}

$\mathrm{nl}$... new line, ${ }^{a}$ previously unknown energy level 
Table 1 - Continued

\begin{tabular}{|c|c|c|c|c|c|}
\hline \multirow[b]{2}{*}{$\lambda / \AA$} & \multirow[b]{2}{*}{ SNR } & \multicolumn{2}{|r|}{ Even } & \multicolumn{2}{|r|}{ Odd } \\
\hline & & J-value & Level Energy $/ \mathrm{cm}^{-1}$ & J-value & Level Energy $/ \mathrm{cm}^{-1}$ \\
\hline 5117.291 & 380 & $15 / 2$ & 9745.420 & $17 / 2$ & 29281.511 \\
\hline 5118.082 & 40 & $15 / 2$ & 9745.420 & $15 / 2$ & 29278.510 \\
\hline 5119.475 & nl 2 & $3 / 2$ & 9650.060 & $3 / 2$ & 29177.805 \\
\hline 5123.819 & 15 & $17 / 2$ & 9770.330 & $17 / 2$ & 29281.511 \\
\hline 5124.750 & 10 & $9 / 2$ & 5822.905 & $9 / 2$ & 25330.615 \\
\hline 5125.508 & 30 & $7 / 2$ & 7617.455 & $9 / 2$ & 27122.282 \\
\hline 5126.511 & 20 & $7 / 2$ & 29246.322 & $5 / 2$ & 9745.313 \\
\hline 5130.633 & nl 11 & $13 / 2$ & 7951.338 & $11 / 2$ & 27436.682 \\
\hline 5130.693 & nl 11 & $15 / 2$ & 8765.556 & $13 / 2$ & 28250.672 \\
\hline 5130.965 & nl 12 & $13 / 2$ & 10266.510 & $11 / 2$ & 29750.596 \\
\hline 5131.245 & nl 11 & $7 / 2$ & 9704.750 & $7 / 2$ & 29187.753 \\
\hline 5131.414 & nl 22 & $11 / 2$ & 9483.540 & $9 / 2$ & 28965.900 \\
\hline 5131.780 & nl 7 & $15 / 2$ & 8363.916 & $17 / 2$ & 27844.904 \\
\hline 5132.931 & 25 & $11 / 2$ & 9483.540 & $13 / 2$ & 28960.155 \\
\hline 5133.996 & nl 38 & $13 / 2$ & 7951.350 & $13 / 2$ & 27423.929 \\
\hline 5134.331 & nl 15 & $11 / 2$ & 6313.239 & $11 / 2$ & $25789 / 252$ \\
\hline 5136.270 & 55 & $11 / 2$ & 30793.720 & $9 / 2$ & 11329.710 \\
\hline 5136.554 & 25 & $11 / 2$ & 10841.482 & $9 / 2$ & 30304.328 \\
\hline 5137.191 & 30 & $9 / 2$ & 8643.839 & $7 / 2$ & 28104.309 \\
\hline 5137.746 & $\mathrm{nl} 4$ & $11 / 2$ & 6892.949 & $9 / 2$ & 26351.314 \\
\hline 5138.297 & 85 & $11 / 2$ & 6714.199 & $9 / 2$ & 26170.480 \\
\hline 5140.456 & 12 & $11 / 2$ & 8829.078 & $9 / 2$ & 28277.184 \\
\hline 5141.065 & 35 & $5 / 2$ & 6451.823 & $7 / 2$ & 25897.628 \\
\hline 5145.418 & 15 & $11 / 2$ & 9268.750 & $11 / 2$ & 28698.103 \\
\hline 5145.583 & 20 & $13 / 2$ & 7951.350 & $11 / 2$ & 27380.069 \\
\hline 5146.033 & 35 & $9 / 2$ & 4432.240 & $11 / 2$ & 23859.280 \\
\hline 5148.025 & 18 & $7 / 2$ & 6535.530 & $5 / 2$ & 25955.285 \\
\hline 5153.249 & 50 & $9 / 2$ & 8643.830 & $9 / 2$ & 28043.749 \\
\hline 5154.471 & 45 & $13 / 2$ & 7630.170 & $13 / 2$ & 27025.398 \\
\hline 5155.889 & nl 19 & $7 / 2$ & 8013.108 & $7 / 2$ & 27403.063 \\
\hline 5157.619 & 45 & $9 / 2$ & 8643.830 & $7 / 2$ & 28027.240 \\
\hline 5158.005 & 28 & $11 / 2$ & 8829.090 & $13 / 2$ & 28211.019 \\
\hline 5159.359 & nl 3 & $13 / 2$ & 11462.858 & $11 / 2$ & 30839.845 \\
\hline 5159.437 & nl 6 & $13 / 2$ & 6603.630 & $11 / 2$ & 25980.151 \\
\hline 5160.055 & nl 7 & $7 / 2$ & 12049.463 & $5 / 2$ & 31423.759 \\
\hline 5160.198 & nl 12 & $9 / 2$ & 8029.280 & $7 / 2$ & 27403.063 \\
\hline 5160.433 & 12 & $7 / 2$ & 10194.740 & $9 / 2$ & 29567.550 \\
\hline 5162.555 & nl 11 & $9 / 2$ & 8029.280 & $9 / 2$ & 27394.123 \\
\hline 5162.628 & nl 8 & $7 / 2$ & 12049.463 & $9 / 2$ & 31414.112 \\
\hline 5163.382 & 20 & $11 / 2$ & 8829.090 & $11 / 2$ & 28190.866 \\
\hline 5163.992 & $\mathrm{nl} 4$ & $15 / 2$ & 11483.450 & $13 / 2$ & 30842.933 \\
\hline 5164.391 & nl 8 & $11 / 2$ & 13035.713 & $11 / 2$ & 32393.690 \\
\hline 5166.709 & 20 & $17 / 2$ & 12736.636 & $19 / 2$ & 32085.996 \\
\hline 5166.976 & 25 & $17 / 2$ & 11714.367 & $15 / 2$ & 31062.650 \\
\hline 5167.167 & nl 5 & $13 / 2$ & 10266.510 & $11 / 2$ & 29614.101 \\
\hline 5167.496 & 20 & $13 / 2$ & 7951.350 & $13 / 2$ & 27297.717 \\
\hline 5167.541 & 50 & $9 / 2$ & 8643.830 & $11 / 2$ & 27990.060 \\
\hline
\end{tabular}

$\mathrm{nl}$... new line, ${ }^{a}$ previously unknown energy level 
Table 1 - Continued

\begin{tabular}{|c|c|c|c|c|c|}
\hline \multirow[b]{2}{*}{$\lambda / \AA$} & \multirow[b]{2}{*}{ SNR } & \multicolumn{2}{|r|}{ Even } & \multicolumn{2}{|r|}{ Odd } \\
\hline & & J-value & Level Energy $/ \mathrm{cm}^{-1}$ & J-value & Level Energy $/ \mathrm{cm}^{-1}$ \\
\hline 5169.444 & nl 10 & $11 / 2$ & 6714.210 & $11 / 2$ & 26053.238 \\
\hline 5171.980 & nl 17 & $5 / 2$ & 11646.260 & $7 / 2$ & 30975.574 \\
\hline 5172.688 & nl 8 & $13 / 2$ & 10423.680 & $11 / 2$ & 29750.596 \\
\hline 5173.086 & 40 & $9 / 2$ & 5822.890 & $7 / 2$ & 25148.315 \\
\hline 5173.103 & nl 47 & $11 / 2$ & 6892.950 & $11 / 2$ & 26218.327 \\
\hline 5173.528 & nl 7 & $5 / 2$ & 11107.690 & $7 / 2$ & 30431.510 \\
\hline 5174.777 & 110 & $11 / 2$ & 6313.239 & $11 / 2$ & 25632.322 \\
\hline 5177.821 & 15 & $11 / 2$ & 29718.524 & $13 / 2$ & 10410.760 \\
\hline 5178.660 & nl 8 & $7 / 2$ & 7617.440 & $5 / 2$ & 26922.023 \\
\hline 5179.969 & nl 6 & $17 / 2$ & 11714.367 & $17 / 2$ & 31014.122 \\
\hline 5180.481 & 25 & $15 / 2$ & 9745.420 & $15 / 2$ & 29043.204 \\
\hline 5183.491 & 100 & $9 / 2$ & 19286.645 & $9 / 2$ & 0 \\
\hline 5183.719 & 25 & $9 / 2$ & 9105.035 & $7 / 2$ & 28390.848 \\
\hline $5189 / 277$ & nl 19 & $13 / 2$ & 10266.510 & $15 / 2$ & 29549.140 \\
\hline 5185.335 & 20 & $11 / 2$ & 11282.870 & $13 / 2$ & 30562.700 \\
\hline 5185.439 & nl 20 & $15 / 2$ & 10466.730 & $13 / 2$ & 29746.124 \\
\hline 5186.016 & 22 & $9 / 2$ & 8643.830 & $7 / 2$ & 27921.182 \\
\hline 5186.417 & nl 6 & $13 / 2$ & 10470.300 & $13 / 2$ & 29746.124 \\
\hline 5187.176 & nl 12 & $17 / 2$ & 9770.330 & $15 / 2$ & 29043.204 \\
\hline 5187.600 & nl 11 & $15 / 2$ & 8765.570 & $13 / 2$ & 28036.928 \\
\hline 5188.367 & 20 & $17 / 2$ & 10532.001 & $15 / 2$ & 29800.480 \\
\hline 5189.033 & 20 & $11 / 2$ & 10904.070 & $11 / 2$ & 30170.090 \\
\hline 5189.850 & 30 & $11 / 2$ & 9675.040 & $11 / 2$ & 28938.062 \\
\hline 5190.494 & 120 & $13 / 2$ & 7951.350 & $15 / 2$ & 27212.011 \\
\hline 5192.500 & 45 & $5 / 2$ & 6451.823 & $7 / 2$ & 25705.204 \\
\hline 5193.356 & 47 & $7 / 2$ & 13454.250 & $9 / 2$ & 32704.290 \\
\hline 5196.752 & nl 22 & $11 / 2$ & 6313.239 & $9 / 2$ & 25550.693 \\
\hline 5202.937 & 20 & $11 / 2$ & 9483.540 & $11 / 2$ & 28698.103 \\
\hline 5208.202 & nl 10 & $9 / 2$ & 12519.718 & $7 / 2$ & 31714.917 \\
\hline 5208.940 & nl 12 & $9 / 2$ & 5822.905 & $9 / 2$ & 25015.330 \\
\hline 5210.956 & 130 & $15 / 2$ & 8765.570 & $15 / 2$ & 27950.542 \\
\hline 5212.059 & nl 5 & $13 / 2$ & 6603.606 & $11 / 2$ & $25789 / 252$ \\
\hline 5213.404 & 100 & $11 / 2$ & 9675.040 & $13 / 2$ & 28851.059 \\
\hline 5216.523 & $\mathrm{nl} 6$ & $7 / 2$ & 8013.108 & $9 / 2$ & 27177.628 \\
\hline 5219.312 & 16 & $15 / 2$ & 9745.420 & $15 / 2$ & 28899.719 \\
\hline 5220.665 & nl 14 & $11 / 2$ & 10904.070 & $11 / 2$ & 30053.361 \\
\hline 5223.241 & nl 12 & $13 / 2$ & 10423.680 & $15 / 2$ & 29563.560 \\
\hline 5223.986 & 75 & $9 / 2$ & 8643.830 & $11 / 2$ & 27780.979 \\
\hline 5225.915 & nl 13 & $9 / 2$ & 13822.500 & $7 / 2$ & 32952.378 \\
\hline 5226.088 & 34 & $11 / 2$ & 6313.239 & $9 / 2$ & 25442.695 \\
\hline 5227.178 & nl 12 & $13 / 2$ & 10423.680 & $15 / 2$ & 29549.140 \\
\hline 5227.804 & nl 33 & $7 / 2$ & 8013.140 & $7 / 2$ & 27136.408 \\
\hline 5231.691 & 115 & $15 / 2$ & 8765.570 & $15 / 2$ & 27874.491 \\
\hline 5232.600 & 22 & $15 / 2$ & 9745.420 & $13 / 2$ & 28851.059 \\
\hline 5233.350 & nl 7 & $13 / 2$ & 10423.680 & $11 / 2$ & 29526.571 \\
\hline 5233.563 & nl 8 & $9 / 2$ & 10356.710 & $11 / 2$ & 29458.910 \\
\hline 5235.476 & nl 7 & $17 / 2$ & 12736.636 & $15 / 2$ & 31831.809 \\
\hline
\end{tabular}

$\mathrm{nl}$... new line, ${ }^{a}$ previously unknown energy level 
Table 1 - Continued

\begin{tabular}{|c|c|c|c|c|c|}
\hline \multirow[b]{2}{*}{$\lambda / \AA$} & \multirow[b]{2}{*}{ SNR } & \multicolumn{2}{|r|}{ Even } & \multicolumn{2}{|r|}{ Odd } \\
\hline & & J-value & Level Energy $/ \mathrm{cm}^{-1}$ & J-value & Level Energy $/ \mathrm{cm}^{-1}$ \\
\hline 5236.068 & 25 & $9 / 2$ & 8029.280 & $9 / 2$ & 27122.282 \\
\hline 5236.853 & 25 & $11 / 2$ & 8829.090 & $11 / 2$ & 27919.207 \\
\hline 5237.650 & nl 11 & $11 / 2$ & 6892.949 & $11 / 2$ & 25980.167 \\
\hline 5240.541 & 35 & $9 / 2$ & 5822.905 & $7 / 2$ & 24899.636 \\
\hline 5244.116 & nl 9 & $9 / 2$ & 9105.017 & $11 / 2$ & 28168.719 \\
\hline 5247.222 & 10 & $13 / 2$ & 9464.450 & $11 / 2$ & 28516.842 \\
\hline 5252.448 & 90 & $5 / 2$ & 6451.823 & $7 / 2$ & 25485.269 \\
\hline 5254.881 & 12 & $15 / 2$ & 12250.534 & $15 / 2$ & 31275.165 \\
\hline 5257.536 & 135 & $13 / 2$ & 7630.147 & $13 / 2$ & 26645.170 \\
\hline 5257.855 & nl 18 & $13 / 2$ & 7951.350 & $13 / 2$ & 26965.207 \\
\hline 5259.274 & 135 & $7 / 2$ & 19008.740 & $9 / 2$ & 0 \\
\hline 5259.889 & nl 6 & $9 / 2$ & 13974.730 & $11 / 2$ & 32981.247 \\
\hline 5260.015 & nl 8 & $11 / 2$ & 11944.223 & $11 / 2$ & 30950.266 \\
\hline 5260.554 & 20 & $11 / 2$ & 9483.540 & $13 / 2$ & 28487.647 \\
\hline 5261.896 & 14 & $9 / 2$ & 9105.017 & $7 / 2$ & 28104.309 \\
\hline 5266.365 & nl 12 & $11 / 2$ & 12658.416 & $13 / 2$ & 31641.506 \\
\hline 5267.660 & 200 & $17 / 2$ & 10532.001 & $19 / 2$ & 29510.473 \\
\hline 5267.971 & nl 5 & $15 / 2$ & 10466.730 & $13 / 2$ & 29444.080 \\
\hline 5270.007 & 20 & $13 / 2$ & 10423.680 & $15 / 2$ & 29393.693 \\
\hline 5272.696 & nl 16 & $9 / 2$ & 8643.839 & $9 / 2$ & 27604.203 \\
\hline 5275.824 & 35 & $9 / 2$ & 8643.839 & $9 / 2$ & 27592.946 \\
\hline 5276.623 & 12 & $11 / 2$ & 30692.414 & $13 / 2$ & 11746.340 \\
\hline 5280.101 & 24 & $15 / 2$ & 8363.916 & $13 / 2$ & 27297.680 \\
\hline 5280.717 & nl 15 & $19 / 2$ & 11151.490 & $17 / 2$ & 30083.020 \\
\hline 5284.800 & nl 9 & $9 / 2$ & 8320.255 & $9 / 2$ & 27237.190 \\
\hline 5285.018 & nl 8 & $13 / 2$ & 10266.510 & $15 / 2$ & 29182.662 \\
\hline 5285.628 & 1650 & $13 / 2$ & 20290.580 & $11 / 2$ & 1376.605 \\
\hline 5286.635 & 30 & $13 / 2$ & 9464.460 & $13 / 2$ & 28374.815 \\
\hline 5287.547 & 85 & $7 / 2$ & 6535.587 & $9 / 2$ & 25442.695 \\
\hline 5287.725 & nl 22 & $11 / 2$ & 9268.741 & $13 / 2$ & 28175.209 \\
\hline 5289.631 & nl 13 & $11 / 2$ & 9675.040 & $13 / 2$ & 28574.696 \\
\hline 5290.536 & 120 & $11 / 2$ & 6313.239 & $11 / 2$ & 25209.670 \\
\hline 5290.946 & 52 & $13 / 2$ & 6603.606 & $15 / 2$ & 25498.559 \\
\hline 5291.885 & nl 22 & $11 / 2$ & 6892.949 & $11 / 2$ & $25789 / 252$ \\
\hline 5292.283 & nl 208 & $9 / 2$ & 18890.180 & $9 / 2$ & 0 \\
\hline 5293.670 & 17 & $5 / 2$ & 16059.899 & $7 / 2$ & 34945.103 \\
\hline 5299.787 & 100 & $7 / 2$ & 6535.587 & $7 / 2$ & 25399.020 \\
\hline 5300.035 & nl 13 & $7 / 2$ & 11274.180 & $7 / 2$ & 30136.763 \\
\hline 5303.009 & nl 10 & $3 / 2$ & 11911.437 & $5 / 2$ & 30763.338 \\
\hline 5303.421 & nl 15 & $11 / 2$ & 9675.040 & $13 / 2$ & 28525.548 \\
\hline 5306.517 & $\mathrm{nl} 10$ & $13 / 2$ & 9464.460 & $13 / 2$ & 28303.969 \\
\hline 5306.583 & 35 & $7 / 2$ & 9918.170 & $9 / 2$ & 28757.493 \\
\hline 5306.742 & 40 & $9 / 2$ & 9105.035 & $7 / 2$ & 27943.748 \\
\hline 5307.207 & 40 & $11 / 2$ & 6714.199 & $11 / 2$ & 25551.259 \\
\hline 5308.816 & 160 & $13 / 2$ & 6603.606 & $13 / 2$ & 25434.959 \\
\hline 5309.716 & nl 9 & $11 / 2$ & 11282.870 & $11 / 2$ & 30110.987 \\
\hline 5310.678 & 22 & $7 / 2$ & 8013.104 & $9 / 2$ & 26837.828 \\
\hline
\end{tabular}

$\mathrm{nl}$... new line, ${ }^{a}$ previously unknown energy level 
Table 1 - Continued

\begin{tabular}{|c|c|c|c|c|c|}
\hline \multirow[b]{2}{*}{$\lambda / \AA$} & \multirow[b]{2}{*}{ SNR } & \multicolumn{2}{|r|}{ Even } & \multicolumn{2}{|r|}{ Odd } \\
\hline & & J-value & Level Energy $/ \mathrm{cm}^{-1}$ & J-value & Level Energy $/ \mathrm{cm}^{-1}$ \\
\hline 5310.820 & nl 10 & $7 / 2$ & 11668.787 & $9 / 2$ & 30493.058 \\
\hline 5311.268 & nl 14 & $5 / 2$ & 13127.787 & $7 / 2$ & 31950.447 \\
\hline 5313.475 & nl 28 & $15 / 2$ & 10466.730 & $17 / 2$ & 29281.511 \\
\hline 5315.359 & 88 & $13 / 2$ & 10470.300 & $15 / 2$ & 29278.521 \\
\hline 5317.648 & 85 & $11 / 2$ & 8829.078 & $13 / 2$ & 27629.152 \\
\hline 5317.929 & 30 & $11 / 2$ & 27634.464 & $11 / 2$ & 8835.380 \\
\hline 5318.951 & nl 9 & $9 / 2$ & 8029.290 & $11 / 2$ & 26824.764 \\
\hline 5320.349 & 18 & $15 / 2$ & 8363.916 & $13 / 2$ & 27154.450 \\
\hline 5321.302 & nl 9 & $13 / 2$ & 13146.599 & $13 / 2$ & 31933.765 \\
\hline 5327.425 & nl 28 & $15 / 2$ & 8765.556 & $15 / 2$ & 27531.150 \\
\hline 5327.702 & 12 & $9 / 2$ & 10920.380 & $9 / 2$ & 29684.988 \\
\hline 5328.078 & nl 70 & $15 / 2$ & 23144.347 & $15 / 2$ & 4381.100 \\
\hline 5329.498 & nl 10 & $9 / 2$ & 10356.710 & $7 / 2$ & 29115.014 \\
\hline 5333.235 & nl 11 & $11 / 2$ & 4866.530 & $11 / 2$ & 23611.664 \\
\hline 5334.866 & 45 & $11 / 2$ & 6892.949 & $11 / 2$ & 25632.372 \\
\hline 5337.453 & nl 51 & $9 / 2$ & 4432.240 & $7 / 2$ & 23162.497 \\
\hline 5340.178 & nl 8 & $11 / 2$ & 6714.199 & $13 / 2$ & 25434.959 \\
\hline 5341.548 & 60 & $15 / 2$ & 10466.730 & $15 / 2$ & 29182.662 \\
\hline 5341.990 & 70 & $11 / 2$ & 6714.199 & $11 / 2$ & 25428.607 \\
\hline 5342.064 & 66 & $7 / 2$ & 8013.104 & $7 / 2$ & 26727.245 \\
\hline 5342.332 & 20 & $9 / 2$ & 8643.839 & $7 / 2$ & 27357.054 \\
\hline 5342.589 & nl 26 & $13 / 2$ & 10470.300 & $15 / 2$ & 29182.662 \\
\hline 5352.117 & nl 9 & $9 / 2$ & 8643.839 & $7 / 2$ & 27322.843 \\
\hline 5355.191 & nl 4 & $11 / 2$ & 6714.199 & $13 / 2$ & 25382.476 \\
\hline 5357.859 & nl 17 & $15 / 2$ & 11483.442 & $13 / 2$ & 30142.420 \\
\hline 5366.731 & 33 & $15 / 2$ & 10466.704 & $15 / 2$ & 29094.773 \\
\hline 5370.335 & 145 & $11 / 2$ & 6313.239 & $13 / 2$ & 24928.881 \\
\hline 5376.774 & 66 & $9 / 2$ & 8643.839 & $9 / 2$ & 27237.190 \\
\hline 5378.800 & nl 9 & $15 / 2$ & 8363.916 & $15 / 2$ & 26950.256 \\
\hline 5379.328 & nl 6 & $13 / 2$ & 10266.516 & $13 / 2$ & 28851.022 \\
\hline 5381.780 & nl 29 & $11 / 2$ & 6714.199 & $9 / 2$ & 25290.248 \\
\hline 5382.696 & nl 31 & $13 / 2$ & 10470.344 & $15 / 2$ & 29043.236 \\
\hline 5386.394 & nl 19 & $11 / 2$ & 9483.533 & $9 / 2$ & 28043.749 \\
\hline 5389.731 & 36 & $9 / 2$ & 27384.059 & $11 / 2$ & 8835.380 \\
\hline 5390.511 & 32 & $9 / 2$ & 11713.220 & $9 / 2$ & 30259.211 \\
\hline 5391.580 & 58 & $15 / 2$ & 9745.391 & $15 / 2$ & 28287.674 \\
\hline 5394.051 & 91 & $9 / 2$ & 8643.839 & $9 / 2$ & 27177.628 \\
\hline 5397.91 & 19 & $9 / 2$ & 11549.619 & $11 / 2$ & 30070.158 \\
\hline 5405.006 & 46 & $7 / 2$ & 6535.587 & $5 / 2$ & 25031.771 \\
\hline 5415.132 & 30 & $11 / 2$ & 27195.110 & $13 / 2$ & 8733.450 \\
\hline 5415.839 & nl 9 & $19 / 2$ & 13626.687 & $19 / 2$ & 32085.996 \\
\hline 5416.123 & nl 4 & $11 / 2$ & 10841.417 & $13 / 2$ & 29299.668 \\
\hline 5419.933 & 20 & $9 / 2$ & 4432.240 & $9 / 2$ & $22877.542^{a}$ \\
\hline 5423.186 & 30 & $15 / 2$ & 8765.556 & $17 / 2$ & 27199.779 \\
\hline 5423.544 & $\mathrm{nl} 10$ & $15 / 2$ & 10466.704 & $15 / 2$ & 28899.719 \\
\hline 5424.089 & 64 & $11 / 2$ & 9268.741 & $11 / 2$ & 27699.892 \\
\hline 5425.204 & nl 18 & $13 / 2$ & 10423.669 & $13 / 2$ & 28851.022 \\
\hline
\end{tabular}

$\mathrm{nl}$... new line, ${ }^{a}$ previously unknown energy level 
Table 1 - Continued

\begin{tabular}{|c|c|c|c|c|c|}
\hline \multirow[b]{2}{*}{$\lambda / \AA$} & \multirow[b]{2}{*}{ SNR } & \multicolumn{2}{|r|}{ Even } & \multicolumn{2}{|r|}{ Odd } \\
\hline & & J-value & Level Energy $/ \mathrm{cm}^{-1}$ & J-value & Level Energy $/ \mathrm{cm}^{-1}$ \\
\hline 5433.716 & nl 17 & $9 / 2$ & 8320.255 & $9 / 2$ & 26718.753 \\
\hline 5435.134 & 55 & $5 / 2$ & 9710.615 & $7 / 2$ & 28104.309 \\
\hline 5437.904 & nl 6 & $15 / 2$ & 10466.704 & $13 / 2$ & 28851.022 \\
\hline 5444.773 & nl 16 & $5 / 2$ & 10828.988 & $3 / 2$ & 29190.148 \\
\hline 5445.407 & 89 & $19 / 2$ & 11151.448 & $19 / 2$ & 29510.445 \\
\hline 5446.326 & 35 & $13 / 2$ & 10266.516 & $15 / 2$ & 28622.414 \\
\hline 5448.433 & 18 & $13 / 2$ & 28032.990 & $13 / 2$ & 9684.190 \\
\hline 5451.876 & nl 23 & $15 / 2$ & 11483.442 & $15 / 2$ & 29820.657 \\
\hline 5453.232 & 118 & $11 / 2$ & 27066.095 & $13 / 2$ & 8733.450 \\
\hline 5453.533 & 22 & $9 / 2$ & 9105.035 & $11 / 2$ & 27436.682 \\
\hline 5453.786 & 19 & $13 / 2$ & 10423.669 & $15 / 2$ & 28754.461 \\
\hline 5453.846 & 24 & $7 / 2$ & 10194.780 & $7 / 2$ & 28525.354 \\
\hline 5456.397 & 69 & $9 / 2$ & 8029.290 & $9 / 2$ & 26351.314 \\
\hline 5456.876 & 56 & $17 / 2$ & 12736.636 & $19 / 2$ & 31057.048 \\
\hline 5458.007 & 41 & $5 / 2$ & 9710.615 & $7 / 2$ & 28027.233 \\
\hline 5462.883 & 61 & $9 / 2$ & 18300.341 & $9 / 2$ & 0 \\
\hline 5467.058 & nl 7 & $11 / 2$ & 4866.530 & $9 / 2$ & 23152.820 \\
\hline 5467.974 & nl 17 & $13 / 2$ & 9464.455 & $15 / 2$ & 27747.685 \\
\hline 5469.027 & nl 5 & $15 / 2$ & 8765.556 & $15 / 2$ & 27045.255 \\
\hline 5469.506 & 189 & $13 / 2$ & 19654.728 & $11 / 2$ & 1376.605 \\
\hline 5471.965 & 79 & $15 / 2$ & 8765.556 & $15 / 2$ & 27035.451 \\
\hline 5472.499 & 22 & $9 / 2$ & 8320.255 & $11 / 2$ & 26588.368 \\
\hline 5474.995 & nl 9 & $5 / 2$ & 8737.448 & $3 / 2$ & 26997.230 \\
\hline 5498.743 & nl 10 & $9 / 2$ & 8643.839 & $11 / 2$ & 26824.764 \\
\hline 5506.379 & nl 37 & $15 / 2$ & 10466.704 & $15 / 2$ & 28622.414 \\
\hline 5513.962 & nl 13 & $7 / 2$ & 8013.104 & $7 / 2$ & 26143.830 \\
\hline 5514.111 & 75 & $9 / 2$ & 8029.290 & $11 / 2$ & 26159.548 \\
\hline 5514.574 & 55 & 4.5 & 4432.24 & 4.5 & $22560.965^{a}$ \\
\hline 5514.855 & nl 14 & $11 / 2$ & 13872.290 & $9 / 2$ & 32000.017 \\
\hline 5517.615 & nl 8 & $11 / 2$ & 10841.417 & $13 / 2$ & 28960.156 \\
\hline 5529.822 & 37 & $9 / 2$ & 30356.670 & $7 / 2$ & 12277.947 \\
\hline 5530.631 & 53 & $15 / 2$ & 8765.556 & $15 / 2$ & 26841.658 \\
\hline 5532.249 & nl 44 & $7 / 2$ & 8013.104 & $7 / 2$ & 26083.919 \\
\hline 5534.181 & 55 & $7 / 2$ & 8013.104 & $7 / 2$ & 26077.608 \\
\hline 5536.757 & nl 10 & $11 / 2$ & 10904.049 & $13 / 2$ & 28960.156 \\
\hline 5538.374 & nl 240 & $11 / 2$ & 26784.270 & $13 / 2$ & 8733.450 \\
\hline 5539.463 & 18 & $9 / 2$ & 11549.619 & $11 / 2$ & 29596.942 \\
\hline 5542.481 & nl 18 & $13 / 2$ & 10266.516 & $13 / 2$ & 28303.969 \\
\hline 5545.227 & 45 & $11 / 2$ & 6892.950 & $11 / 2$ & 24921.471 \\
\hline 5553.752 & nl 30 & $9 / 2$ & 8320.255 & $7 / 2$ & 26321.099 \\
\hline 5556.142 & $\mathrm{nl} 10$ & $7 / 2$ & 8013.104 & $9 / 2$ & 26006.208 \\
\hline 5561.672 & nl 15 & $11 / 2$ & 6714.199 & $9 / 2$ & 24689.414 \\
\hline 5568.074 & nl 8 & $11 / 2$ & 6714.199 & $13 / 2$ & 24668.738 \\
\hline 5569.608 & nl 35 & $7 / 2$ & 6535.587 & $9 / 2$ & $24485.187^{a}$ \\
\hline 5573.558 & nl 20 & $5 / 2$ & 6451.823 & $5 / 2$ & $24388.702^{a}$ \\
\hline 5573.898 & $\mathrm{nl} 35$ & $15 / 2$ & 8765.556 & $15 / 2$ & 26701.341 \\
\hline 5575.709 & nl 28 & $15 / 2$ & 9745.391 & $15 / 2$ & 27675.355 \\
\hline
\end{tabular}

$\mathrm{nl}$... new line, ${ }^{a}$ previously unknown energy level 
Table 1 - Continued

\begin{tabular}{|c|c|c|c|c|c|}
\hline \multirow[b]{2}{*}{$\lambda / \AA$} & \multirow[b]{2}{*}{ SNR } & \multicolumn{2}{|r|}{ Even } & \multicolumn{2}{|r|}{ Odd } \\
\hline & & J-value & Level Energy $/ \mathrm{cm}^{-1}$ & J-value & Level Energy $/ \mathrm{cm}^{-1}$ \\
\hline 5576.243 & nl 27 & $11 / 2$ & 8829.078 & $11 / 2$ & 26757.324 \\
\hline 5580.634 & 46 & $17 / 2$ & 14302.923 & $19 / 2$ & 32217.025 \\
\hline 5590.462 & 60 & $11 / 2$ & 4866.530 & $11 / 2$ & $22749.175^{a}$ \\
\hline 5594.760 & 82 & $9 / 2$ & 4432.240 & $7 / 2$ & $22301.160^{a}$ \\
\hline 5597.433 & nl 10 & $5 / 2$ & 9710.615 & $7 / 2$ & 27570.943 \\
\hline 5599.069 & nl 14 & $11 / 2$ & 6714.199 & $9 / 2$ & 24569.356 \\
\hline 5599.709 & 46 & $7 / 2$ & 6535.587 & $5 / 2$ & $24388.702^{a}$ \\
\hline 5608.318 & nl 14 & $7 / 2$ & 28479.761 & $7 / 2$ & 10654.110 \\
\hline 5609.809 & nl 7 & $15 / 2$ & 10466.704 & $15 / 2$ & 28287.674 \\
\hline 5610.062 & nl 38 & $11 / 2$ & 6714.199 & $11 / 2$ & 24534.366 \\
\hline 5616.325 & 27 & $15 / 2$ & 12804.483 & $17 / 2$ & 30604.778 \\
\hline 5617.018 & nl 21 & $15 / 2$ & 11483.442 & $17 / 2$ & 29281.540 \\
\hline 5617.656 & 54 & $17 / 2$ & 11714.367 & $19 / 2$ & 29510.445 \\
\hline 5619.557 & nl 16 & $7 / 2$ & 11472.423 & $9 / 2$ & 29262.534 \\
\hline 5621.481 & nl 15 & $15 / 2$ & 10466.704 & $13 / 2$ & 28250.672 \\
\hline 5624.964 & 213 & $7 / 2$ & 6535.587 & $9 / 2$ & 24308.545 \\
\hline 5627.906 & nl 9 & $9 / 2$ & 8320.255 & $7 / 2$ & 26083.919 \\
\hline 5628.833 & nl 37 & $13 / 2$ & 27444.925 & $13 / 2$ & 9684.190 \\
\hline 5629.411 & 43 & $7 / 2$ & 28412.960 & $7 / 2$ & 10654.110 \\
\hline 5630.257 & nl 18 & $13 / 2$ & 6603.606 & $11 / 2$ & 24359.861 \\
\hline 5631.009 & $\mathrm{nl} 44$ & $9 / 2$ & 5822.905 & $9 / 2$ & 23576.782 \\
\hline 5634.928 & 80 & $9 / 2$ & 10356.710 & $11 / 2$ & 28098.267 \\
\hline 5637.642 & nl 20 & $9 / 2$ & 8320.255 & $11 / 2$ & 26053.248 \\
\hline 5638.333 & nl 16 & $19 / 2$ & 28451.280 & $17 / 2$ & 10720.400 \\
\hline 5641.382 & nl 23 & $7 / 2$ & 29830.034 & $5 / 2$ & 12108.819 \\
\hline 5642.105 & nl 23 & $13 / 2$ & 7951.338 & $15 / 2$ & 25670.302 \\
\hline 5643.953 & 20 & $7 / 2$ & 7617.455 & $9 / 2$ & 25330.615 \\
\hline 5645.438 & nl 10 & $15 / 2$ & 10466.704 & $13 / 2$ & 28175.209 \\
\hline 5645.683 & 64 & $15 / 2$ & 22088.820 & $15 / 2$ & 4381.100 \\
\hline 5648.399 & nl 25 & $15 / 2$ & 11483.442 & $15 / 2$ & 29182.662 \\
\hline 5648.941 & nl 13 & $5 / 2$ & 29806.252 & $5 / 2$ & 12108.819 \\
\hline 5649.278 & 56 & $11 / 2$ & 9268.741 & $13 / 2$ & 26965.207 \\
\hline 5655.410 & nl 20 & $11 / 2$ & 11282.880 & $13 / 2$ & 28960.156 \\
\hline 5656.529 & nl 11 & $15 / 2$ & 28342.772 & $15 / 2$ & 10668.960 \\
\hline 5657.532 & 39 & $11 / 2$ & 10904.049 & $13 / 2$ & 28574.696 \\
\hline 5659.018 & 29 & $9 / 2$ & 8320.255 & $7 / 2$ & 25986.288 \\
\hline 5667.381 & nl 12 & $11 / 2$ & 9268.741 & $9 / 2$ & 26908.682 \\
\hline 5679.249 & 49 & $9 / 2$ & 8029.290 & $11 / 2$ & 25632.372 \\
\hline 5680.337 & nl 23 & $15 / 2$ & 26333.170 & $13 / 2$ & 8733.450 \\
\hline 5681.381 & nl 12 & $9 / 2$ & 10920.378 & $11 / 2$ & 28516.848 \\
\hline 5685.419 & nl 7 & $13 / 2$ & 10266.516 & $13 / 2$ & 27850.489 \\
\hline 5688.309 & nl 31 & $11 / 2$ & 6313.239 & $9 / 2$ & 23888.281 \\
\hline 5691.836 & nl 15 & $17 / 2$ & 11714.367 & $15 / 2$ & 29278.521 \\
\hline 5697.225 & nl 18 & $15 / 2$ & 14780.939 & $17 / 2$ & 32328.494 \\
\hline 5697.712 & nl 18 & $11 / 2$ & 6313.239 & $11 / 2$ & 23859.275 \\
\hline 5699.955 & 12 & $11 / 2$ & 11944.223 & $13 / 2$ & 29483.354 \\
\hline 5703.627 & 37 & $9 / 2$ & 8320.255 & $7 / 2$ & 25848.090 \\
\hline
\end{tabular}

$\mathrm{nl}$... new line, ${ }^{a}$ previously unknown energy level 
Table 1 - Continued

\begin{tabular}{|c|c|c|c|c|c|}
\hline \multirow[b]{2}{*}{$\lambda / \AA$} & \multirow[b]{2}{*}{ SNR } & \multicolumn{2}{|r|}{ Even } & \multicolumn{2}{|r|}{ Odd } \\
\hline & & J-value & Level Energy $/ \mathrm{cm}^{-1}$ & J-value & Level Energy $/ \mathrm{cm}^{-1}$ \\
\hline 5707.982 & nl 18 & $13 / 2$ & 10266.516 & $11 / 2$ & 27780.979 \\
\hline 5708.759 & 17 & $15 / 2$ & 16650.620 & $13 / 2$ & 34163.027 \\
\hline 5709.143 & nl 4 & $11 / 2$ & 27195.110 & $13 / 2$ & 9684.190 \\
\hline 5712.192 & 22 & $13 / 2$ & 12041.668 & $13 / 2$ & 29543.224 \\
\hline 5713.331 & nl 9 & $13 / 2$ & 13146.599 & $13 / 2$ & 30644.667 \\
\hline 5717.206 & nl 12 & $7 / 2$ & 11869.331 & $9 / 2$ & 29355.515 \\
\hline 5720.451 & 88 & $7 / 2$ & 28412.960 & $9 / 2$ & 10936.670 \\
\hline 5722.612 & nl 17 & $11 / 2$ & 27153.870 & $13 / 2$ & 9684.190 \\
\hline 5723.522 & nl 25 & $11 / 2$ & 6892.949 & $11 / 2$ & 24359.861 \\
\hline 5726.350 & nl 22 & $15 / 2$ & $26191.740^{a}$ & $13 / 2$ & 8733.453 \\
\hline 5726.533 & nl 11 & $7 / 2$ & 11274.244 & $5 / 2$ & 28731.850 \\
\hline 5728.378 & 70 & $17 / 2$ & 14028.722 & $19 / 2$ & 31480.828 \\
\hline 5730.189 & nl 5 & $9 / 2$ & 10356.752 & $7 / 2$ & 27803.341 \\
\hline 5731.531 & $\mathrm{nl} 5$ & $11 / 2$ & 11282.880 & $13 / 2$ & 28725.383 \\
\hline 5735.781 & 46 & $7 / 2$ & 8013.104 & $9 / 2$ & 25442.695 \\
\hline 5740.167 & nl 26 & $15 / 2$ & 11483.442 & $15 / 2$ & 28899.719 \\
\hline 5745.760 & 30 & $9 / 2$ & 8029.290 & $11 / 2$ & 25428.607 \\
\hline 5746.622 & 54 & $19 / 2$ & 29309.852 & $19 / 2$ & 11913.160 \\
\hline 5748.007 & 75 & $13 / 2$ & 6603.606 & $15 / 2$ & 23996.130 \\
\hline 5752.234 & nl 22 & $11 / 2$ & 4866.530 & $11 / 2$ & 22246.255 \\
\hline 5753.327 & 61 & $11 / 2$ & 9268.741 & $13 / 2$ & 26645.170 \\
\hline 5756.391 & nl 81 & $11 / 2$ & 8829.078 & $13 / 2$ & 26196.253 \\
\hline 5765.103 & nl 10 & $11 / 2$ & 12658.416 & $13 / 2$ & 29999.348 \\
\hline 5766.634 & 15 & $9 / 2$ & 8643.839 & $11 / 2$ & 25980.167 \\
\hline 5769.348 & nl 13 & $7 / 2$ & 28731.220 & $7 / 2$ & 11403.067 \\
\hline 5775.170 & nl 7 & $15 / 2$ & 15994.810 & $17 / 2$ & 33305.651 \\
\hline 5776.612 & 25 & $15 / 2$ & 8363.916 & $15 / 2$ & 25670.302 \\
\hline 5779.535 & nl 13 & $9 / 2$ & 10356.752 & $7 / 2$ & 27654.383 \\
\hline 5781.066 & nl 10 & $9 / 2$ & 9105.035 & $11 / 2$ & 26398.087 \\
\hline 5784.299 & 28 & $17 / 2$ & 30848.875 & $15 / 2$ & 13565.520 \\
\hline 5785.694 & 20 & $15 / 2$ & 27689.990 & $13 / 2$ & 10410.760 \\
\hline 5787.119 & nl 23 & $17 / 2$ & 9770.288 & $15 / 2$ & 27045.255 \\
\hline 5788.347 & 16 & $7 / 2$ & 28412.960 & $5 / 2$ & 11141.580 \\
\hline 5788.603 & nl 8 & $7 / 2$ & 30189.694 & $7 / 2$ & 12919.185 \\
\hline 5790.187 & 8 & $11 / 2$ & 30086.880 & $9 / 2$ & 12821.080 \\
\hline 5790.406 & nl 16 & $17 / 2$ & 9770.288 & $15 / 2$ & 27035.451 \\
\hline 5796.352 & nl 6 & $9 / 2$ & 10356.752 & $9 / 2$ & 27604.203 \\
\hline 5798.198 & 6 & $15 / 2$ & 11483.450 & $13 / 2$ & 28725.290 \\
\hline 5812.204 & 8 & $15 / 2$ & 8765.556 & $13 / 2$ & 25965.963 \\
\hline 5815.525 & 25 & $15 / 2$ & 29412.690 & $13 / 2$ & 12222.100 \\
\hline 5817.908 & nl 16 & $15 / 2$ & 13439.023 & $15 / 2$ & 30622.592 \\
\hline 5818.866 & nl 13 & $13 / 2$ & 9464.455 & $13 / 2$ & 26645.170 \\
\hline 5820.126 & nl 9 & $9 / 2$ & 12519.718 & $11 / 2$ & 29696.709 \\
\hline 5823.152 & 8 & $7 / 2$ & 28104.690 & $9 / 2$ & 10936.670 \\
\hline 5825.121 & nl 14 & $7 / 2$ & 10194.780 & $7 / 2$ & 27357.054 \\
\hline 5827.462 & nl 30 & $11 / 2$ & 6892.949 & $11 / 2$ & 24048.320 \\
\hline 5837.561 & nl 10 & $9 / 2$ & 11549.619 & $9 / 2$ & 28675.308 \\
\hline
\end{tabular}

$\mathrm{nl}$... new line, ${ }^{a}$ previously unknown energy level 
Table 1 - Continued

\begin{tabular}{|c|c|c|c|c|c|}
\hline \multirow[b]{2}{*}{$\lambda / \AA$} & \multirow[b]{2}{*}{ SNR } & \multicolumn{2}{|r|}{ Even } & \multicolumn{2}{|r|}{ Odd } \\
\hline & & J-value & Level Energy $/ \mathrm{cm}^{-1}$ & J-value & Level Energy $/ \mathrm{cm}^{-1}$ \\
\hline 5843.479 & 53 & $9 / 2$ & 8320.255 & $11 / 2$ & 25428.607 \\
\hline 5846.782 & nl 10 & $13 / 2$ & 10423.669 & $11 / 2$ & 27522.363 \\
\hline 5855.199 & nl 12 & $7 / 2$ & 15634.176 & $5 / 2$ & 32708.095 \\
\hline 5855.368 & nl 19 & $11 / 2$ & 19920.355 & $13 / 2$ & 2846.744 \\
\hline 5864.496 & nl 8 & $13 / 2$ & 12041.668 & $13 / 2$ & 29088.699 \\
\hline 5866.071 & nl 24 & $7 / 2$ & 17042.405 & $9 / 2$ & 0 \\
\hline 5875.487 & 28 & $11 / 2$ & 10904.049 & $11 / 2$ & 27919.207 \\
\hline 5879.378 & nl 8 & $7 / 2$ & 9918.205 & $5 / 2$ & 26922.093 \\
\hline 5884.989 & 8 & $13 / 2$ & 12041.668 & $11 / 2$ & 29029.547 \\
\hline 5893.492 & 234 & $11 / 2$ & 6714.199 & $13 / 2$ & 23677.365 \\
\hline 5896.218 & 17 & $9 / 2$ & 25205.463 & $9 / 2$ & 8250.170 \\
\hline 5897.409 & nl 11 & $7 / 2$ & 7617.455 & $9 / 2$ & 24569.356 \\
\hline 5902.991 & 40 & $11 / 2$ & 16935.880 & $9 / 2$ & 0 \\
\hline 5904.524 & nl 6 & $17 / 2$ & 12736.636 & $17 / 2$ & 29668.103 \\
\hline 5906.326 & 15 & $9 / 2$ & 5822.890 & $11 / 2$ & $22749.175^{a}$ \\
\hline 5906.519 & 14 & $13 / 2$ & 9464.455 & $13 / 2$ & 26390.208 \\
\hline 5907.249 & 112 & $9 / 2$ & 18300.341 & $11 / 2$ & 1376.605 \\
\hline 5924.633 & 25 & $13 / 2$ & 10423.669 & $13 / 2$ & 27297.680 \\
\hline 5929.330 & 80 & $11 / 2$ & 18237.240 & $11 / 2$ & 1376.605 \\
\hline 5934.451 & 17 & $13 / 2$ & 15772.560 & $13 / 2$ & 32618.845 \\
\hline 5936.246 & nl 58 & $9 / 2$ & 8029.290 & $11 / 2$ & 24870.318 \\
\hline 5938.827 & 24 & $11 / 2$ & 6313.239 & $13 / 2$ & 23146.917 \\
\hline 5938.228 & nl 11 & $11 / 2$ & 16835.400 & $9 / 2$ & 0 \\
\hline 5940.178 & 34 & $13 / 2$ & 29051.942 & $13 / 2$ & 12222.100 \\
\hline 5940.449 & nl 22 & $13 / 2$ & 28643.769 & $15 / 2$ & 11814.660 \\
\hline 5949.765 & nl 171 & $17 / 2$ & 27523.120 & $17 / 2$ & 10720.400 \\
\hline 5954.881 & 36 & $13 / 2$ & 10423.669 & $15 / 2$ & 27211.960 \\
\hline 5957.238 & 11 & $13 / 2$ & 12118.054 & $15 / 2$ & 28899.719 \\
\hline 5960.933 & 110 & $7 / 2$ & 7617.455 & $5 / 2$ & $24388.702^{a}$ \\
\hline 5970.186 & 17 & $15 / 2$ & 10466.704 & $15 / 2$ & 27211.960 \\
\hline 5971.086 & nl 18 & $7 / 2$ & 11869.331 & $9 / 2$ & 28612.095 \\
\hline 5975.644 & 16 & $9 / 2$ & 27384.059 & $7 / 2$ & 10654.110 \\
\hline 5977.377 & nl 8 & $11 / 2$ & 10904.049 & $13 / 2$ & 27629.152 \\
\hline 5985.748 & nl 31 & $7 / 2$ & 28104.689 & $7 / 2$ & 11403.067 \\
\hline 5987.819 & nl 94 & $9 / 2$ & 8320.255 & $11 / 2$ & 25016.201 \\
\hline 5993.095 & 16 & $7 / 2$ & 28731.220 & $9 / 2$ & 12049.950 \\
\hline 5994.418 & nl 82 & $9 / 2$ & 18054.202 & $11 / 2$ & 1376.605 \\
\hline 6011.218 & 35 & $9 / 2$ & 8320.255 & $9 / 2$ & 24951.215 \\
\hline 6014.607 & 10 & $13 / 2$ & 10423.669 & $15 / 2$ & 27045.255 \\
\hline 6021.988 & 22 & $9 / 2$ & 8320.255 & $11 / 2$ & 24921.466 \\
\hline 6023.642 & 68 & $15 / 2$ & 27265.622 & $15 / 2$ & 10668.960 \\
\hline 6030.234 & 54 & $15 / 2$ & 28342.772 & $17 / 2$ & 11764.250 \\
\hline 6035.413 & 21 & $11 / 2$ & 6313.239 & $9 / 2$ & $22877.542^{a}$ \\
\hline 6047.884 & 18 & $7 / 2$ & 12049.480 & $9 / 2$ & 28579.611 \\
\hline 6054.231 & 22 & $5 / 2$ & 6451.823 & $7 / 2$ & $22964.630^{a}$ \\
\hline 6061.992 & 70 & $9 / 2$ & 27145.754 & $7 / 2$ & 10654.110 \\
\hline 6068.436 & nl 8 & $13 / 2$ & 11562.777 & $13 / 2$ & 28036.928 \\
\hline
\end{tabular}

$\mathrm{nl}$... new line, ${ }^{a}$ previously unknown energy level 
Table 1 - Continued

\begin{tabular}{|c|c|c|c|c|c|}
\hline \multirow[b]{2}{*}{$\lambda / \AA$} & \multirow[b]{2}{*}{ SNR } & \multicolumn{2}{|r|}{ Even } & \multicolumn{2}{|r|}{ Odd } \\
\hline & & J-value & Level Energy $/ \mathrm{cm}^{-1}$ & J-value & Level Energy $/ \mathrm{cm}^{-1}$ \\
\hline 6069.197 & nl 20 & $7 / 2$ & 8013.104 & $9 / 2$ & $24485.187^{a}$ \\
\hline 6072.077 & 39 & $15 / 2$ & 27133.216 & $15 / 2$ & 10668.960 \\
\hline 6074.285 & 18 & $17 / 2$ & 10531.966 & $17 / 2$ & 26990.251 \\
\hline 6074.993 & 134 & $9 / 2$ & 24706.499 & $9 / 2$ & 8250.170 \\
\hline 6076.144 & nl 12 & $7 / 2$ & 28731.220 & $7 / 2$ & 12277.947 \\
\hline 6083.735 & 78 & $11 / 2$ & 6714.199 & $13 / 2$ & 23146.917 \\
\hline 6085.810 & 142 & $9 / 2$ & 24677.280 & $9 / 2$ & 8250.170 \\
\hline 6094.119 & 31 & $13 / 2$ & 25138.184 & $13 / 2$ & 8733.450 \\
\hline 6100.703 & 35 & $7 / 2$ & 16387.020 & $9 / 2$ & 0 \\
\hline 6105.825 & 18 & $5 / 2$ & 9710.615 & $7 / 2$ & 26083.919 \\
\hline 6107.014 & 90 & $9 / 2$ & 25205.463 & $11 / 2$ & 8835.380 \\
\hline 6118.016 & 50 & $11 / 2$ & 24590.800 & $9 / 2$ & 8250.170 \\
\hline 6124.960 & 46 & $9 / 2$ & 16322.152 & $9 / 2$ & 0 \\
\hline 6136.690 & 24 & $11 / 2$ & 9675.044 & $13 / 2$ & 25965.963 \\
\hline 6138.092 & 9 & $15 / 2$ & 30283.139 & $13 / 2$ & 13996.091 \\
\hline 6149.670 & 20 & $7 / 2$ & 7617.455 & $5 / 2$ & 23874.021 \\
\hline 6153.001 & nl 15 & $11 / 2$ & 6313.239 & $9 / 2$ & $22560.965^{a}$ \\
\hline 6161.073 & 34 & $9 / 2$ & 8643.839 & $11 / 2$ & 24870.318 \\
\hline 6179.837 & 28 & $13 / 2$ & 10470.344 & $15 / 2$ & 26647.525 \\
\hline 6191.936 & 10 & $13 / 2$ & 6603.606 & $11 / 2$ & $22749.175^{a}$ \\
\hline 6222.780 & 9 & $13 / 2$ & 10266.516 & $15 / 2$ & 26332.055 \\
\hline 6223.887 & nl 6 & $9 / 2$ & 28883.770 & $9 / 2$ & 12821.083 \\
\hline 6232.579 & 14 & $13 / 2$ & 7630.147 & $11 / 2$ & 23670.431 \\
\hline 6236.807 & 102 & $19 / 2$ & 29309.852 & $17 / 2$ & 13280.440 \\
\hline 6238.372 & 95 & $7 / 2$ & 6535.587 & $9 / 2$ & $22560.965^{a}$ \\
\hline 6239.883 & nl 8 & $11 / 2$ & 9268.741 & $9 / 2$ & 25290.248 \\
\hline 6242.330 & nl 9 & $9 / 2$ & 10356.752 & $7 / 2$ & 26371.986 \\
\hline 6246.546 & nl 9 & $11 / 2$ & 28054.356 & $9 / 2$ & 12049.950 \\
\hline 6251.682 & 10 & $11 / 2$ & 30217.484 & $11 / 2$ & 14226.248 \\
\hline 6270.364 & 46 & $15 / 2$ & 27689.962 & $13 / 2$ & 11746.340 \\
\hline 6280.999 & 54 & $11 / 2$ & 27662.982 & $13 / 2$ & 11746.340 \\
\hline 6292.876 & 22 & $11 / 2$ & 28764.269 & $11 / 2$ & 12877.670 \\
\hline 6297.347 & 23 & $15 / 2$ & 27689.962 & $15 / 2$ & 11814.660 \\
\hline 6298.408 & nl 12 & $11 / 2$ & 27195.110 & $11 / 2$ & 11322.420 \\
\hline 6299.531 & 28 & $11 / 2$ & 4866.530 & $9 / 2$ & $20736.338^{a}$ \\
\hline 6311.952 & 4 & $13 / 2$ & 6603.606 & $15 / 2$ & $22442.185^{a}$ \\
\hline 6312.170 & nl 4 & $11 / 2$ & 13250.690 & $13 / 2$ & 29088.699 \\
\hline 6334.990 & 35 & $15 / 2$ & $26191.740^{a}$ & $13 / 2$ & 10410.760 \\
\hline 6337.797 & 17 & $7 / 2$ & 26428.100 & $7 / 2$ & 10654.110 \\
\hline 6356.666 & 10 & $9 / 2$ & 10356.752 & $7 / 2$ & 26083.919 \\
\hline 6367.890 & 10 & $13 / 2$ & 10266.510 & $13 / 2$ & 25965.900 \\
\hline 6372.371 & nl 16 & $5 / 2$ & 9710.615 & $7 / 2$ & 25399.020 \\
\hline 6384.451 & 14 & $9 / 2$ & 27936.663 & $7 / 2$ & 12277.947 \\
\hline 6388.559 & 35 & $15 / 2$ & 27394.976 & $13 / 2$ & 11746.340 \\
\hline 6389.825 & 10 & $11 / 2$ & 12658.416 & $13 / 2$ & 28303.969 \\
\hline 6391.011 & 17 & $13 / 2$ & 6603.606 & $11 / 2$ & 22246.255 \\
\hline 6416.572 & 15 & $15 / 2$ & 27394.976 & $15 / 2$ & 11814.660 \\
\hline
\end{tabular}

$\mathrm{nl}$... new line, ${ }^{a}$ previously unknown energy level 
Table 1 - Continued

\begin{tabular}{|c|c|c|c|c|c|}
\hline \multirow[b]{2}{*}{$\lambda / \AA$} & \multirow[b]{2}{*}{ SNR } & \multicolumn{2}{|r|}{ Even } & \multicolumn{2}{|r|}{ Odd } \\
\hline & & J-value & Level Energy $/ \mathrm{cm}^{-1}$ & J-value & Level Energy $/ \mathrm{cm}^{-1}$ \\
\hline 6417.480 & nl 15 & $11 / 2$ & 11944.223 & $11 / 2$ & 27522.363 \\
\hline 6425.462 & 46 & $13 / 2$ & 6603.606 & $15 / 2$ & 22162.410 \\
\hline 6438.160 & nl 7 & $13 / 2$ & $13659 / 247$ & $15 / 2$ & 29182.662 \\
\hline 6440.364 & 53 & $13 / 2$ & 13146.599 & $11 / 2$ & 28669.174 \\
\hline 6440.366 & 54 & $15 / 2$ & $26191.740^{a}$ & $15 / 2$ & 10668.960 \\
\hline 6441.818 & 56 & $15 / 2$ & 27265.622 & $13 / 2$ & 11746.340 \\
\hline 6442.859 & 14 & $13 / 2$ & 7630.147 & $13 / 2$ & 23146.917 \\
\hline 6469.039 & nl 10 & $13 / 2$ & 25138.184 & $13 / 2$ & 9684.190 \\
\hline 6472.773 & 34 & $7 / 2$ & 26586.550 & $5 / 2$ & 11141.581 \\
\hline 6479.760 & nl 6 & $11 / 2$ & 28764.269 & $13 / 2$ & 13335.895 \\
\hline 6485.370 & nl 18 & $9 / 2$ & 27145.754 & $9 / 2$ & 11730.600 \\
\hline 6486.560 & 650 & $17 / 2$ & 25059.089 & $15 / 2$ & 9646.850 \\
\hline 6516.343 & nl 9 & $9 / 2$ & 19871.160 & $9 / 2$ & 35213.021 \\
\hline 6523.982 & 20 & $9 / 2$ & 8029.290 & $7 / 2$ & $23353.116^{a}$ \\
\hline $6539 / 224$ & 61 & $19 / 2$ & 30098.998 & $19 / 2$ & 14799.880 \\
\hline 6537.471 & 9 & $11 / 2$ & 10904.049 & $9 / 2$ & 26196.253 \\
\hline 6539.925 & 22 & $7 / 2$ & 26428.100 & $5 / 2$ & 11141.581 \\
\hline 6559.597 & 10 & $13 / 2$ & 27444.925 & $11 / 2$ & 12204.304 \\
\hline 6571.034 & nl 32 & $15 / 2$ & 8363.916 & $15 / 2$ & $23578.018^{a}$ \\
\hline 6576.070 & 9 & $11 / 2$ & 26524.890 & $11 / 2$ & 11322.420 \\
\hline 6577.508 & nl 11 & $11 / 2$ & 11282.880 & $9 / 2$ & 26482.007 \\
\hline 6581.885 & 7 & $5 / 2$ & 9710.615 & $7 / 2$ & 24899.636 \\
\hline 6583.537 & nl 6 & $11 / 2$ & 9483.533 & $13 / 2$ & 24668.738 \\
\hline 6616.025 & 196 & $7 / 2$ & 15110.661 & $9 / 2$ & 0 \\
\hline 6617.063 & nl 4 & $17 / 2$ & 12736.636 & $17 / 2$ & 27844.904 \\
\hline 6617.336 & 51 & $15 / 2$ & $24759 / 250$ & $15 / 2$ & 9646.850 \\
\hline 6630.181 & 15 & $9 / 2$ & 9105.035 & $11 / 2$ & $24183.415^{a}$ \\
\hline 6632.770 & nl 4 & $15 / 2$ & 29412.685 & $17 / 2$ & 14340.210 \\
\hline 6636.333 & nl 5 & $13 / 2$ & 9464.455 & $15 / 2$ & 24528.854 \\
\hline 6650.257 & nl 3 & $9 / 2$ & 8320.255 & $7 / 2$ & $23353.116^{a}$ \\
\hline $6709 / 237$ & 10 & $15 / 2$ & 27133.260 & $13 / 2$ & 12222.100 \\
\hline 6725.360 & 85 & $5 / 2$ & 6451.823 & $7 / 2$ & 21316.810 \\
\hline 6749.209 & 50 & $15 / 2$ & 8765.556 & $15 / 2$ & $23578.018^{a}$ \\
\hline 6763.722 & 5 & $17 / 2$ & 14028.722 & $19 / 2$ & 28809.400 \\
\hline 6765.297 & 12 & $15 / 2$ & 28342.772 & $15 / 2$ & 13565.520 \\
\hline 6686.434 & nl 10 & $7 / 2$ & 8013.104 & $7 / 2$ & $22964.630^{a}$ \\
\hline 6703.511 & 13 & $9 / 2$ & 5822.905 & $9 / 2$ & $20736.338^{a}$ \\
\hline 6744.932 & nl 14 & $11 / 2$ & 6892.949 & $11 / 2$ & $21714.802^{a}$ \\
\hline 6782.978 & nl 21 & $3 / 2$ & 9650.060 & $5 / 2$ & $24388.702^{a}$ \\
\hline 6788.184 & 21 & $13 / 2$ & 25138.184 & $13 / 2$ & 10410.760 \\
\hline 6797.865 & 34 & $13 / 2$ & 25138.184 & $11 / 2$ & 10431.720 \\
\hline 6799.1 & 37 & $11 / 2$ & 25135.518 & $11 / 2$ & 10431.720 \\
\hline 6808.396 & nl 5 & $7 / 2$ & 7617.455 & $7 / 2$ & $22301.145^{a}$ \\
\hline 6810.509 & 10 & $15 / 2$ & 9745.420 & $17 / 2$ & $24429 / 240$ \\
\hline 6810.994 & nl 28 & $5 / 2$ & 9710.640 & $5 / 2$ & $24388.702^{a}$ \\
\hline 6826.675 & nl 110 & $9 / 2$ & 8320.255 & $7 / 2$ & $22964.630^{a}$ \\
\hline 6833.879 & nl 9 & $9 / 2$ & 26032.009 & $7 / 2$ & 11403.067 \\
\hline
\end{tabular}

$\mathrm{nl}$... new line, ${ }^{a}$ previously unknown energy level 
Table 1 - Continued

\begin{tabular}{|c|c|c|c|c|c|}
\hline \multirow[b]{2}{*}{$\lambda / \AA$} & \multirow[b]{2}{*}{ SNR } & \multicolumn{2}{|r|}{ Even } & \multicolumn{2}{|r|}{ Odd } \\
\hline & & J-value & Level Energy $/ \mathrm{cm}^{-1}$ & J-value & Level Energy $/ \mathrm{cm}^{-1}$ \\
\hline 6845.305 & 58 & $5 / 2$ & 6451.823 & $5 / 2$ & $21056.348^{a}$ \\
\hline 6847.480 & 27 & $13 / 2$ & 10423.669 & $15 / 2$ & 25023.550 \\
\hline 6855.001 & nl 7 & $13 / 2$ & 9464.455 & $11 / 2$ & 24048.320 \\
\hline 6855.322 & 18 & $15 / 2$ & 17429.980 & $13 / 2$ & 2846.744 \\
\hline 6885.420 & 12 & $17 / 2$ & 9770.330 & $19 / 2$ & $24289.760^{a}$ \\
\hline 6889.913 & 24 & $19 / 2$ & 29309.852 & $19 / 2$ & 14799.880 \\
\hline 6893.023 & 36 & $7 / 2$ & 27883.218 & $9 / 2$ & 13379.780 \\
\hline 6904.230 & 8 & $15 / 2$ & 12250.534 & $13 / 2$ & 26730.415 \\
\hline 6930.502 & nl 8 & $5 / 2$ & 8737.448 & $7 / 2$ & 23162.497 \\
\hline 6931.411 & 65 & $11 / 2$ & 6313.239 & $9 / 2$ & $20736.338^{a}$ \\
\hline 6941.753 & 45 & $13 / 2$ & 17248.381 & $13 / 2$ & 2846.744 \\
\hline 6942.556 & 11 & $13 / 2$ & 10470.344 & $11 / 2$ & 24870.228 \\
\hline 6944.485 & nl 10 & $13 / 2$ & 15772.560 & $11 / 2$ & 1376.605 \\
\hline 6954.744 & nl 14 & $5 / 2$ & 6451.823 & $5 / 2$ & $20826.530^{a}$ \\
\hline 6995.508 & 52 & $7 / 2$ & 6535.587 & $5 / 2$ & $20826.530^{a}$ \\
\hline 6998.848 & 15 & $15 / 2$ & 27620.060 & $13 / 2$ & 13335.895 \\
\hline 7004.687 & 10 & $9 / 2$ & 13432.490 & $7 / 2$ & 27704.707 \\
\hline 7004.906 & nl 17 & $15 / 2$ & 17118.558 & $13 / 2$ & 2846.744 \\
\hline 7008.332 & 6 & $11 / 2$ & 22998.253 & $13 / 2$ & 8733.450 \\
\hline 7008.775 & 44 & $5 / 2$ & 6451.823 & $3 / 2$ & 20715.719 \\
\hline 7016.555 & 14 & $9 / 2$ & 9105.035 & $7 / 2$ & $23353.116^{a}$ \\
\hline 7026.094 & $\mathrm{nl} 10$ & $11 / 2$ & 6714.199 & $11 / 2$ & $20942.933^{a}$ \\
\hline 7037.560 & nl 16 & $7 / 2$ & 8013.104 & $9 / 2$ & 22218.655 \\
\hline 7038.110 & 6 & $9 / 2$ & 9105.035 & $11 / 2$ & 23309.476 \\
\hline 7039.939 & 23 & $7 / 2$ & 6535.587 & $9 / 2$ & $20736.338^{a}$ \\
\hline 7041.108 & nl 4 & $13 / 2$ & 10470.344 & $13 / 2$ & 24668.738 \\
\hline 7070.533 & 30 & $9 / 2$ & 14139.300 & $9 / 2$ & 0 \\
\hline 7076.126 & $\mathrm{nl} 4$ & $11 / 2$ & 9483.533 & $11 / 2$ & 23611.664 \\
\hline 7083.429 & 200 & $13 / 2$ & 9464.460 & $15 / 2$ & $23578.018^{a}$ \\
\hline 7091.629 & nl 7 & $5 / 2$ & 8737.448 & $7 / 2$ & 22834.790 \\
\hline 7094.691 & 22 & $5 / 2$ & 6451.823 & $7 / 2$ & 20542.983 \\
\hline 7097.968 & 26 & $13 / 2$ & 7630.147 & $11 / 2$ & $21714.802^{a}$ \\
\hline 7101.187 & nl 12 & $15 / 2$ & 8363.910 & $15 / 2$ & $22442.185^{a}$ \\
\hline 7115.484 & 18 & $11 / 2$ & 6892.949 & $11 / 2$ & $20942.933^{a}$ \\
\hline 7117.560 & 24 & $11 / 2$ & 16935.880 & $9 / 2$ & 30981.669 \\
\hline 7128.169 & nl 24 & $13 / 2$ & 7630.147 & $15 / 2$ & 21655.124 \\
\hline 7134.864 & nl 8 & $13 / 2$ & 7630.170 & $13 / 2$ & 21641.950 \\
\hline 7150.649 & 16 & $9 / 2$ & 8320.255 & $7 / 2$ & $22301.160^{a}$ \\
\hline 7163.745 & 15 & $17 / 2$ & 12736.670 & $17 / 2$ & $26692.000^{a}$ \\
\hline 7183.412 & nl 10 & $9 / 2$ & 8643.839 & $9 / 2$ & $22560.965^{a}$ \\
\hline 7216.421 & nl 4 & $13 / 2$ & 27213.988 & $11 / 2$ & 13360.529 \\
\hline 7221.674 & 36 & $11 / 2$ & 6892.949 & $9 / 2$ & $20736.338^{a}$ \\
\hline 7240.324 & nl 6 & $17 / 2$ & 9770.330 & $15 / 2$ & $23578.018^{a}$ \\
\hline 7245.184 & 21 & $15 / 2$ & 8363.916 & $15 / 2$ & 22162.410 \\
\hline 7255.175 & 380 & $13 / 2$ & 15156.070 & $11 / 2$ & 1376.605 \\
\hline 7265.574 & nl 5 & $13 / 2$ & 10423.680 & $11 / 2$ & $24183.415^{a}$ \\
\hline 7265.838 & 7 & $17 / 2$ & 18380.340 & $17 / 2$ & 32139.568 \\
\hline
\end{tabular}

$\mathrm{nl}$... new line, ${ }^{a}$ previously unknown energy level 
Table 1 - Continued

\begin{tabular}{|c|c|c|c|c|c|}
\hline \multirow[b]{2}{*}{$\lambda / \AA$} & \multirow[b]{2}{*}{ SNR } & \multicolumn{2}{|r|}{ Even } & \multicolumn{2}{|r|}{ Odd } \\
\hline & & J-value & Level Energy $/ \mathrm{cm}^{-1}$ & J-value & Level Energy $/ \mathrm{cm}^{-1}$ \\
\hline 7266.625 & 17 & $17 / 2$ & 10532.001 & $19 / 2$ & $24289.760^{a}$ \\
\hline 7278.726 & 110 & $11 / 2$ & 15111.480 & $11 / 2$ & 1376.605 \\
\hline 7280.314 & 35 & $11 / 2$ & 8829.078 & $9 / 2$ & $22560.965^{a}$ \\
\hline 7281.341 & $\mathrm{nl} 5$ & $9 / 2$ & 13974.730 & $7 / 2$ & 27704.707 \\
\hline 7302.256 & $\mathrm{nl} 5$ & $13 / 2$ & 7951.350 & $13 / 2$ & 21641.950 \\
\hline 7309.733 & nl 5 & $15 / 2$ & 8765.570 & $15 / 2$ & $22442.185^{a}$ \\
\hline 7316.682 & 13 & $11 / 2$ & 14505.090 & $11 / 2$ & 28168.714 \\
\hline 7319.268 & 23 & $7 / 2$ & 14045.708 & $7 / 2$ & 27704.707 \\
\hline 7320.071 & 33 & $9 / 2$ & 8643.839 & $9 / 2$ & $22301.160^{a}$ \\
\hline 7320.626 & nl 7 & $7 / 2$ & 7617.455 & $9 / 2$ & 21273.728 \\
\hline 7328.016 & 15 & $5 / 2$ & 9710.640 & $7 / 2$ & $23353.116^{a}$ \\
\hline 7335.136 & $\mathrm{nl} 2$ & $13 / 2$ & 24951.730 & $11 / 2$ & 11322.420 \\
\hline 7337.282 & nl 4 & $11 / 2$ & 6714.220 & $13 / 2$ & 20339.470 \\
\hline 7337.714 & 12 & $13 / 2$ & 13897.891 & $11 / 2$ & 27522.363 \\
\hline 7346.172 & nl 5 & $11 / 2$ & 9268.741 & $9 / 2$ & $22877.524^{a}$ \\
\hline 7352.265 & nl 5 & $15 / 2$ & 11483.442 & $13 / 2$ & 25080.951 \\
\hline 7354.833 & nl 3 & $13 / 2$ & 10266.516 & $11 / 2$ & 23859.275 \\
\hline 7364.208 & 15 & $13 / 2$ & 16422.207 & $13 / 2$ & 2846.744 \\
\hline 7379.937 & nl 5 & $11 / 2$ & 551.949 & $13 / 2$ & 20439.490 \\
\hline 7390.274 & $\mathrm{nl} 2$ & $15 / 2$ & 12804.483 & $15 / 2$ & 26332.055 \\
\hline 7393.802 & nl 6 & $9 / 2$ & 14897.770 & $11 / 2$ & 1376.620 \\
\hline 7404.778 & nl 2 & $11 / 2$ & 23185.233 & $13 / 2$ & 9684.190 \\
\hline 7428.425 & nl 3 & $11 / 2$ & 13250.690 & $13 / 2$ & 26708.776 \\
\hline 7436.633 & nl 12 & $13 / 2$ & 7951.338 & $11 / 2$ & $21399 / 281$ \\
\hline 7439.04 & 36 & $7 / 2$ & 7617.455 & $5 / 2$ & $21056.348^{a}$ \\
\hline 7440.860 & $\mathrm{nl} 4$ & $13 / 2$ & 10423.680 & $11 / 2$ & 23859.280 \\
\hline 7462.398 & $\mathrm{nl} 4$ & $15 / 2$ & 8765.570 & $15 / 2$ & 22162.410 \\
\hline 7463.978 & nl 9 & $11 / 2$ & 9483.533 & $9 / 2$ & $22877.524^{a}$ \\
\hline 7467.496 & 10 & $9 / 2$ & 14764.290 & $11 / 2$ & 1376.605 \\
\hline 7479.184 & nl 3 & $9 / 2$ & 10356.752 & $7 / 2$ & 23723.515 \\
\hline 7483.183 & nl 8 & $7 / 2$ & 9704.750 & $7 / 2$ & $23064.370^{a}$ \\
\hline 7498.888 & nl 7 & $9 / 2$ & 12746.082 & $7 / 2$ & 26077.608 \\
\hline 7509.508 & nl 8 & $13 / 2$ & 7630.147 & $11 / 2$ & $20942.933^{a}$ \\
\hline 7511.542 & nl 15 & $17 / 2$ & 11714.367 & $15 / 2$ & 25023.550 \\
\hline 7523.291 & 10 & $9 / 2$ & 10920.378 & $9 / 2$ & 24208.775 \\
\hline 7536.197 & nl 6 & $11 / 2$ & 9483.540 & $11 / 2$ & $22749.175^{a}$ \\
\hline 7539.478 & nl 10 & $7 / 2$ & 9704.750 & $7 / 2$ & $22964.630^{a}$ \\
\hline 7542.806 & 12 & $5 / 2$ & 9710.640 & $7 / 2$ & $22964.630^{a}$ \\
\hline 7555.046 & nl 8 & $13 / 2$ & 24554.990 & $11 / 2$ & 11322.420 \\
\hline 7568.468 & nl 15 & $7 / 2$ & 7617.455 & $5 / 2$ & $20826.530^{a}$ \\
\hline 7569.105 & nl 6 & $13 / 2$ & 16250.9 & $11 / 2$ & 29458.91 \\
\hline 7573.242 & nl 3 & $5 / 2$ & 8737.448 & $5 / 2$ & 21938.328 \\
\hline 7599.958 & 10 & $13 / 2$ & 10423.680 & $15 / 2$ & $23578.018^{a}$ \\
\hline 7602.328 & nl 2 & $9 / 2$ & 8320.255 & $11 / 2$ & 21470.498 \\
\hline 7603.604 & 20 & $15 / 2$ & 15994.810 & $13 / 2$ & 2846.744 \\
\hline 7606.547 & nl 1 & $15 / 2$ & 11483.442 & $15 / 2$ & 24626.392 \\
\hline 7609.254 & nl 17 & $19 / 2$ & 11151.490 & $19 / 2$ & $24289.760^{a}$ \\
\hline
\end{tabular}

$\mathrm{nl}$... new line, ${ }^{a}$ previously unknown energy level 
Table 1 - Continued

\begin{tabular}{|c|c|c|c|c|c|}
\hline \multirow[b]{2}{*}{$\lambda / \AA$} & \multirow[b]{2}{*}{ SNR } & \multicolumn{2}{|r|}{ Even } & \multicolumn{2}{|r|}{ Odd } \\
\hline & & J-value & Level Energy $/ \mathrm{cm}^{-1}$ & J-value & Level Energy $/ \mathrm{cm}^{-1}$ \\
\hline 7620.501 & nl 6 & $7 / 2$ & 7617.455 & $9 / 2$ & $20736.338^{a}$ \\
\hline 7636.327 & 40 & $9 / 2$ & 14468.310 & $11 / 2$ & 1376.605 \\
\hline 7651.767 & 36 & $19 / 2$ & 13626.720 & $17 / 2$ & $266920^{a}$ \\
\hline 7661.401 & 15 & $15 / 2$ & 17429.980 & $15 / 2$ & 4381.100 \\
\hline 7668.138 & 16 & $17 / 2$ & 11714.380 & $19 / 2$ & $24751.780^{a}$ \\
\hline 7684.518 & 18 & $11 / 2$ & 8829.078 & $9 / 2$ & 21838.677 \\
\hline 7713.015 & 30 & $17 / 2$ & 14028.722 & $17 / 2$ & 26990.251 \\
\hline 7714.329 & 15 & $7 / 2$ & 9918.170 & $9 / 2$ & $22877.524^{a}$ \\
\hline 7725.252 & nl 12 & $13 / 2$ & 10266.510 & $11 / 2$ & 23207.530 \\
\hline $7789 / 220$ & nl 6 & $17 / 2$ & 12736.636 & $17 / 2$ & 25579.110 \\
\hline 7790.889 & 5 & $13 / 2$ & 16626.890 & $11 / 2$ & 29458.910 \\
\hline 7802.166 & 14 & $7 / 2$ & 8013.104 & $5 / 2$ & $20826.530^{a}$ \\
\hline 7804.406 & 20 & $9 / 2$ & 14186.360 & $11 / 2$ & 1376.605 \\
\hline 7806.808 & nl 13 & $9 / 2$ & 10356.752 & $7 / 2$ & 23162.497 \\
\hline 7817.033 & nl 3 & $13 / 2$ & 13897.891 & $11 / 2$ & 26686.944 \\
\hline 7842.577 & nl 6 & $13 / 2$ & 24951.730 & $11 / 2$ & 12204.304 \\
\hline 7857.475 & nl 6 & $7 / 2$ & 8013.104 & $9 / 2$ & $20736.338^{a}$ \\
\hline 7866.150 & nl 5 & $13 / 2$ & 11462.912 & $15 / 2$ & 24172.223 \\
\hline 7873.840 & nl 5 & $15 / 2$ & 9745.391 & $15 / 2$ & $22442.185^{a}$ \\
\hline 7884.135 & 17 & $15 / 2$ & 10466.704 & $13 / 2$ & 23146.917 \\
\hline 7889.311 & 60 & $17 / 2$ & 9770.330 & $15 / 2$ & $22442.185^{a}$ \\
\hline 7910.501 & $\mathrm{nl} 5$ & $13 / 2$ & 13146.599 & $11 / 2$ & $25789 / 252$ \\
\hline 7912.997 & 34 & $11 / 2$ & 15111.480 & $9 / 2$ & 27745.287 \\
\hline 7919.993 & 10 & $11 / 2$ & 15469.560 & $13 / 2$ & 2846.744 \\
\hline 7929.466 & nl 2 & $11 / 2$ & 14760.320 & $9 / 2$ & 27368.267 \\
\hline 7940.172 & 29 & $5 / 2$ & 15113.984 & $7 / 2$ & 27704.707 \\
\hline 7965.293 & nl 9 & $5 / 2$ & 6451.823 & $7 / 2$ & $19002.837^{a}$ \\
\hline 7999.185 & nl 3 & $13 / 2$ & 10470.344 & $15 / 2$ & 22968.185 \\
\hline 8018.809 & 17 & $7 / 2$ & 6535.587 & $7 / 2$ & $19002.837^{a}$ \\
\hline 8027.119 & 8 & $7 / 2$ & 8013.108 & $5 / 2$ & $20467.450^{a}$ \\
\hline 8062.49 & 6 & $15 / 2$ & 16451.350 & $13 / 2$ & 28851.022 \\
\hline 8069.405 & nl 5 & $17 / 2$ & 14302.923 & $17 / 2$ & $26692.000^{a}$ \\
\hline 8086.784 & nl 3 & $11 / 2$ & 9268.741 & $9 / 2$ & 21631.198 \\
\hline 8111.028 & 20 & $13 / 2$ & 10423.680 & $11 / 2$ & $22749.175^{a}$ \\
\hline 8139.924 & nl 4 & $17 / 2$ & 11714.367 & $15 / 2$ & 23996.130 \\
\hline 8160.791 & 112 & $13 / 2$ & 9464.460 & $11 / 2$ & $21714.802^{a}$ \\
\hline 8170.833 & 12 & $5 / 2$ & 10828.988 & $7 / 2$ & $23064.370^{a}$ \\
\hline 8174.676 & 11 & $13 / 2$ & 11462.912 & $13 / 2$ & 23692.444 \\
\hline 8210.847 & 12 & $13 / 2$ & 10266.510 & $15 / 2$ & $22442.185^{a}$ \\
\hline 8218.971 & 5 & $11 / 2$ & 9675.044 & $9 / 2$ & 21838.677 \\
\hline 8222.482 & nl 3 & $11 / 2$ & 9483.533 & $13 / 2$ & 21641.964 \\
\hline 8233.786 & 10 & $13 / 2$ & 12041.670 & $11 / 2$ & $24183.415^{a}$ \\
\hline 8238.524 & 45 & $11 / 2$ & 14981.510 & $13 / 2$ & 2846.744 \\
\hline 8257.844 & nl 12 & $7 / 2$ & 10194.740 & $7 / 2$ & $22301.145^{a}$ \\
\hline 8276.642 & nl 11 & $7 / 2$ & 11274.180 & $7 / 2$ & $23353.116^{a}$ \\
\hline 8301.271 & 12 & $7 / 2$ & 8013.104 & $5 / 2$ & 20056.146 \\
\hline 8320.561 & 36 & $17 / 2$ & 12736.670 & $19 / 2$ & $24751.780^{a}$ \\
\hline
\end{tabular}

$\mathrm{nl}$... new line, ${ }^{a}$ previously unknown energy level 
Table 1 - Continued

\begin{tabular}{|c|c|c|c|c|c|}
\hline \multirow[b]{2}{*}{$\lambda / \AA$} & \multirow[b]{2}{*}{ SNR } & \multicolumn{2}{|r|}{ Even } & \multicolumn{2}{|r|}{ Odd } \\
\hline & & J-value & Level Energy $/ \mathrm{cm}^{-1}$ & J-value & Level Energy $/ \mathrm{cm}^{-1}$ \\
\hline 8327.581 & nl 4 & $11 / 2$ & 9268.750 & $9 / 2$ & 21273.710 \\
\hline 8329.675 & 5 & $9 / 2$ & 14764.290 & $11 / 2$ & 26766.200 \\
\hline 8333.738 & nl 1 & $9 / 2$ & 13432.490 & $11 / 2$ & 25428.607 \\
\hline 8349.501 & nl 5 & $11 / 2$ & 10904.070 & $9 / 2$ & $22877.524^{a}$ \\
\hline 8376.978 & nl 35 & $15 / 2$ & 14780.939 & $13 / 2$ & 2846.744 \\
\hline 8391.486 & 10 & $11 / 2$ & 14760.320 & $13 / 2$ & 2846.744 \\
\hline 8393.847 & 10 & $17 / 2$ & 10532.001 & $15 / 2$ & $22442.185^{a}$ \\
\hline 8395.932 & 65 & $11 / 2$ & 8829.078 & $9 / 2$ & $20736.338^{a}$ \\
\hline 8415.219 & nl 11 & $9 / 2$ & 11184.410 & $7 / 2$ & $23064.370^{a}$ \\
\hline 8432.191 & nl 2 & $15 / 2$ & 15819.324 & $15 / 2$ & 27675.355 \\
\hline 8453.074 & nl 6 & $11 / 2$ & 12658.427 & $9 / 2$ & $24485.187^{a}$ \\
\hline 8488.317 & nl 3 & $3 / 2$ & 14420.003 & $5 / 2$ & 26197.8 \\
\hline 8526.492 & nl 3 & $9 / 2$ & 13605.68 & $9 / 2$ & 25330.615 \\
\hline 8539.710 & nl 5 & $5 / 2$ & 11646.260 & $7 / 2$ & $23353.116^{a}$ \\
\hline 8549.691 & nl13 & $9 / 2$ & 11184.410 & $9 / 2$ & $22877.542^{a}$ \\
\hline 8565.573 & nl 3 & $9 / 2$ & 8643.830 & $11 / 2$ & 20315.280 \\
\hline 8567.514 & 20 & $7 / 2$ & 11668.787 & $9 / 2$ & 0 \\
\hline 8571.418 & 12 & $15 / 2$ & 11483.442 & $13 / 2$ & 23146.917 \\
\hline 8575.204 & 6 & $11 / 2$ & 14505.090 & $13 / 2$ & 2846.744 \\
\hline 8588.806 & nl 3 & $9 / 2$ & 11713.220 & $7 / 2$ & $23353.116^{a}$ \\
\hline 8592.744 & nl 6 & $13 / 2$ & 13146.599 & $13 / 2$ & 24781.131 \\
\hline $8599 / 241$ & nl 3 & $13 / 2$ & 9464.460 & $13 / 2$ & 21096.530 \\
\hline 8614.061 & $\mathrm{nl} 2$ & $9 / 2$ & 8643.839 & $11 / 2$ & 20249.580 \\
\hline 8615.883 & nl 9 & $7 / 2$ & 11274.244 & $9 / 2$ & $22877.524^{a}$ \\
\hline 8624.300 & nl 15 & $7 / 2$ & 11472.423 & $7 / 2$ & $23064.370^{a}$ \\
\hline 8624.877 & nl 2 & $13 / 2$ & 24951.730 & $11 / 2$ & 13360.529 \\
\hline 8644.635 & 10 & $15 / 2$ & 13439.023 & $13 / 2$ & 25003.715 \\
\hline 8653.316 & nl 15 & $17 / 2$ & 12736.670 & $19 / 2$ & $24289.760^{a}$ \\
\hline 8682.117 & nl 5 & $9 / 2$ & 11549.614 & $7 / 2$ & $23064.370^{a}$ \\
\hline 8705.521 & nl 11 & $7 / 2$ & 11869.331 & $7 / 2$ & $23353.116^{a}$ \\
\hline 8709.564 & 34 & $13 / 2$ & 9464.460 & $11 / 2$ & $20942.933^{a}$ \\
\hline 8717.830 & nl 8 & $11 / 2$ & 9268.741 & $9 / 2$ & $20736.338^{a}$ \\
\hline 8718.817 & nl 5 & $11 / 2$ & 11282.870 & $11 / 2$ & $22749.175^{a}$ \\
\hline 8724.067 & nl 9 & $11 / 2$ & 9483.540 & $11 / 2$ & $20942.933^{a}$ \\
\hline 8764.630 & nl 22 & $3 / 2$ & 9650.060 & $5 / 2$ & $21056.348^{a}$ \\
\hline 8775.504 & 10 & $9 / 2$ & 13974.730 & $11 / 2$ & 25366.965 \\
\hline 8801.914 & 42 & $9 / 2$ & 10356.710 & $11 / 2$ & $21714.802^{a}$ \\
\hline 8811.465 & 27 & $5 / 2$ & 9710.640 & $5 / 2$ & $21056.348^{a}$ \\
\hline 8822.442 & 10 & $11 / 2$ & 14178.380 & $13 / 2$ & 2846.744 \\
\hline 8825.333 & 10 & $9 / 2$ & 11549.614 & $9 / 2$ & $22877.524^{a}$ \\
\hline 8847.259 & 5 & $11 / 2$ & 10904.070 & $11 / 2$ & 22203.860 \\
\hline 8859.101 & 13 & $17 / 2$ & 15665.829 & $15 / 2$ & 4381.120 \\
\hline 8881.379 & 18 & $13 / 2$ & 11462.858 & $13 / 2$ & 22719.340 \\
\hline 8918.421 & nl 10 & $11 / 2$ & 13376.992 & $11 / 2$ & 24586.670 \\
\hline 8962.403 & 20 & $3 / 2$ & 11361.817 & $1 / 2$ & $22516.490^{a}$ \\
\hline 8930.529 & 17 & $13 / 2$ & 24554.99 & $11 / 2$ & 13360.529 \\
\hline 8942.410 & 16 & $3 / 2$ & 11361.817 & $5 / 2$ & 22541.342 \\
\hline
\end{tabular}

$\mathrm{nl}$... new line, ${ }^{a}$ previously unknown energy level 
Table 1 - Continued

\begin{tabular}{|c|c|c|c|c|c|}
\hline \multirow[b]{2}{*}{$\lambda / \AA$} & \multirow[b]{2}{*}{ SNR } & \multicolumn{2}{|r|}{ Even } & \multicolumn{2}{|r|}{ Odd } \\
\hline & & J-value & Level Energy $/ \mathrm{cm}^{-1}$ & J-value & Level Energy $/ \mathrm{cm}^{-1}$ \\
\hline 8944.852 & 10 & $3 / 2$ & 9650.060 & $5 / 2$ & $20826.530^{a}$ \\
\hline 8954.679 & nl 25 & $9 / 2$ & 11713.249 & $9 / 2$ & $22877.524^{a}$ \\
\hline 8965.404 & 6 & $9 / 2$ & 14139.300 & $9 / 2$ & 25290.248 \\
\hline 8975.694 & nl 50 & $7 / 2$ & 9918.170 & $5 / 2$ & $21056.348^{a}$ \\
\hline 8986.243 & 95 & $19 / 2$ & 13626.720 & $19 / 2$ & $24751.780^{a}$ \\
\hline 8993.509 & 67 & $9 / 2$ & 19196.472 & $11 / 2$ & 8080.440 \\
\hline 9015.841 & nl 6 & $7 / 2$ & 11472.423 & $9 / 2$ & $22560.965^{a}$ \\
\hline 9016.229 & nl 6 & $3 / 2$ & 12701.269 & $3 / 2$ & 23789.203 \\
\hline 9019.598 & nl 3 & $5 / 2$ & 12078.583 & $7 / 2$ & 23162.497 \\
\hline 9058.096 & 28 & $13 / 2$ & 13146.600 & $11 / 2$ & $24183.415^{a}$ \\
\hline 9062.401 & nl 6 & $7 / 2$ & 9704.750 & $9 / 2$ & $20736.338^{a}$ \\
\hline 9066.240 & nl 50 & $7 / 2$ & 11274.180 & $7 / 2$ & $22301.145^{a}$ \\
\hline 9074.106 & nl 2 & $11 / 2$ & 23067.290 & $9 / 2$ & 12049.950 \\
\hline 9076.121 & nl 11 & $7 / 2$ & 12049.480 & $7 / 2$ & $23064.370^{a}$ \\
\hline 9078.497 & 8 & $11 / 2$ & 12658.416 & $11 / 2$ & 23670.431 \\
\hline 9079.042 & nl 7 & $9 / 2$ & 11549.614 & $9 / 2$ & $22560.965^{a}$ \\
\hline 9096.905 & 26 & $7 / 2$ & 8013.104 & $7 / 2$ & $19002.837^{a}$ \\
\hline 9110.821 & nl 13 & $7 / 2$ & 13415.769 & $5 / 2$ & $24388.702^{a}$ \\
\hline 9117.502 & 7 & $13 / 2$ & 14470.060 & $13 / 2$ & 25434.959 \\
\hline 9132.968 & 25 & $9 / 2$ & 19196.472 & $9 / 2$ & 8250.170 \\
\hline 9141.072 & nl 7 & $17 / 2$ & 22700.890 & $17 / 2$ & 11764.250 \\
\hline 9143.853 & $\mathrm{nl} 10$ & $11 / 2$ & 11944.223 & $9 / 2$ & $22877.524^{a}$ \\
\hline 9144.025 & 53 & $5 / 2$ & 9710.615 & $3 / 2$ & 20643.800 \\
\hline 9164.795 & nl 13 & $7 / 2$ & 9918.170 & $5 / 2$ & $20826.530^{a}$ \\
\hline 9174.839 & nl 4 & $15 / 2$ & 12250.534 & $13 / 2$ & 23146.917 \\
\hline 9176.122 & 27 & $7 / 2$ & 13590.350 & $9 / 2$ & $24485.187^{a}$ \\
\hline 9183.414 & nl 2 & $17 / 2$ & 22700.890 & $15 / 2$ & 11814.660 \\
\hline 9189.156 & 6 & $13 / 2$ & 11562.790 & $15 / 2$ & $22442.185^{a}$ \\
\hline 9204.247 & nl 13 & $7 / 2$ & 10194.740 & $5 / 2$ & $21056.348^{a}$ \\
\hline 9208.296 & nl 3 & $9 / 2$ & 8029.29 & $9 / 2$ & 18886.085 \\
\hline 9216.883 & nl 9 & $19 / 2$ & 15845.359 & $17 / 2$ & $26692.000^{a}$ \\
\hline 9230.499 & nl 21 & $13 / 2$ & 10470.344 & $11 / 2$ & 21301.023 \\
\hline 9232.162 & nl 9 & $7 / 2$ & 11572.350 & $7 / 2$ & $22301.160^{a}$ \\
\hline 9241.772 & 7 & $3 / 2$ & 9650.06 & $5 / 2$ & $20467.450^{a}$ \\
\hline 9247.458 & 15 & $9 / 2$ & 28936.494 & $9 / 2$ & 18125.523 \\
\hline 9248.361 & 10 & $17 / 2$ & 14028.722 & $15 / 2$ & 24838.484 \\
\hline 9283.717 & nl 10 & $7 / 2$ & 10194.740 & $7 / 2$ & 20963.330 \\
\hline 9293.861 & 50 & $5 / 2$ & 9710.640 & $5 / 2$ & $20467.450^{a}$ \\
\hline 9294.657 & 25 & $3 / 2$ & 13033.479 & $3 / 2$ & 23789.203 \\
\hline 9298.448 & 25 & $9 / 2$ & 11549.614 & $7 / 2$ & $22301.160^{a}$ \\
\hline 9307.186 & 11 & $13 / 2$ & 12118.060 & $11 / 2$ & 1376.620 \\
\hline 9319.006 & 50 & $17 / 2$ & 11714.367 & $15 / 2$ & $22442.185^{a}$ \\
\hline 9336.680 & 10 & $13 / 2$ & 12041.668 & $11 / 2$ & $22749.175^{a}$ \\
\hline 9337.905 & 6 & $5 / 2$ & 12647.027 & $7 / 2$ & $23353.116^{a}$ \\
\hline 9374.581 & 10 & $11 / 2$ & 15238.140 & $13 / 2$ & 25902.360 \\
\hline 9385.756 & 30 & $1 / 2$ & 11865.001 & $1 / 2$ & $22516.490^{a}$ \\
\hline 9403.13 & nl 5 & $7 / 2$ & 10194.740 & $5 / 2$ & $20826.530^{a}$ \\
\hline
\end{tabular}

$\mathrm{nl}$... new line, ${ }^{a}$ previously unknown energy level 
Table 1 - Continued

\begin{tabular}{|c|c|c|c|c|c|}
\hline \multirow[b]{2}{*}{$\lambda / \AA$} & \multirow[b]{2}{*}{ SNR } & \multicolumn{2}{|r|}{ Even } & \multicolumn{2}{|r|}{ Odd } \\
\hline & & J-value & Level Energy $/ \mathrm{cm}^{-1}$ & J-value & Level Energy $/ \mathrm{cm}^{-1}$ \\
\hline 9425.497 & 8 & $11 / 2$ & 14760.320 & $11 / 2$ & 25366.965 \\
\hline 9426.870 & 8 & $3 / 2$ & 11911.437 & $1 / 2$ & $22516.490^{a}$ \\
\hline 9428.620 & 9 & $9 / 2$ & 13605.68 & $9 / 2$ & 24208.775 \\
\hline 9446.839 & 8 & $5 / 2$ & 12481.682 & $7 / 2$ & $23064.370^{a}$ \\
\hline 9471.121 & 15 & $7 / 2$ & 12321.925 & $9 / 2$ & $22877.524^{a}$ \\
\hline 9493.715 & nl 18 & $9 / 2$ & 11184.410 & $11 / 2$ & $21714.802^{a}$ \\
\hline 9503.761 & nl 6 & $13 / 2$ & 10423.680 & $11 / 2$ & $20942.933^{a}$ \\
\hline 9511.74 & 16 & $9 / 2$ & 13974.730 & $9 / 2$ & $24485.187^{a}$ \\
\hline 9553.105 & nl 10 & $3 / 2$ & 12051.5302 & $1 / 2$ & $22516.490^{a}$ \\
\hline 9560.301 & nl 24 & $9 / 2$ & 10356.710 & $9 / 2$ & 20813.780 \\
\hline 9567.360 & 13 & $11 / 2$ & 14760.320 & $11 / 2$ & 25209.670 \\
\hline 9583.331 & 66 & $11 / 2$ & 11282.870 & $11 / 2$ & $21714.802^{a}$ \\
\hline 9586.286 & 14 & $5 / 2$ & 12647.027 & $5 / 2$ & 23075.728 \\
\hline 9651.909 & 15 & $9 / 2$ & 12519.718 & $9 / 2$ & $22877.524^{a}$ \\
\hline 9663.357 & 26 & $5 / 2$ & 9710.640 & $5 / 2$ & 20056.197 \\
\hline 9684.477 & 6 & $15 / 2$ & 20007.142 & $13 / 2$ & 9684.19 \\
\hline 9699.662 & nl 7 & $11 / 2$ & 14178.380 & $9 / 2$ & $24485.187^{a}$ \\
\hline 9716.396 & nl 3 & $9 / 2$ & 11549.614 & $9 / 2$ & 21838.683 \\
\hline 9716.816 & 23 & $9 / 2$ & 5822.905 & $7 / 2$ & 16111.549 \\
\hline 9751.899 & 45 & $1 / 2$ & 12264.888 & $1 / 2$ & $22516.490^{a}$ \\
\hline 9768.643 & 12 & $9 / 2$ & 13974.73 & $9 / 2$ & 24208.775 \\
\hline 9779.645 & $\mathrm{nl} 5$ & $5 / 2$ & 12078.583 & $7 / 2$ & $22301.145^{a}$ \\
\hline 9792.495 & 19 & $5 / 2$ & 11107.705 & $7 / 2$ & 21316.810 \\
\hline 9834.801 & 33 & $9 / 2$ & 11549.614 & $11 / 2$ & $21714.802^{a}$ \\
\hline 9945.496 & nl 8 & $3 / 2$ & 12464.356 & $1 / 2$ & $22516.490^{a}$ \\
\hline 9956.092 & 15 & $15 / 2$ & 16650.620 & $17 / 2$ & $26692.000^{a}$ \\
\hline 9958.534 & 15 & $11 / 2$ & 10904.070 & $11 / 2$ & $20942.933^{a}$ \\
\hline 9864.788 & 18 & $5 / 2$ & 10828.988 & $7 / 2$ & 20963.372 \\
\hline 9878.011 & nl 3 & $13 / 2$ & 12041.668 & $15 / 2$ & 22162.41 \\
\hline
\end{tabular}

$\mathrm{nl}$... new line, ${ }^{a}$ previously unknown engergy level

Table 2: Spectral lines of Pr I with an intensity larger than 50 which clearly could be assigned to a transition between two energy levels

\begin{tabular}{|c|c|c|c|c|c|}
\hline \multirow[b]{2}{*}{$\lambda / \AA$} & \multirow[b]{2}{*}{ SNR } & \multicolumn{2}{|r|}{ Even } & \multicolumn{2}{|r|}{ Odd } \\
\hline & & J-value & Level Energy $/ \mathrm{cm}^{-1}$ & J-value & Level Energy $/ \mathrm{cm}^{-1}$ \\
\hline 3363.938 & nl 53 & $11 / 2$ & 29718.524 & $9 / 2$ & 0 \\
\hline 4216.768 & nl 53 & $11 / 2$ & 4866.530 & $13 / 2$ & 28574.696 \\
\hline 4519.626 & nl 112 & $15 / 2$ & 8363.916 & $15 / 2$ & 30483.514 \\
\hline 4598.951 & nl 178 & $11 / 2$ & 24584.745 & $13 / 2$ & 2846.744 \\
\hline 4692.652 & 55 & $11 / 2$ & 4866.530 & $9 / 2$ & 26170.480 \\
\hline 4795.241 & 50 & $7 / 2$ & 30427.864 & $9 / 2$ & 9579.832 \\
\hline 4802.883 & 55 & $11 / 2$ & 6313.239 & $11 / 2$ & 27128.251 \\
\hline 4825.813 & 70 & $9 / 2$ & 4432.240 & $7 / 2$ & 25148.347 \\
\hline
\end{tabular}

$\mathrm{nl}$... new line, ${ }^{a}$ previously unknown energy level 
Table 2 - Continued

\begin{tabular}{|c|c|c|c|c|c|}
\hline \multirow[b]{2}{*}{$\lambda / \AA$} & \multirow[b]{2}{*}{ SNR } & \multicolumn{2}{|r|}{ Even } & \multicolumn{2}{|r|}{ Odd } \\
\hline & & J-value & Level Energy $/ \mathrm{cm}^{-1}$ & J-value & Level Energy $/ \mathrm{cm}^{-1}$ \\
\hline 4861.964 & 105 & $11 / 2$ & 4866.530 & $11 / 2$ & 25428.607 \\
\hline 4869.332 & 70 & $11 / 2$ & 6892.949 & $13 / 2$ & 27423.911 \\
\hline 4878.194 & nl 97 & $13 / 2$ & 6603.606 & $11 / 2$ & 27097.270 \\
\hline 4911.460 & 50 & $13 / 2$ & 6603.606 & $11 / 2$ & 26958.467 \\
\hline 4915.407 & 230 & $11 / 2$ & 23185.260 & $13 / 2$ & 2846.744 \\
\hline 4916.999 & 90 & $11 / 2$ & 6313.239 & $13 / 2$ & 26645.170 \\
\hline 4925.315 & 450 & $15 / 2$ & 23144.347 & $13 / 2$ & 2846.744 \\
\hline 4927.059 & 55 & $15 / 2$ & 8765.556 & $13 / 2$ & 29055.974 \\
\hline 4961.027 & 290 & $11 / 2$ & 22998.237 & $13 / 2$ & 2846.744 \\
\hline 4964.181 & 55 & $11 / 2$ & 29718.524 & $9 / 2$ & 9579.832 \\
\hline 5017.880 & 65 & $13 / 2$ & 7951.338 & $15 / 2$ & $27879 / 216$ \\
\hline 5018.268 & nl 116 & $13 / 2$ & 31918.637 & $11 / 2$ & 11997.050 \\
\hline 5031.770 & 85 & $11 / 2$ & 9675.040 & $13 / 2$ & 29543.242 \\
\hline 5034.639 & 55 & $15 / 2$ & 8765.556 & $15 / 2$ & 28622.414 \\
\hline 5041.550 & 60 & $13 / 2$ & 7951.338 & $11 / 2$ & 27780.979 \\
\hline 5043.611 & 50 & $7 / 2$ & 7617.455 & $9 / 2$ & 27438.992 \\
\hline 5055.723 & 60 & $9 / 2$ & 8029.290 & $7 / 2$ & 27803.341 \\
\hline 5056.569 & 125 & $11 / 2$ & 4866.530 & $9 / 2$ & 24637.265 \\
\hline 5074.000 & 170 & $11 / 2$ & 4866.530 & $9 / 2$ & 24569.356 \\
\hline 5075.586 & 135 & $11 / 2$ & 8829.090 & $13 / 2$ & 28525.569 \\
\hline 5075.865 & 270 & $9 / 2$ & 5822.905 & $9 / 2$ & 25518.489 \\
\hline 5079.266 & 280 & $11 / 2$ & 22529.130 & $13 / 2$ & 2846.744 \\
\hline 5096.794 & 60 & $13 / 2$ & 6603.606 & $11 / 2$ & 26218.316 \\
\hline 5099.136 & 60 & $9 / 2$ & 5822.905 & $11 / 2$ & 25428.607 \\
\hline 5109.078 & nl 60 & $7 / 2$ & 6535.530 & $5 / 2$ & 26103.032 \\
\hline 5114.261 & 50 & $9 / 2$ & 8643.839 & $7 / 2$ & 28191.557 \\
\hline 5117.291 & 380 & $15 / 2$ & 9745.420 & $17 / 2$ & 29281.511 \\
\hline 5136.270 & 55 & $11 / 2$ & 30793.720 & $9 / 2$ & 11329.710 \\
\hline 5138.297 & 85 & $11 / 2$ & 6714.199 & $9 / 2$ & 26170.480 \\
\hline 5153.249 & 50 & $9 / 2$ & 8643.830 & $9 / 2$ & 28043.749 \\
\hline 5167.541 & 50 & $9 / 2$ & 8643.830 & $11 / 2$ & 27990.060 \\
\hline 5174.777 & 110 & $11 / 2$ & 6313.239 & $11 / 2$ & 25632.322 \\
\hline 5183.491 & 100 & $9 / 2$ & 19286.645 & $9 / 2$ & 0 \\
\hline 5190.494 & 120 & $13 / 2$ & 7951.350 & $15 / 2$ & 27212.011 \\
\hline 5210.956 & 130 & $15 / 2$ & 8765.570 & $15 / 2$ & 27950.542 \\
\hline 5213.404 & 100 & $11 / 2$ & 9675.040 & $13 / 2$ & 28851.059 \\
\hline 5223.986 & 75 & $9 / 2$ & 8643.830 & $11 / 2$ & 27780.979 \\
\hline 5231.691 & 115 & $15 / 2$ & 8765.570 & $15 / 2$ & 27874.491 \\
\hline 5252.448 & 90 & $5 / 2$ & 6451.823 & $7 / 2$ & 25485.269 \\
\hline 5257.536 & 135 & $13 / 2$ & 7630.147 & $13 / 2$ & 26645.170 \\
\hline 5259.274 & 135 & $7 / 2$ & 19008.740 & $9 / 2$ & 0 \\
\hline 5267.660 & 200 & $17 / 2$ & 10532.001 & $19 / 2$ & 29510.473 \\
\hline 5285.628 & 1650 & $13 / 2$ & 20290.580 & $11 / 2$ & 1376.605 \\
\hline 5287.547 & 85 & $7 / 2$ & 6535.587 & $9 / 2$ & 25442.695 \\
\hline 5290.536 & 120 & $11 / 2$ & 6313.239 & $11 / 2$ & 25209.670 \\
\hline 5290.946 & 52 & $13 / 2$ & 6603.606 & $15 / 2$ & 25498.559 \\
\hline 5292.283 & nl 208 & $9 / 2$ & 18890.180 & $9 / 2$ & 0 \\
\hline
\end{tabular}

$\mathrm{nl}$... new line, ${ }^{a}$ previously unknown energy level 
Table 2 - Continued

\begin{tabular}{|c|c|c|c|c|c|}
\hline \multirow[b]{2}{*}{$\lambda / \AA$} & \multirow[b]{2}{*}{ SNR } & \multicolumn{2}{|r|}{ Even } & \multicolumn{2}{|r|}{ Odd } \\
\hline & & J-value & Level Energy $/ \mathrm{cm}^{-1}$ & J-value & Level Energy $/ \mathrm{cm}^{-1}$ \\
\hline 5299.787 & 100 & $7 / 2$ & 6535.587 & $7 / 2$ & 25399.020 \\
\hline 5308.816 & 160 & $13 / 2$ & 6603.606 & $13 / 2$ & 25434.959 \\
\hline 5315.359 & 88 & $13 / 2$ & 10470.300 & $15 / 2$ & 29278.521 \\
\hline 5317.648 & 85 & $11 / 2$ & 8829.078 & $13 / 2$ & 27629.152 \\
\hline 5328.078 & nl 70 & $15 / 2$ & 23144.347 & $15 / 2$ & 4381.100 \\
\hline 5337.453 & nl 51 & $9 / 2$ & 4432.240 & $7 / 2$ & 23162.497 \\
\hline 5341.548 & 60 & $15 / 2$ & 10466.730 & $15 / 2$ & 29182.662 \\
\hline 5341.990 & 70 & $11 / 2$ & 6714.199 & $11 / 2$ & 25428.607 \\
\hline 5342.064 & 66 & $7 / 2$ & 8013.104 & $7 / 2$ & 26727.245 \\
\hline 5370.335 & 145 & $11 / 2$ & 6313.239 & $13 / 2$ & 24928.881 \\
\hline 5376.774 & 66 & $9 / 2$ & 8643.839 & $9 / 2$ & 27237.190 \\
\hline 5391.580 & 58 & $15 / 2$ & 9745.391 & $15 / 2$ & 28287.674 \\
\hline 5394.051 & 91 & $9 / 2$ & 8643.839 & $9 / 2$ & 27177.628 \\
\hline 5424.089 & 64 & $11 / 2$ & 9268.741 & $11 / 2$ & 27699.892 \\
\hline 5435.134 & 55 & $5 / 2$ & 9710.615 & $7 / 2$ & 28104.309 \\
\hline 5445.407 & 89 & $19 / 2$ & 11151.448 & $19 / 2$ & 29510.445 \\
\hline 5453.232 & 118 & $11 / 2$ & 27066.095 & $13 / 2$ & 8733.450 \\
\hline 5456.397 & 69 & $9 / 2$ & 8029.290 & $9 / 2$ & 26351.314 \\
\hline 5456.876 & 56 & $17 / 2$ & 12736.636 & $19 / 2$ & 31057.048 \\
\hline 5462.883 & 61 & $9 / 2$ & 18300.341 & $9 / 2$ & 0 \\
\hline 5469.506 & 189 & $13 / 2$ & 19654.728 & $11 / 2$ & 1376.605 \\
\hline 5471.965 & 79 & $15 / 2$ & 8765.556 & $15 / 2$ & 27035.451 \\
\hline 5514.111 & 75 & $9 / 2$ & 8029.290 & $11 / 2$ & 26159.548 \\
\hline 5514.574 & 55 & 4.5 & 4432.24 & 4.5 & $22560.965^{a}$ \\
\hline 5530.631 & 53 & $15 / 2$ & 8765.556 & $15 / 2$ & 26841.658 \\
\hline 5534.181 & 55 & $7 / 2$ & 8013.104 & $7 / 2$ & 26077.608 \\
\hline 5538.374 & nl 240 & $11 / 2$ & 26784.270 & $13 / 2$ & 8733.450 \\
\hline 5590.462 & 60 & $11 / 2$ & 4866.530 & $11 / 2$ & $22749.175^{a}$ \\
\hline 5594.760 & 82 & $9 / 2$ & 4432.240 & $7 / 2$ & $22301.160^{a}$ \\
\hline 5617.656 & 54 & $17 / 2$ & 11714.367 & $19 / 2$ & 29510.445 \\
\hline 5624.964 & 213 & $7 / 2$ & 6535.587 & $9 / 2$ & 24308.545 \\
\hline 5634.928 & 80 & $9 / 2$ & 10356.710 & $11 / 2$ & 28098.267 \\
\hline 5645.683 & 64 & $15 / 2$ & 22088.820 & $15 / 2$ & 4381.100 \\
\hline 5649.278 & 56 & $11 / 2$ & 9268.741 & $13 / 2$ & 26965.207 \\
\hline 5720.451 & 88 & $7 / 2$ & 28412.960 & $9 / 2$ & 10936.670 \\
\hline 5728.378 & 70 & $17 / 2$ & 14028.722 & $19 / 2$ & 31480.828 \\
\hline 5746.622 & 54 & $19 / 2$ & 29309.852 & $19 / 2$ & 11913.160 \\
\hline 5748.007 & 75 & $13 / 2$ & 6603.606 & $15 / 2$ & 23996.130 \\
\hline 5753.327 & 61 & $11 / 2$ & 9268.741 & $13 / 2$ & 26645.170 \\
\hline 5756.391 & nl 81 & $11 / 2$ & 8829.078 & $13 / 2$ & 26196.253 \\
\hline 5843.479 & 53 & $9 / 2$ & 8320.255 & $11 / 2$ & 25428.607 \\
\hline 5893.492 & 234 & $11 / 2$ & 6714.199 & $13 / 2$ & 23677.365 \\
\hline 5907.249 & 112 & $9 / 2$ & 18300.341 & $11 / 2$ & 1376.605 \\
\hline 5929.330 & 80 & $11 / 2$ & 18237.240 & $11 / 2$ & 1376.605 \\
\hline 5936.246 & nl 58 & $9 / 2$ & 8029.290 & $11 / 2$ & 24870.318 \\
\hline 5949.765 & nl 171 & $17 / 2$ & 27523.120 & $17 / 2$ & 10720.400 \\
\hline 5960.933 & 110 & $7 / 2$ & 7617.455 & $5 / 2$ & $24388.702^{a}$ \\
\hline
\end{tabular}

$\mathrm{nl}$... new line, ${ }^{a}$ previously unknown energy level 
Table 2 - Continued

\begin{tabular}{|c|c|c|c|c|c|}
\hline \multirow[b]{2}{*}{$\lambda / \AA$} & \multirow[b]{2}{*}{ SNR } & \multicolumn{2}{|r|}{ Even } & \multicolumn{2}{|r|}{ Odd } \\
\hline & & J-value & Level Energy $/ \mathrm{cm}^{-1}$ & J-value & Level Energy $/ \mathrm{cm}^{-1}$ \\
\hline 5987.819 & nl 94 & $9 / 2$ & 8320.255 & $11 / 2$ & 25016.201 \\
\hline 5994.418 & nl 82 & $9 / 2$ & 18054.202 & $11 / 2$ & 1376.605 \\
\hline 6023.642 & 68 & $15 / 2$ & 27265.622 & $15 / 2$ & 10668.960 \\
\hline 6030.234 & 54 & $15 / 2$ & 28342.772 & $17 / 2$ & 11764.250 \\
\hline 6061.992 & 70 & $9 / 2$ & 27145.754 & $7 / 2$ & 10654.110 \\
\hline 6074.993 & 134 & $9 / 2$ & 24706.499 & $9 / 2$ & 8250.170 \\
\hline 6083.735 & 78 & $11 / 2$ & 6714.199 & $13 / 2$ & 23146.917 \\
\hline 6085.810 & 142 & $9 / 2$ & 24677.280 & $9 / 2$ & 8250.170 \\
\hline 6107.014 & 90 & $9 / 2$ & 25205.463 & $11 / 2$ & 8835.380 \\
\hline 6118.016 & 50 & $11 / 2$ & 24590.800 & $9 / 2$ & 8250.170 \\
\hline 6236.807 & 102 & $19 / 2$ & 29309.852 & $17 / 2$ & 13280.440 \\
\hline 6238.372 & 95 & $7 / 2$ & 6535.587 & $9 / 2$ & $22560.965^{a}$ \\
\hline 6280.999 & 54 & $11 / 2$ & 27662.982 & $13 / 2$ & 11746.340 \\
\hline 6440.364 & 53 & $13 / 2$ & 13146.599 & $11 / 2$ & 28669.174 \\
\hline 6440.366 & 54 & $15 / 2$ & $26191.740^{a}$ & $15 / 2$ & 10668.960 \\
\hline 6441.818 & 56 & $15 / 2$ & 27265.622 & $13 / 2$ & 11746.340 \\
\hline 6486.560 & 650 & $17 / 2$ & 25059.089 & $15 / 2$ & 9646.850 \\
\hline $6539 / 224$ & 61 & $19 / 2$ & 30098.998 & $19 / 2$ & 14799.880 \\
\hline 6616.025 & 196 & $7 / 2$ & 15110.661 & $9 / 2$ & 0 \\
\hline 6617.336 & 51 & $15 / 2$ & $24759 / 250$ & $15 / 2$ & 9646.850 \\
\hline 6725.360 & 85 & $5 / 2$ & 6451.823 & $7 / 2$ & 21316.810 \\
\hline 6749.209 & 50 & $15 / 2$ & 8765.556 & $15 / 2$ & $23578.018^{a}$ \\
\hline 6845.305 & 58 & $5 / 2$ & 6451.823 & $5 / 2$ & $21056.348^{a}$ \\
\hline 6931.411 & 65 & $11 / 2$ & 6313.239 & $9 / 2$ & $20736.338^{a}$ \\
\hline 6995.508 & 52 & $7 / 2$ & 6535.587 & $5 / 2$ & $20826.530^{a}$ \\
\hline 7083.429 & 200 & $13 / 2$ & 9464.460 & $15 / 2$ & $23578.018^{a}$ \\
\hline 7255.175 & 380 & $13 / 2$ & 15156.070 & $11 / 2$ & 1376.605 \\
\hline 7278.726 & 110 & $11 / 2$ & 15111.480 & $11 / 2$ & 1376.605 \\
\hline 7889.311 & 60 & $17 / 2$ & 9770.330 & $15 / 2$ & $22442.185^{a}$ \\
\hline 8395.932 & 65 & $11 / 2$ & 8829.078 & $9 / 2$ & $20736.338^{a}$ \\
\hline 8986.243 & 95 & $19 / 2$ & 13626.720 & $19 / 2$ & $24751.780^{a}$ \\
\hline 8993.509 & 67 & $9 / 2$ & 19196.472 & $11 / 2$ & 8080.440 \\
\hline 9144.025 & 53 & $5 / 2$ & 9710.615 & $3 / 2$ & 20643.800 \\
\hline 9293.861 & 50 & $5 / 2$ & 9710.640 & $5 / 2$ & $20467.450^{a}$ \\
\hline 9319.006 & 50 & $17 / 2$ & 11714.367 & $15 / 2$ & $22442.185^{a}$ \\
\hline 9583.331 & 66 & $11 / 2$ & 11282.870 & $11 / 2$ & $21714.802^{a}$ \\
\hline
\end{tabular}

nl ... new line, ${ }^{a}$ previously unknown engergy level 
Table 3. Spectral lines of Pr II which clearly could be assigned to a transition between two energy levels

\begin{tabular}{|c|c|c|c|c|c|}
\hline \multirow[b]{2}{*}{$\lambda / \AA$} & \multirow[b]{2}{*}{ SNR } & \multicolumn{2}{|r|}{ Even } & \multicolumn{2}{|r|}{ Odd } \\
\hline & & J-value & Level Energy $/ \mathrm{cm}^{-1}$ & J-value & Level Energy $/ \mathrm{cm}^{-1}$ \\
\hline 3898.837 & nl 11 & 7 & 14035.46 & 8 & 39676.86 \\
\hline 3956.753 & nl 49 & 6 & 5854.54 & 6 & 31120.63 \\
\hline 3974.851 & nl 9 & 9 & 13639.13 & 9 & 38790.18 \\
\hline 4010.602 & nl 14 & 7 & 11253.46 & 7 & 36180.33 \\
\hline 4014.331 & nl 3 & 6 & 10137.93 & 7 & 35041.68 \\
\hline 4022.712 & nl 10 & 8 & 13021.33 & 8 & 37873.15 \\
\hline 4062.220 & nl 6 & 8 & 12237.15 & 8 & 36847.30 \\
\hline 4087.209 & $\mathrm{nl} 5$ & 9 & 14330.52 & 9 & 38790.18 \\
\hline 4133.612 & nl 11 & 6 & 9211.95 & 7 & 33397.06 \\
\hline 4176.318 & nl 7 & 6 & 11283.14 & 5 & 35220.93 \\
\hline 4213.554 & nl 8 & 6 & 9670.82 & 7 & 33397.06 \\
\hline 4223.466 & nl 6 & 6 & 10454.26 & 5 & 34124.85 \\
\hline 4306.037 & nl 9 & 5 & 9733.44 & 6 & 32950.10 \\
\hline 4382.390 & nl 6 & 6 & 10137.93 & 6 & 32950.10 \\
\hline 4394.984 & nl 6 & 7 & 10650.24 & 7 & 33397.06 \\
\hline 4446.986 & nl 5 & 5 & 8379.46 & 6 & 30860.28 \\
\hline 5201.579 & nl 15 & 4 & 14167.90 & 5 & 33387.64 \\
\hline 5219.048 & 38 & 7 & 25569.19 & 7 & 6413.93 \\
\hline 5567.521 & nl 2 & 8 & 13021.33 & 7 & 30977.67 \\
\hline
\end{tabular}


Table 4: New levels found by analysis of hf patterns in the FT spectrum.

\begin{tabular}{|c|c|c|c|c|c|c|}
\hline energy $/ \mathrm{cm}^{-1}$ & $\mathrm{~J}$-value & $\mathrm{A}^{1} / \mathrm{MHz}$ & parity & \multicolumn{3}{|c|}{ lines which can be explained $(\AA)$} \\
\hline $19002.837(5)$ & $7 / 2$ & $804(2)$ & o & $9069.905,8018.809,79$ & 5.293 & \\
\hline $20467.450(10)$ & $5 / 2$ & $792(16)$ & o & $9293.861,9241.771,80$ & 7.119 & \\
\hline $20736.338(5)$ & $9 / 2$ & $611(3)$ & o & $\begin{array}{l}9062.401, \quad 8717.830, \\
7620.501, \quad 7221.674, \\
6703.511,6299.531\end{array}$ & $\begin{array}{l}\text { 8395.932, } \\
7039.939\end{array}$ & $\begin{array}{l}7857.830 \\
6931.411\end{array}$ \\
\hline $20826.530(10)$ & $5 / 2$ & $822(4)$ & o & $\begin{array}{l}9403.206 \quad 9164.799, \\
7568.4686995 .502,695\end{array}$ & $\begin{array}{l}8944.852 \\
1.743\end{array}$ & 7802.166 \\
\hline 20942.933(5) & $11 / 2$ & $363(3)$ & o & $\begin{array}{l}9958.534, \quad 9503.761, \\
7509.564,7115.484,70\end{array}$ & $\begin{array}{l}8724.067 \\
6.094\end{array}$ & 8709.564 \\
\hline $21056.348(5)$ & $5 / 2$ & 1378(3) & $\mathrm{o}$ & $\begin{array}{l}9204.274, \quad 8975.694, \\
7439.040,6845.305\end{array}$ & 8811.465 & 8754.630 \\
\hline $21714.802(5)$ & $11 / 2$ & $1100(3)$ & o & $\begin{array}{l}9834.801, \quad 9583.331, \\
8160.791,7097.968,67\end{array}$ & $\begin{array}{l}9493.715 \\
4.932\end{array}$ & 8801.914, \\
\hline $22301.145(5)$ & $7 / 2$ & $746(3)$ & o & $\begin{array}{ll}9779.645, & 9298.448, \\
8257.844, & 7320.071, \\
5594.760 & \end{array}$ & $\begin{array}{l}9232.162 \\
7150.649\end{array}$ & $\begin{array}{l}9066.240, \\
6808.396,\end{array}$ \\
\hline $22442.185(5)$ & $15 / 2$ & $959(9)$ & o & $\begin{array}{l}9612.268, \quad 9319.006, \\
8210.847, \quad 7889.311, \\
7101.187,6311.952\end{array}$ & $\begin{array}{l}9189.156 \\
7873.840\end{array}$ & $\begin{array}{l}\text { 8393.847, } \\
7309.733,\end{array}$ \\
\hline $22516.49(5)$ & $1 / 2$ & $-994(5)$ & o & $\begin{array}{l}9945.496, \quad 9751.939, \\
9385.756,8926.403\end{array}$ & 9553.105 & 9426.870 \\
\hline $22560.965(5)$ & $9 / 2$ & $920(5)$ & o & $\begin{array}{l}9079.042, \quad 9015.841 \\
6153.001,5514.574\end{array}$ & 7280.314 & 6283.372 , \\
\hline $22749.175(5)$ & $11 / 2$ & $878(9)$ & o & $\begin{array}{l}9336.680, \quad 8718.817 \\
6191.936,5590.462\end{array}$ & 8111.028, & 7536.197 \\
\hline $22877.524(5)$ & $9 / 2$ & $931(5)$ & o & $\begin{array}{l}9651.909, \quad 9471.121, \\
8825.333, \quad 8615.883, \\
7714.329, \quad 7463.978, \\
6035.413,5419.933\end{array}$ & $\begin{array}{l}9143.853 \\
8549.691 \\
7346.172\end{array}$ & $\begin{array}{l}8954.679, \\
8349.501, \\
6117.532,\end{array}$ \\
\hline $22964.630(5)$ & $7 / 2$ & 1109(3) & o & $\begin{array}{l}7542.806, \quad 7539.478, \\
6054.231\end{array}$ & 6826.625 & 6686.434, \\
\hline $23064.370(10)$ & $7 / 2$ & $749(3)$ & o & $\begin{array}{l}9076.121, \quad 8682.117, \\
7483.183\end{array}$ & 8624.300 & 8415.219 \\
\hline $23353.116(5)$ & $7 / 2$ & $858(5)$ & $\mathrm{o}$ & $\begin{array}{l}9337.905, \quad 8705.521, \\
8276.642, \quad 7328.016, \\
6650.257,6523.982\end{array}$ & $\begin{array}{l}8539.710 \\
7240.324\end{array}$ & $\begin{array}{r}8588.806, \\
7016.555\end{array}$ \\
\hline $23578.018(5)$ & $15 / 2$ & $535(5)$ & o & $7599.958,7083.429,67$ & $9.209^{2}, 6571$ & $.034^{2}$ \\
\hline $24183.415(10)$ & $11 / 2$ & $969(3)$ & o & $9058.096,8233.786,72$ & $5.574,6630$. & 181 \\
\hline $24289.760(10)$ & $19 / 2$ & $990(3)$ & o & $8653,316,7609.254^{2}, 6$ & 85.420 & \\
\hline $24388.702(5)$ & $5 / 2$ & 1114(3) & $\mathrm{o}$ & $\begin{array}{l}9110.821, \quad 5810.994, \\
5599.709,5573.558\end{array}$ & 6782.978 & 5960.933 \\
\hline $24485.187(5)$ & $9 / 2$ & $1110(2)$ & o & $\begin{array}{l}9699.662, \quad 9511.740, \\
6069.197,5569.608\end{array}$ & 9176.122 & 8453.074, \\
\hline $24751.780(10)$ & $19 / 2$ & 1012(2) & o & $8986.241,8320.561,76$ & 8.138 & \\
\hline
\end{tabular}

${ }^{1}$ The value of the hyperfine constant B could not be determined with significance therefore it is not taken into account in this work. B was set to zero when determining $\mathrm{A}$.

2 The line was already classified in ref. [4], but this classification is wrong. 
Table 4 - Continued

\begin{tabular}{ccccl}
\hline energy $/ \mathrm{cm}^{-1}$ & J-value & $\mathrm{A}^{1} / \mathrm{MHz}$ & parity & lines which can be explained $(\AA)$ \\
\hline $26191.740(10)$ & $15 / 2$ & $478(3)$ & e & $6920.705,6440.366,6334.990,5726.350$ \\
$26692.000(5)$ & $17 / 2$ & $837(5)$ & o & $9956.092, \quad 9216.883^{2}, \quad 8069.405^{2}, \quad 7651.767$, \\
& & & & 7163,745
\end{tabular}

${ }^{1}$ The value of the hyperfine constant $\mathrm{B}$ could not be determined with significance therefore it is not taken into account in this work. B was set to zero when determining A.

${ }^{2}$ The line was already classified in reference [4], but this classification is wrong.

\section{Conclusion}

In this work, we have concentrated on investigations of the hyperfine structure of the Pr atom and ion based on new FT spectra. With the help of these FT spectra we could enlarge the wavelength list of Pr from around 17000 up to 26000 lines. Furthermore we assigned more than 1200 lines to a transition between two known energy levels. By fitting several lines in the IR region we were also able to determine 24 new atomic levels. Even though this number is small compared to the approximately 2000 known energy levels, it shows the potential of FT spectra in classifying lines and finding new energy levels. The new FT spectra of Pr I and Pr II will be very helpful for further laser induced fluorescence spectroscopy experiments because now one can determine exactly possible excitation wavelengths for an investigation of promising structures. The full list of all more than 26000 lines is available on request. 


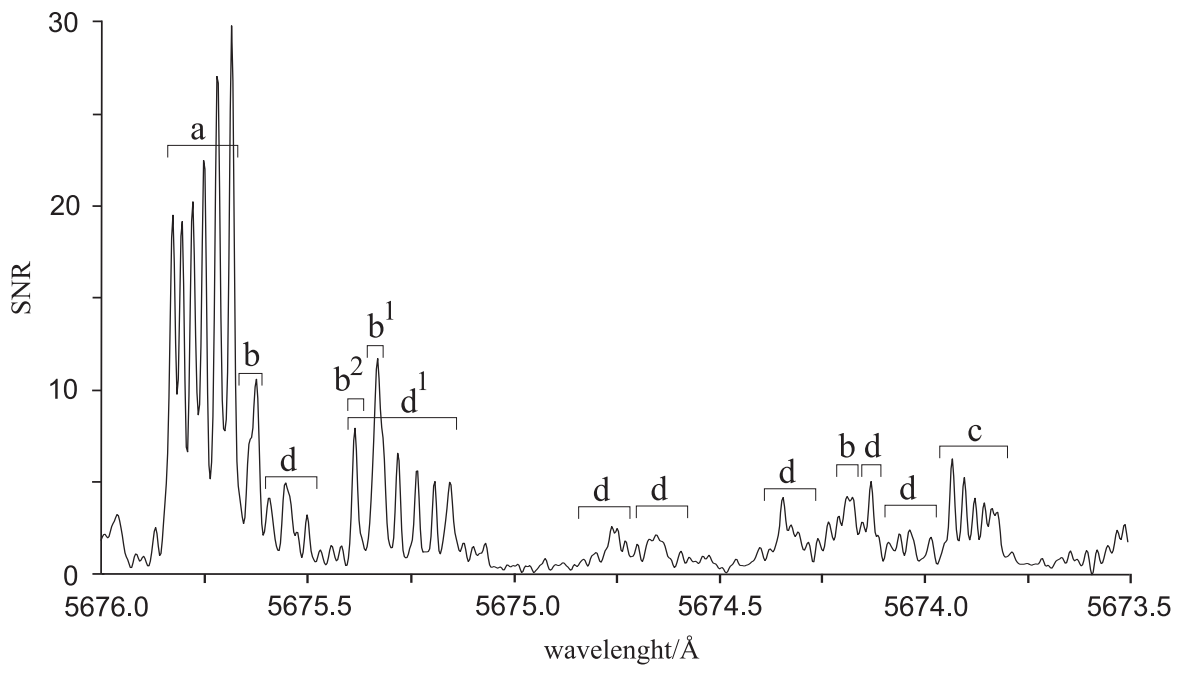

Figure 1. Part of the FT spectrum in the visible region.

a ... line classified by Ginibre (ref. [4])

b ... line given in the tables of Ginibre (ref. [4]), but not classified

c ... line classified before having the FT spectrum by laser spectroscopy (ref. [9])

d ... new line, discovered with help of this spectrum

Around $5675.75 \AA$ a typical blend situation can be noticed: a spectral line with a wide $\mathrm{hf}$ splitting $\left(\mathrm{d}^{1}\right)$ is overlapped by another line with a small hf splitting $\left(\mathrm{b}^{1}\right)$. The most intense component of the widely splitted line was noticed by Ginibre (ref. [4] and treated to be a spectral line with small hf splitting $\left(\mathrm{b}^{2}\right)$.

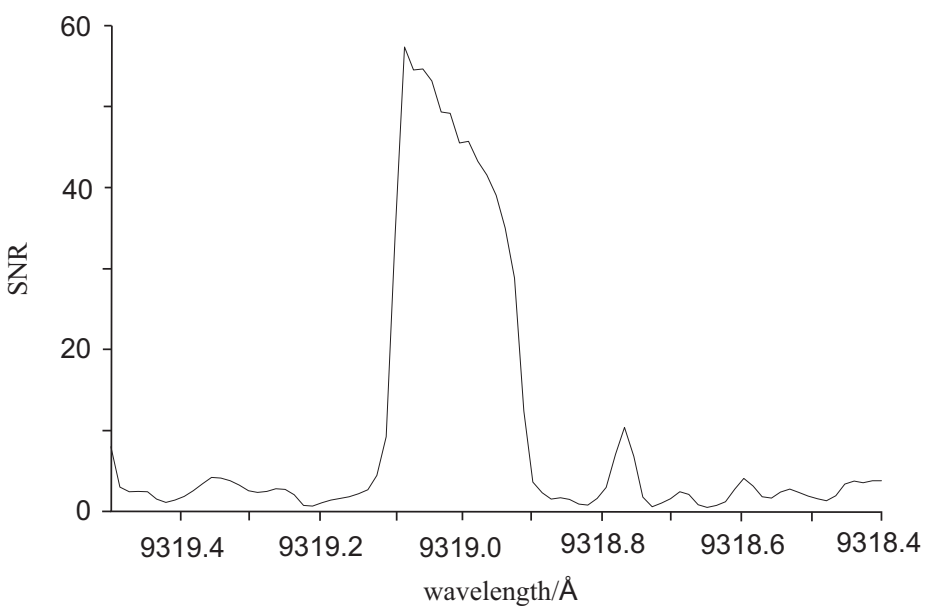

Figure 2. Appearance of the line $9319.006 \AA$ in the FT spectrum 

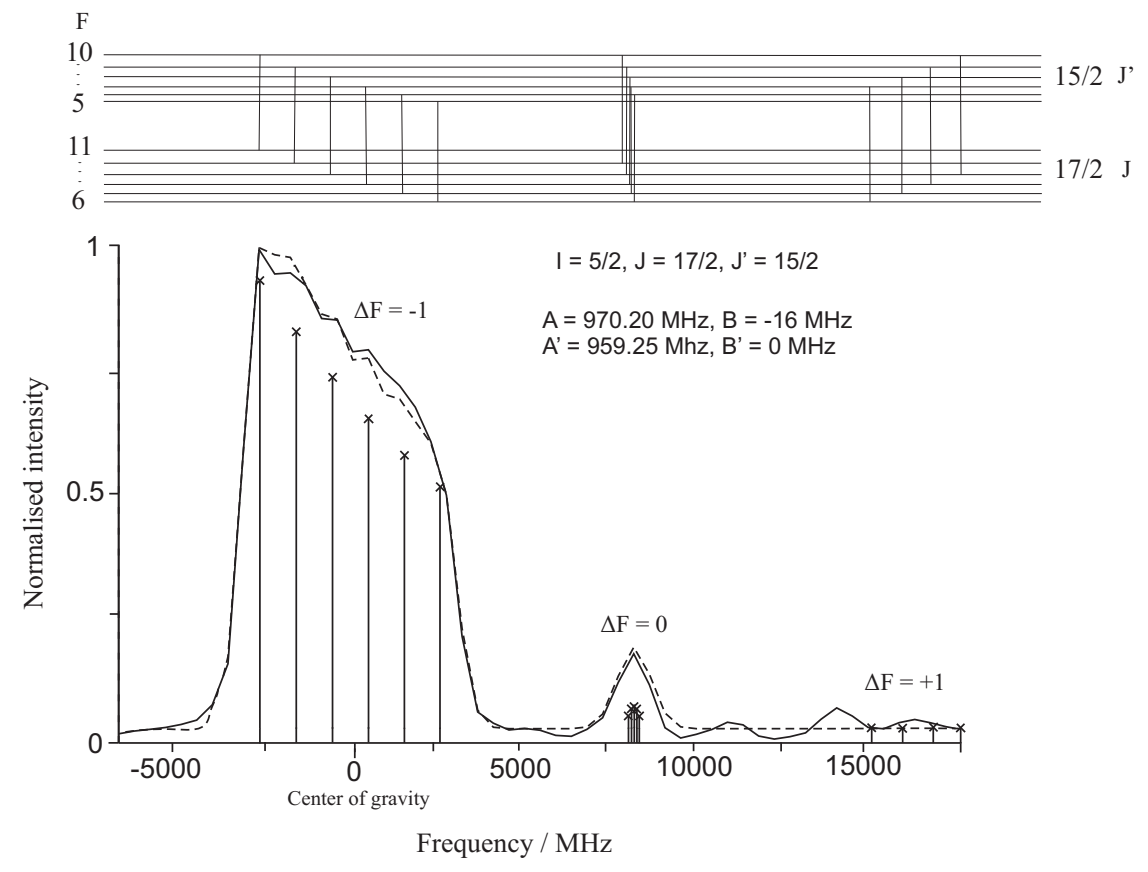

Figure 3. Best fit of the FT spectrum of the line $9319.006 \AA$ assuming a transition $J=17 / 2$ to $J^{\prime}=15 / 2$. The hf constants of the lower level were fixed, the B factor of the upper level was assumed to be zero. The appearance of the line in the FT spectra (solid line) is compared to the fitted line profile (dashed line). All spectral lines appear in the FT spectrum with relative intensity ratios which are very close to the theoretical ones. The upper part of the figure shows the hf level scheme and all transitions. 


\section{References}

[1] King A 1928 Astrophys. J. 68194

[2] Ginibre A 1989 Phys. Scripta 39694

[3] Ginibre A 1989 Phys. Scripta 39710

[4] Ginibre A 1988 PhD Thesis Paris

[5] Ruczkowski J, Stachowska E, Elantkowska M, Guthöhrlein G H and Dembczyński J 2003 Phys. Scripta 68133

[6] Furmann B, Stefańska D, Dembczyński J and Stachowska E 2005 Phys. Scripta 72300

[7] Furmann B, Stefańska D, Dembczyński J and Stachowska E 2006 Phys. Scripta 74658

[8] Guthöhrlein G H, Helmut Schmidt-Universität der Bundeswehr (Holstenhofweg 85, D-22043 Hamburg, Germany), private communication

[9] Uddin Z PhD Thesis Graz 2006 unpublished

[10] Gamper B Diploma Thesis Graz 2007 unpublished

[11] Jaritz N, Jäger H and Windholz L 2002 Eur. Phys. J. D 18267

[12] Peck R and Reeder K 1972 J. Opt. Soc. Am. 62958

[13] Windholz L and Guthöhrlein G H 2003 Phys. Scripta T105 55

[14] Learner R C M and Thorne A P 1988 J. Opt. Soc. Am. B 52045

[15] Messnarz D, Jaritz N, Arcimowicz B, Zilio V O, Engleman, Jr. R, Pickering J C, Jäger H, Guthöhrlein G H and Windholz L 2003 Phys. Scripta 68170

[16] Martin W C, Zalubas R, Hagan L. 1978. Atomic Energy Levels - The Rare Earth Elements. Natl. Bur. Stand., NSRDS-NBS 60, p.98, U.S. G.P.O. Washington D.C.

[17] Program package "Fitter", developed by Guthöhrlein G H, Institute for Experimental Physics, Helmuth Schmidt Universität der Bundeswehr Hamburg 1998 